\title{
OPTIMAL MULTIPHASE TRANSPORTATION WITH PRESCRIBED MOMENTUM
}

\author{
YANn Brenier ${ }^{1}$ AND MARJolaine PUeL ${ }^{2}$
}

\begin{abstract}
A multiphase generalization of the Monge-Kantorovich optimal transportation problem is addressed. Existence of optimal solutions is established. The optimality equations are related to classical Electrodynamics.
\end{abstract}

Mathematics Subject Classification. 65K10, 35Q.

Received January 14, 2002.

\section{INTRODUCTION}

\section{Optimal Transportation Theory}

The origin of optimal transportation problems goes back to the memoir "sur les déblais et les remblais" published by Monge near 1780. A new formulation of these problems in terms of infinite dimensional linear programs was introduced by Kantorovich around 1940 leading to the so-called Monge-Kantorovich problem (see the book by Rachev and Rüschendorf [23] for a detailed review):

Given two probability measures $\rho_{0}, \rho_{1}$ on a metric space $D$, find a probability measure $\mu$ on the product space $D \times D$, with projections $\rho_{0}$ and $\rho_{1}$ on each copy of $D$, that minimizes the cost

$$
\int_{D^{2}} \operatorname{cost}(x, y) \mathrm{d} \mu(x, y)
$$

where $\operatorname{cost}(x, y)$ is the "transportation cost" to go from point $x \in D$ to point $y \in D$, usually defined in terms of the distance $\mathrm{d}(x, y)$ between $x$ and $y$. In this formulation, $\mu(x, y)$ should be understood as the probability for point $x \in D$ to be transported at point $y \in D$. In the special case when

$$
\operatorname{cost}(x, y)=\frac{\mathrm{d}(x, y)^{p}}{p}
$$

(where $1 \leq p<+\infty$ ) the optimal cost raised to the power $1 / p$ defines a distance on the $\operatorname{set} \operatorname{Prob}(D)$ of all (Borel) probability measures on $D$. This distance, called Monge-Kantorovich distance (or Wasserstein distance, there are many other names) with exponent $p$, confers a metric structure to the weak-* topology of Prob $(D)$.

Keywords and phrases: Optimal transportation, multiphase flow, electrodynamics.

1 Laboratoire J.A Dieudonné, Parc Valrose, 06100 Nice, France; e-mail: brenier]math.unice.fr

2 Laboratoire d'Analyse Numérique, Université Paris 6, BC. 187, 75252 Paris Cedex 05, France. 
In the case when $D$ is a Riemannian manifold and $p=2$, the Monge-Kantorovich distance confers a (formal) Riemannian structure to $\operatorname{Prob}(D)$, as shown by Otto [20].

The discrete counterpart of the Monge-Kantorovich problem belongs to the field of Operational Research and Combinatorial Optimization (Linear Assignment Problems, in particular, see [24] for an elementary introduction).

A bridge between Optimal Transportation Theory $(O T T)$ and non-linear PDEs was established by the first author in [6] (see [7] for more details) where the quadratic OTT corresponding to exponent $p=2$ and $D=\mathbb{R}^{d}$ was shown to be directly related to the (real) Monge-Ampère equation (a fully non-linear elliptic PDE with strong geometric features [22]). Earlier and more involved connexions with non-linear PDEs were established by Cullen and Purser in [13], where a variant of the quadratic OTT was involved in the definition of the semigeostrophic model for atmospheric fronts, and in [5] (which motivated [6]) where the quadratic OTT was derived from a time discretization of the Euler equations of incompressible fluids written in Lagrangian coordinates. Once the regularity analysis of the quadratic $O T T$ in connexion with the Monge-Ampère equation was achieved by Caffarelli [12], OTT has became a flourishing field of non-linear PDEs. It has been discovered since that many other important non-linear PDEs have an underlying optimal transportation structure. In particular the original Monge problem (which corresponds to exponent $p=1$ ), studied by Sudakov [25] in the Kantorovich setting, was related to the Eikonal equations by Evans and Gangbo [14] and can be applied to Optimum Design, as shown by Bouchitté and Buttazzo [4]. A large collection of dissipative equations, such as the heat equation, the so-called porous media equation, the thin film equations, some granular flow equations, etc. have been also interpreted as gradient flows for the Monge-Kantorovich (formally Riemannian) metric corresponding to exponent $p=2[16,19,20]$ etc.

Optimal Transportation Theory also turns out to be a powerful tool in theoretical Statistics, Functional Analysis and Calculus of Variations $[1,18,21], \ldots$

A different formulation of the quadratic $O T T$ in terms of classical Continuum Mechanics was proposed by Benamou and the first author in [2] for numerical purposes:

Given a compact convex domain $D$ in $\mathbb{R}^{d}$, and two probability measures $\rho_{0}$ and $\rho_{1}$ on $D$, find a nonnegative time and space dependent measure $c(t, x)$, for $t \in[0,1]$ and $x \in D$ and a $c$ - square integrable vector field $v(t, x) \in \mathbb{R}^{d}$, parallel to the boundary $\partial D$, minimizing

$$
\int \frac{1}{2}|v(t, x)|^{2} c(\mathrm{~d} t, \mathrm{~d} x)
$$

subject to $\partial_{t} c+\operatorname{div}(c v)=0$ and to initial and final conditions

$$
c(t=0, x)=\rho_{0}(x), \quad c(t=1, x)=\rho_{1}(x) .
$$

(We have used notations $|$.$| for the Euclidean norm, div =\nabla$. for the divergence operator, where $\nabla=$ $\left(\partial_{x_{1}}, \ldots, \partial_{x_{d}}\right)$ and $\cdot$ is the Euclidean inner product in $\mathbb{R}^{d}$.)

It was shown that the resulting infimum coincide with the optimal transportation cost corresponding to $\operatorname{cost}(x, y)=1 / 2|x-y|^{2}$ (see [10] for a detailed proof). Notice that this minimization problem is no longer a linear program involving a nonnegative measure $\mu$ defined on the $2 d$ dimensional product space $D \times D$, as the Monge-Kantorovich problem is, but rather a convex (homogeneous of degree 1) minimization problem in the nonnegative and vector-valued measures $c$ and $m=c v$ defined on the $d+1$ dimensional product space $[0,1] \times D$. This alternative formulation has a very simple interpretation in terms of Fluid Mechanics. It amounts to minimize the kinetic energy of a fluid carrying the density field from $\rho_{0}$ at time $t=0$ to $\rho_{1}$ at time $t=1$. The optimality condition (at least at the formal level) for the fluid to achieve the infimum is extremely simple. The velocity field must be potential and the acceleration field must be null

$$
v=\nabla \phi, \quad \partial_{t} v+(v \cdot \nabla) v=0 .
$$

(See [2] for more details.) 


\section{Multiphase Optimal Transportation theory}

The Fluid Mechanics formulation of the Optimal Transportation problem has many possible extensions. One possible extension is the optimal transportation of currents, instead of densities, as discussed in [10], which provides non linear models for classical Electrodynamics related to the Born-Infeld theory [3]. The second possible extension is the optimal transportation of multiple phases in a domain $D$ and in a time interval $[0, T]$. Imagine that instead of a single phase, described as above by its density and velocity fields $c(t, x), v(t, x)$, depending on time $t \in[0, T]$ and space $x \in D$, we rather consider several phases, labeled by $a$, each of them having its own density and velocity fields $c(t, x, a), v(t, x, a)$, still subject to

$$
\partial_{t} c+\operatorname{div}_{x}(c v)=0
$$

$v$ being tangent to the boundary $\partial D$. Then, we can define the total density

$$
\rho(t, x)=\sum_{a} c(t, x, a)
$$

It is natural to prescribe $\rho(t, x)=1$, which means that all the volume available is occupied by the different phases. (Then $c(t, x, a)$ can also be seen as the concentration of phase $a$ at time $t$ and point $x$.) A natural cost is the kinetic energy of the phases integrated in both $t \in[0, T]$ and $a$ and weighted by $w(a) \geq 0$,

$$
\sum_{a} \int_{0}^{T} \int_{D} \frac{1}{2} w(a)|v(t, x, a)|^{2} c(t, x, a) \mathrm{d} t \mathrm{~d} x .
$$

Then, given the concentration fields at time $t=0$ and time $t=T$, namely $c_{0}(x, a)$ and $c_{T}(x, a)$, we may look for an optimal transportation plan $(c, v)$. This is still a convex (homogeneous of degree 1) minimization problem in the concentration fields $c(t, x, a)$ and momentum fields $m(t, x, a)=v(t, x, a) c(t, x, a)$. There are many possible interpretations of this multiphase optimal transportation problem with prescribed total density. For instance, it could correspond to a continuous version of the following rather realistic network problem for traffic flows (cars, airplanes, computers,...):

Several groups of individuals (each group being labeled by a) must collectively move from their initial location at time $t=0$ distributed in space according to the concentration field $c_{0}(x, a)$ (each individual being indistinguishable from the other members of its own group) to their final destination distributed according to $c_{T}(x, a)$ at time $T$, through a given network $D$. During their motion, the different groups are subject to share all the available room at each time $t$ and each point $x$ of the network $D$. A particularly relevant situation is the case when a "void" phase is introduced, say with label $a=0$ and $w(0)=0$. Then for the other phases, the constraint

$$
\sum_{a \neq 0} c(t, x, a) \leq 1
$$

says that there is a saturation rate in the network not to be exceeded, and the total cost to be minimized is just

$$
\sum_{a \neq 0} \int \frac{1}{2} w(a)|v(t, x, a)|^{2} c(t, x, a) \mathrm{d} t \mathrm{~d} x .
$$

The multiphase optimal transportation problem with density constraint has been first addressed in [8] in the special case of equal weights $w(a)=1$ and related to the Euler equations of incompressible fluids. A more refined analysis (including the case when the label variable $a$ belongs to a measure space $(A, d a)$ which need not be discrete) was provided in [9]. It is shown that, in the case $D=[0,1]^{d}, w(a)=1$, there are always optimal 
plans $c(t, x, a), v(t, x, a)$ that are solutions (in a suitable weak sense) of the following set of PDEs:

$$
\begin{gathered}
\partial_{t} c+\operatorname{div}_{x}(c v)=0, \quad \sum_{a} c(t, x, a)=1, \\
\partial_{t} v+\left(v \cdot \nabla_{x}\right) v+\nabla p=0
\end{gathered}
$$

where $p(t, x)$, the Lagrange multiplier associated with the density constraint, does not depend on the label variable $a$. The later equation implies that all phases $a$ have the same acceleration field (namely $\nabla p$ ) which does not depend on $a$. In addition $\nabla p$ is shown to be uniquely determined by the data $c_{0}(x, a)$ and $c_{T}(x, a)$. In other words, in the framework of networks, there is a neutral way of driving particles which does not depend on the phase they belong to. This property of the multiphase optimal transportation problem might be useful for some tentative applications. (For instance, in air traffic control, as explained to us by Delahaye, it is important to use rules that do not discriminate among air companies. In our case, the acceleration field does not discriminate among phases.)

In the present paper, a similar multiphase optimal transportation problem is addressed where the cost function is unchanged, but the constraint is no longer on the total density field $\rho(t, x)=\sum_{a} c(t, x, a)$ but rather on the total momentum $u(t, x)$, defined by

$$
u(t, x)=\sum_{a} v(t, x, a) c(t, x, a) .
$$

So, we assume that $u$ is prescribed. Notice that $\rho$ and $u$ are linked by the continuity equation

$$
\partial_{t} \rho+\operatorname{div} u=0 .
$$

Thus, if we assume the initial and final values of $c(t, x, a)$ to satisfy the compatibility condition

$$
\sum_{a} c_{0}(x, a)=\sum_{a} c_{T}(x, a)=1
$$

and $u$ to be divergence free, then automatically $\rho=1$ will be enforced. So, under these assumptions, we can see the total momentum constraint as a reinforcement of the total density constraint previously considered. (Except in the very special case of one space dimension $d=1$, when both constraints are merely equivalent.)

As a first "application" of the multiphase optimal transportation problem, we may use the momentum constraint just to get a suboptimal solution of the problem with density constraint. For instance, if there are only two phases, $a=0$ and $a=1$, and the total momentum is prescribed to be null, $u=0$, then we can reduce the optimal two-phase transportation problem to a single phase transportation problem with a modified cost. Indeed, it is enough to set

$$
m(t, x)=m(t, x, 0)=-m(t, x, 1), \quad c(t, x)=c(t, x, 0)=1-c(t, x, 1),
$$

to see that the two-phase problem is equivalent to

$$
\inf \int_{0}^{T} \int_{D} \frac{1}{2}|m(t, x)|^{2}\left(\frac{w(0)}{c(t, x)}+\frac{w(1)}{1-c(t, x)}\right) \mathrm{d} t \mathrm{~d} x
$$

subject to $\partial_{t} c+\operatorname{div} m=0$, with $c(t, x)$ prescribed at $t=0$ and $t=T$ and valued in $[0,1]$. Such a reduction to a single-phase problem would not be possible with the density constraint (except in the very special case of one space dimension $d=1$ ).

More generally, various applications of the "multiphase optimal transportation problem with prescribed total momentum" to networks and traffic flow are conceivable. Let us just quote an example. In some situations, 
it might be desirable to move individuals from their initial position to their final position with zero total momentum $u=0$. Such a motion, for instance, would be invisible to any observer that is only able to measure total density and total momentum. (Of course one of the phases could be used as a lure for this furtive motion.)

\section{Optimality equations}

Let us now briefly discuss what are the expected optimality equations of the multiphase optimal transportation problem with prescribed total momentum. To get them, let us first introduce two Lagrange multipliers, the first one for the continuity equation of each phase, denoted by $\phi(t, x, a) \in \mathbb{R}$, and the second one, denoted by $H(t, x) \in \mathbb{R}^{d}$, depending only on the time and space variables, to enforce the total momentum to be pointwise equal to $u(t, x)$. So, we get the following saddle-point problem

$$
\begin{aligned}
\inf _{c, v} \sup _{H, \phi} \sum \int & \left\{\frac{1}{2} w(a)|v(t, x, a)|^{2}-\partial_{t} \phi(t, x, a)-\left(H(t, x)+\nabla_{x} \phi(t, x, a)\right) \cdot v(t, x, a)\right\} c(t, x, a) \mathrm{d} t \mathrm{~d} x \\
& +\int H(t, x) \cdot u(t, x) \mathrm{d} t \mathrm{~d} x+\int\left(\phi(1, x, a) c_{T}(x, a)-\phi(0, x, a) c_{0}(x, a)\right) \mathrm{d} x
\end{aligned}
$$

(Indeed, the supremum in $H$ and $\phi$ is either 0 or $+\infty$ depending on whether or not the constraints are fulfilled.) The formal optimality equations can be obtained by varying this expression with respect to $c$ and $v$. (Notice that it would be more correct to use $c$ and $m=c v$ instead of $c$ and $v$ since the optimal transportation problem is convex in $(c, m)$ not in $(c, v)$. However, the formal optimality equations do not depend on such a change of variable.) Let us derive these equations in the most interesting case $d=3$. We get, first by varying $v$,

$$
w(a) v(t, x, a)=H(t, x)+\nabla_{x} \phi(t, x, a),
$$

next by varying $c$,

$$
\frac{1}{2} w(a)|v(t, x, a)|^{2}-\partial_{t} \phi(t, x, a)-\left(H(t, x)+\nabla_{x} \phi(t, x, a)\right) \cdot v(t, x, a)=0
$$

We can eliminate the Lagrange multiplier $\phi$ by taking the curl of the first equation and the gradient of the second one, which leads to:

$$
\begin{gathered}
w(a) \operatorname{curl} v(t, x, a)=\operatorname{curl} H(t, x) \\
\partial_{t}(w(a) v(t, x, a)-H(t, x))+\nabla_{x}\left(\frac{1}{2} w(a)|v(t, x, a)|^{2}\right)=0 .
\end{gathered}
$$

We can write the second equation as

$$
w(a)\left(\partial_{t}+v(t, x, a) . \nabla_{x}\right) v(t, x, a)=E(t, x)+v(t, x, a) \wedge B(t, x),
$$

where

$$
E(t, x)=\partial_{t} H(t, x), \quad B(t, x)=-\operatorname{curl} H(t, x),
$$

satisfy

$$
\operatorname{div} B=0, \quad \partial_{t} B+\operatorname{curl} E=0 .
$$


Thus, each phase $a$ is driven by the same "Lorentz force" generated by the fields $E$ and $B$ which do not depend on labels $a$. An equivalent formulation is

$$
w(a)\left(\partial_{t}(c v)+\operatorname{div}_{x}(c v \otimes v)\right)(t, x, a)=c(t, x, a)(E(t, x)+v(t, x, a) \wedge B(t, x)) .
$$

\section{A link with Classical Electrodynamics}

In the special case when all the weights are equal, say $w(a)=1$, and constraints $u=0, \rho=1$ are enforced, we can write $(2)$ in terms of the "phase density" $f(t, x, \xi)$ defined by

$$
f(t, x, \xi)=\sum_{a} \delta(\xi-v(t, x, a)) c(t, x, a), \quad \xi \in \mathbb{R}^{3}
$$

The constraints become

$$
\int_{\mathbb{R}^{3}} f(t, x, \mathrm{~d} \xi)=1, \quad \int_{\mathbb{R}^{3}} \xi f(t, x, \mathrm{~d} \xi)=0
$$

and the dynamical equation reads

$$
\partial_{t} f+\operatorname{div}_{x}(\xi f)+\operatorname{div}_{\xi}((E+\xi \wedge B) f)=0 .
$$

So we have obtained a system of equations which is very close to the Vlasov equations of Classical Electrodynamics describing the motion of electrons moving in a uniform background of unit positive charge. The Vlasov equations involve the "phase density" $f(t, x, \xi) \geq 0$ of the electrons and the electromagnetic field $(E, B)$. They include equation (1) and the "relativistic version" of (3), namely

$$
\partial_{t} f+\operatorname{div}_{x}(\tilde{\xi} f)+\operatorname{div}_{\xi}((E+\tilde{\xi} \wedge B) f)=0,
$$

where $\tilde{\xi}=C \xi / \sqrt{\xi^{2}+C^{2}}, C$ is the speed of light and the physical units are chosen so that electrons have unit mass and charge. However, in the Vlasov equations, there are no density and momentum constraints. Instead, the inhomogeneous part of the Maxwell equations is used, namely

$$
\epsilon_{0} \operatorname{div} E(t, x)=1-\int_{\mathbb{R}^{3}} f(t, x, \mathrm{~d} \xi), \quad \epsilon_{0}\left(\partial_{t} E(t, x)-C^{2} \operatorname{curl} B(t, x)\right)=\int_{\mathbb{R}^{3}} \tilde{\xi} f(t, x, \mathrm{~d} \xi),
$$

where $\epsilon_{0}$ is the electric constant. However, at any physical scale where $\epsilon_{0} C^{2}$ and $C^{-1}$ are negligible, these equations can be seen as a relaxation of the total density and momentum constraints. Conversely, the equations of the multiphase optimal transportation problem with prescribed total momentum can be seen (at least in the case of equal weights $w(a)=1$ and constraint $u=0$ ) as a (singular) limit of the Classical Electrodynamics equations.

\section{Main mathematical results}

We will restrict ourself to the particular case when the domain $D$ is the periodic cube $D=\mathbb{R}^{3} / \mathbb{Z}^{3}$, the phases are equally weighted $w(a)=1$, and the total density and the total momentum are prescribed to be respectively 1 and 0 . Then, it will be shown, for general (compatible) initial and final data $c_{0}(x, a), c_{T}(x, a)$ in the class of (Borel) probability measures on $D \times A$, that the multiphase optimal transportation problem always admit a solution $(c, v, E, B)$, where $c(t, x, a)$ is a nonnegative measure, $v(t, x, a)$ is square integrable in $(t, x, a)$ with respect to $c, E(t, x) \in \mathbb{R}^{3}$ is a locally bounded measure and $B(t, x)$ is square integrable with respect to the Lebesgue measure. In addition, optimality equations (2) are satisfied in a suitable weak sense. 


\section{Definitions, Assumptions And Results}

\subsection{Mathematical description of a multiphase flow}

We consider a multiphase flow moving, during a fixed time interval $[0, T]$, in the periodic cube $D=\mathbb{R}^{d} / \mathbb{Z}^{d}$ with $d=3$. (General notation $d$ will be kept in all proofs where the operator curl is not involved.) Each phase is labeled by $a \in A$, where $A$ is a (topological) probability space $(A, d a)$, typically $A=[0,1]$ or $A=D$, equipped with the Lebesgue measure. (Indeed, our proofs do no require $a$ to be a discrete variable.) The motion of each phase $a$ is described by a density field $c(t, x, a) \geq 0$ and a velocity field $v(t, x, a) \in \mathbb{R}^{d}$, where $t \in[0, T]$ and $x \in D$. Mathematically, $c$ will be considered as a nonnegative (Borel) measure on the product space $Q^{\prime}=Q \times A$ where $Q=[0, T] \times D$, and $v$ as a vector-valued function on $Q^{\prime}$, square integrable with respect to $c$, so that the "Action" of the multiphase flow

$$
\int_{Q^{\prime}} \frac{1}{2}|v(t, x, a)|^{2} \mathrm{~d} c(t, x, a)
$$

(where all phases are equally weighted by $w(a)=1$ ) is finite. A useful formulation of the Action can be obtained by introducing the "momentum" field defined by $m(t, x, a)=v(t, x, a) c(t, x, a)$. This makes $m$ a vector-valued measures on $Q^{\prime}$, absolutely continuous with respect to $c, v$ being the Radon-Nikodym density of $m$ with respect to $c$. Let us introduce, for all pair $(c, m)$ of (Borel) measures on $Q^{\prime}$ respectively valued in $\mathbb{R}$ and $\mathbb{R}^{d}$, the convex function valued in $[0,+\infty]$ and defined by

$$
K(c, m)=\sup _{F, \Phi} \int_{Q^{\prime}} F(t, x, a) \mathrm{d} c(t, x, a)+\Phi(t, x, a) \cdot \mathrm{d} m(t, x, a)
$$

where $F$ and $\Phi$ are all continuous functions on $Q^{\prime}$ respectively valued in $\mathbb{R}$ and $\mathbb{R}^{d}$ subject to

$$
F(t, x, a)+\frac{1}{2}|\Phi(t, x, a)|^{2} \leq 0 \text { pointwise. }
$$

It is known (see [8], for instance) that $K(c, m)$ is finite if and only if $c$ is nonnegative, $m$ is absolutely continuous with respect to $c$ and can be written $m=v(t, x, a) c(t, x, a)$ with $v \in L^{2}\left(Q^{\prime}, \mathrm{d} c ; \mathbb{R}^{d}\right)$, in which case

$$
K(c, m)=\int_{Q^{\prime}} \frac{1}{2}|v(t, x, a)|^{2} \mathrm{~d} c(t, x, a) .
$$

Thus $K(c, m)$ provides a nice definition of the Action as a convex function of the pair $(c, m)$. We further restrict ourself to pairs $(c, m)$ that satisfy the continuity equation

$$
\partial_{t} c+\operatorname{div}_{x} m=0
$$

and initial and final conditions

$$
\left.c(0, x, a)=c_{0}(x, a), \text { and } c(T, x, a)=c_{T}(x, a)\right),
$$

in the following weak sense

$$
\int_{Q^{\prime}}\left(\partial_{t} f(t, x, a) \mathrm{d} c(t, x, a)+\nabla_{x} f(t, x, a) \cdot \mathrm{d} m(t, x, a)\right)=\int f(T, x, a) c_{T}(\mathrm{~d} x, \mathrm{~d} a)-f(0, x, a) c_{0}(\mathrm{~d} x, \mathrm{~d} a)
$$

for all functions $f$ continuous on $Q^{\prime}$, with continuous first order derivatives in $(t, x)$. Here $c_{0}$ and $c_{T}$ are given probability measures on $D \times A$. The total density and momentum are (weakly) prescribed by assuming

$$
\int_{Q^{\prime}} f(t, x) \mathrm{d} c(t, x, a)=\int_{Q} f(t, x) \mathrm{d} t \mathrm{~d} x
$$


for all continuous function $f$ on $Q$, and

$$
\int_{Q^{\prime}} H(t, x) \cdot \mathrm{d} m(t, x, a)=\int_{Q} H(t, x) \cdot u(t, x) \mathrm{d} t \mathrm{~d} x
$$

for all continuous $H$ defined on $Q$ and valued in $\mathbb{R}^{d}$. Here $u$ is a given smooth divergence free vector field on $Q$ subject to further restrictions. Notice that these conditions are compatible precisely because $u$ is divergence free and the continuity equation is enforced. All these conditions imply that $c(t, x, a)$ (and similarly $m(t, x, a)$ ) can be desintegrated as a probability measure on $A$, Lebesgue measurable in $(t, x) \in Q$. Because of the continuity equation, $c$ can also be seen as a probability measure on $D \times A$, continuously depending on $t \in$ $[0, T]$. Therefore, notations $\mathrm{d} c(t, x, a)=c(\mathrm{~d} t, \mathrm{~d} x, \mathrm{~d} a), c(t, x, \mathrm{~d} a), c(t, \mathrm{~d} x, \mathrm{~d} a), \mathrm{d} m(t, x, a)=v(t, x, a) \mathrm{d} c(t, x, a)$, $m(t, x, \mathrm{~d} a)=v(t, x, a) c(t, x, \mathrm{~d} a)$, will be used according to the context. The total density constraint can be expressed by

$$
\int c(t, x, \mathrm{~d} a)=1
$$

for all $t$. The data $c_{0}$ and $c_{T}$ are accordingly required to satisfy

$$
\int c_{0}(x, \mathrm{~d} a)=\int c_{T}(x, \mathrm{~d} a)=1
$$

Then, condition (9) automatically follows from (7) and (8). We further assume $c_{0}$ and $c_{T}$ to satisfy

$$
\int c_{0}(\mathrm{~d} x, a)=\int c_{T}(\mathrm{~d} x, a)=1
$$

so that, for each $(t, a), c(t, x, a)$ is a probability measure in $x$. Finally, notice that the Action can be written as the time integral of the "kinetic" energy defined by

$$
\int_{Q} \frac{1}{2}|v(t, x, a)|^{2} c(t, \mathrm{~d} x, \mathrm{~d} a)
$$

for all $t \in[0, T]$.

\subsection{The optimal transportation problem}

We are now looking for a pair $(c, m)$ minimizing $K(c, m)$ and subject to constraints $(7,8)$. This amounts to solve the following saddle-point problem

$$
I\left(c_{0}, c_{T}\right)=\inf _{(c, m) \in M} \sup _{(H, \phi) \in \mathcal{H}} L(c, m, \phi, H)
$$

where $L$ denotes

$$
\begin{aligned}
L(c, m, \phi, H)= & K(c, m)-\int_{Q^{\prime}} \partial_{t} \phi(t, x, a) \mathrm{d} c(t, x, a)-\int_{Q^{\prime}}\left[\nabla_{x} \phi(t, x, a)+H(t, x)\right] \cdot \mathrm{d} m(t, x, a) \\
& +\int \phi(T, x, a) c_{T}(\mathrm{~d} x, \mathrm{~d} a)-\phi(0, x, a) c_{0}(\mathrm{~d} x, \mathrm{~d} a)+\int_{Q} H(t, x) \cdot u(t, x) \mathrm{d} t \mathrm{~d} x
\end{aligned}
$$

with $M=\mathcal{M} \times \mathcal{M}^{d}$, where $\mathcal{M}$ is the set of all (Borel) measures (or, equivalently by Riesz' theorem, the set of all linear continuous forms on $\left.C\left(Q^{\prime}\right)\right)$, and $\mathcal{H}=C\left(Q ; \mathbb{R}^{d}\right) \times C^{1,1,0}\left(Q^{\prime}\right)$, where $C^{1,1,0}\left(Q^{\prime}\right)$ stands for the space 
of all continuous real-valued function $f(t, x, a)$ of $(t, x, a) \in Q^{\prime}$ with continuous partial derivatives with respect to $t$ and $x$.

So far, the prescribed total momentum $u(t, x)$ is only assumed to be smooth and divergence free. Our main result is only proven in the case when $u=0$. However, most of the intermediary results require less restrictive conditions.

\subsection{The main result}

Theorem 1.1. 1) Assume $D=\mathbb{T}^{d}=\mathbb{R}^{d} / \mathbb{Z}^{d}, c_{0}(x, a) \geq 0$ and $c_{T}(x, a) \geq 0$ are probability measures in $(x, a) \in D \times A$ subject to

$$
\begin{aligned}
& \int c_{0}(x, \mathrm{~d} a)=\int c_{T}(x, \mathrm{~d} a)=1, \\
& \int c_{0}(\mathrm{~d} x, a)=\int c_{T}(\mathrm{~d} x, a)=1 .
\end{aligned}
$$

Let $u(t, x)$ be a smooth divergence field. Then, there is always at least a solution $(c, m)$ of the optimal multiphase transportation problem. Any optimal solution $(c, m)$ satisfies

$$
\begin{gathered}
m=c v \text { and } \partial_{t} c+\operatorname{div}_{x} m=0, \\
\int m(t, x, \mathrm{~d} a)=u(t, x) \text { and } \int c(t, x, \mathrm{~d} a)=1, \\
K(c, m) \leq C
\end{gathered}
$$

where $C=C(T, d, u)$ does not depend on initial and final conditions.

2) In addition, if $u=0$, then $C(T, d, u) \leq d^{2} / T$, the kinetic energy $\frac{1}{2} \int|v|^{2} c(t, \mathrm{~d} x, \mathrm{~d} a)$ is time independent and there is a vector field $H(t, x)$ such that for all optimal $(c, m)$

$$
\partial_{t}(c v)+\operatorname{div}_{x}(c v \otimes v)-\underline{c} \partial_{t} H+c v \wedge \operatorname{curl} H=0 .
$$

In this equation, $E=\partial_{t} H$ is a locally bounded measure in the interior of $Q, B=-\operatorname{curl} H$ is square integrable in $Q$ with respect to the Lebesgue measure and $\underline{c}(t, x, a)$ is a well defined extension of $c(t, x, a)$ to the singular set of $E$ with respect to the Lebesgue measure.

Remark 1.1. A precise definition of $\underline{c}$ is as follows. Since $E=\partial_{t} H$ is a locally bounded vector-valued measure on the interior of $Q$, we may consider $|E|$ as a nonnegative Borel measure on $Q$ and consider the Banach space $L^{1}(Q,|E| ; C(A))$ of all (strongly) $|E|$ measurable and integrable functions $f$ on $Q$ valued in the Banach space $C(A)$ of all continuous functions on $A$, for which

$$
\left\||| f\left|\|=\int_{t, x}\right| E(t, x)\left|\sup _{a \in A}\right| f(t, x, a) \mid<\infty .\right.
$$

It will be shown that, for each fixed $f \in L^{1}(Q,|E| ; C(A))$, the following integral

$$
I_{\delta, \gamma, e}(f)=\int_{t, x} E(t, x) \int_{\mathbb{R}^{d} \times A} f(t, x, a) c(t, \mathrm{~d} z, \mathrm{~d} a) \gamma\left(\frac{x-\delta e-z}{\delta}\right) \frac{1}{\delta^{d}}
$$


(which is well defined for all radial mollifiers $\gamma$ and all unit vector $e$, since $c(t, x, a)$ depends continuously on $t$ as a measure in $(x, a))$ has a unique limit $I(f)$, as $\delta \rightarrow 0$, that do not depend on $\gamma$ or $e$. In addition we have $|I(f)| \leq|||f||| \int|E|$. Thus, $f \rightarrow I(f)$ defines $\underline{c}$ as a continuous linear form on $L^{1}(Q,|E| ; C(A))$ and we can write

$$
I(f)=\int_{t, x} E(t, x) \int_{A} f(t, x, a) \underline{c}(t, x, \mathrm{~d} a) .
$$

As we denote by $E^{r} \in L^{1}\left(Q ; \mathbb{R}^{d}\right)$ the regular part of $E$ with respect to the Lebesgue measure, we get (by standard measure theory)

$$
\int_{Q} E^{r}(t, x) \mathrm{d} t \mathrm{~d} x \int_{\mathbb{R}^{d} \times A} f(t, x, a) c(t, \mathrm{~d} z, \mathrm{~d} a) \gamma\left(\frac{x-\delta e-z}{\delta}\right) \frac{1}{\delta^{d}} \rightarrow \int_{Q} E^{r}(t, x) \mathrm{d} t \mathrm{~d} x \int_{A} f(t, x, a) c(t, x, \mathrm{~d} a) .
$$

Thus, $\underline{c}$ provides an extension of $c$ to the singular set of $E$.

\section{MAIN STEPS OF THE PROOFS}

First, we show the existence of an admissible solution $(c, m)$ with finite Action and obtain

$$
I\left(c_{0}, c_{T}\right) \leq K(c, m) \leq C(T, d, u),
$$

where $C=C(T, d, u)$ depends on $T, d$ and $u$ but not on the initial and final data $c_{0}, c_{T}$. This is enough to enforce that $I\left(c_{0}, c_{T}\right)$ is achieved by at least one optimal pair $(c, m)$.

Next, we use the Fenchel-Rockafellar duality theorem to show the existence of approximate optimal Lagrange multipliers for the constraints:

Proposition 2.1. For each $\varepsilon \geq 0$ there is a pair of continuous functions $\phi_{\varepsilon}(t, x, a)$ on $Q^{\prime}$ and $H_{\varepsilon}(t, x)$ on $Q$ with $\partial_{t} \phi_{\varepsilon}, \nabla_{x} \phi_{\varepsilon}$ continuous on $Q$ ' such that, for all optimal pair $(c, m)$,

$$
\begin{aligned}
& \partial_{t} \phi_{\varepsilon}+\frac{1}{2}\left|\nabla_{x} \phi_{\varepsilon}+H_{\varepsilon}\right|^{2} \leq 0 \\
& \text { and } \\
& \int_{Q^{\prime}}\left(\left|\partial_{t} \phi_{\varepsilon}+\frac{1}{2}\right| \nabla_{x} \phi_{\varepsilon}+\left.H_{\varepsilon}\right|^{2}\left|+\frac{1}{2}\right| v-\nabla_{x} \phi_{\varepsilon}+\left.H_{\varepsilon}\right|^{2}\right) \mathrm{d} c \leq \varepsilon^{2} .
\end{aligned}
$$

Next, we prove some compactness for $H_{\varepsilon}$ or, more precisely, for $E_{\varepsilon}=\partial_{t} H_{\varepsilon}$ and $B_{\varepsilon}=-\operatorname{curl} H_{\varepsilon}$, which define $H_{\varepsilon}$ up to an irrelevant gauge. We use the Sobolev space $G_{m_{0}}=H^{1, m_{0}}([0, T] \times D)$ of all functions of $(t, x) \in[0, T] \times D$ with partial derivatives in $L^{2}$ up to first order in $t$ and up to $m_{0}$-th order in $x$, where $m_{0} \in \mathbb{N}$ has to be chosen large enough.

Proposition 2.2. Assume that $u=0$. Then $\left\langle E_{\varepsilon} ; g\right\rangle,\left\langle B_{\varepsilon} ; g\right\rangle$ are bounded uniformly with respect to $\varepsilon$ for all functions $g(t, x)$ compactly supported in $0<t<T$ with a finite norm in $G$.

Next, we get some estimates that formally mean that the gradient in $x$ of $v(t, x, a)$ (which is not well defined a priori) is in $L^{2}\left(Q^{\prime}, \mathrm{d} c\right)$.

Proposition 2.3. Let $0 \leq \tau \leq \frac{T}{2}$ and $Q_{\tau}^{\prime}=[\tau, T-\tau] \times D \times A$. Let $w(x)$ be a smooth divergence free field on $D$ and $(t, x) \rightarrow \mathrm{e}^{t w}(x)$ be the corresponding flow defined by

$$
\partial_{t}\left(\mathrm{e}^{t w}(x)\right)=w\left(\mathrm{e}^{t w}(x)\right), \quad \mathrm{e}^{(t=0) w}(x)=x .
$$

Then we have

$$
\int_{Q_{\tau}^{\prime}}\left|\left(\nabla_{x} \phi_{\varepsilon}+H_{\varepsilon}\right)(t, x, a)-v(t, x, a)\right|^{2} \mathrm{~d} c(t, x, a) \leq C \varepsilon^{2}
$$




$$
\begin{gathered}
\int_{Q_{\tau}^{\prime}}\left|\left(\nabla_{x} \phi_{\varepsilon}+H_{\varepsilon}\right)(t, x, a)\right|^{2} \mathrm{~d} c(t, x, a) \leq C \\
\int_{Q_{\tau}^{\prime}}\left|\left(\nabla_{x} \phi_{\varepsilon}+H_{\varepsilon}\right)\left(t, \mathrm{e}^{\delta w}(x), a\right)-\left(\nabla_{x} \phi_{\varepsilon}+H_{\varepsilon}\right)(t, x, a)\right|^{2} \mathrm{~d} c(t, x, a) \leq C\left(\varepsilon^{2}+\delta^{2}+\delta \chi(\varepsilon)\right)
\end{gathered}
$$

for all optimal pair $(c, m)$ and for all $\delta$ and $\varepsilon$ small enough, where $\chi(\varepsilon) \geq \varepsilon$ tends to zero with $\varepsilon$. Here $C$ depends only on $D, T, \tau$ and $w$.

These estimates enable us to get a first set of approximate optimality equations.

More precisely:

Proposition 2.4. For all continuous functions $f(t, x, a)$, valued in [0,1], compactly supported in $0<t<T$, with continuous derivatives in $(t, x)$, we have, for all vectors $\omega \in \mathbb{R}^{d}$ and $\delta>0$,

$$
\begin{aligned}
& \mid \int_{Q^{\prime}} \int_{0}^{1}-\partial_{t} H_{\varepsilon}(t, x+\sigma \delta \omega) \cdot \omega f(t, x, a) \mathrm{d} c \mathrm{~d} \sigma+\int_{Q^{\prime}} \int_{0}^{1}\left[v(t, x, a) \wedge \operatorname{curl}\left(H_{\varepsilon}(t, x+\sigma \delta \omega)\right] \cdot \omega f(t, x, a) \mathrm{d} c \mathrm{~d} \sigma\right. \\
& -\int_{Q^{\prime}} \partial_{t} f(t, x, a) v(t, x, a) \cdot \omega \mathrm{d} c-\int_{Q^{\prime}} v(t, x, a) \cdot \nabla_{x} f(t, x, a) v(t, x, a) \cdot \omega \mathrm{d} c \mid \leq C_{f} \frac{1}{\delta}\left(\delta^{2}+\varepsilon^{2}+\delta \chi(\varepsilon)\right) .
\end{aligned}
$$

Next, we get estimates that formally mean that the time derivative of $v(t, x, a)$ (which is not well defined a priori) is in $L^{2}\left(Q^{\prime}, \mathrm{d} c\right)$.

Proposition 2.5. Let $0 \leq \tau \leq \frac{T}{2}$ and $Q_{\tau}^{\prime}=[\tau, T-\tau] \times D \times A$. Then

$$
\int_{Q_{\tau}^{\prime}}\left|v(t, x, a)-\left(\nabla_{x} \phi_{\varepsilon}+H_{\varepsilon}\right)(t+\eta, x, a)\right|^{2} \mathrm{~d} c(t, x, a) \leq C\left(\varepsilon^{2}+\eta^{2}+\eta \chi^{\prime}(\varepsilon)\right)
$$

for all optimal pair $(c, m)$ and for all $\eta$ and $\varepsilon$ small enough, where $\chi^{\prime}(\varepsilon) \geq \varepsilon$ tends to zero with $\varepsilon$. Here $C$ depends only on $D, T, \tau$ and $w$.

Finally, we are able to pass to the limit (through an appropriate balance between the small parameters $\epsilon, \delta$ and $\eta$ ) and get, after several intermediate steps,

Proposition 2.6. $B=-\operatorname{curl} H$ belongs to $L^{2}\left(Q ; \mathbb{R}^{d}\right)$ and $E=\partial_{t} H$ is a locally bounded measure in the interior of $Q$. In addition, $E$ and $B$ solve

$$
\partial_{t}(c v)+\operatorname{div}_{x}(c v \otimes v)-\underline{c} E-c v \wedge B=0 .
$$

\section{Construction of AN ADmissible pair}

Let us check that there is at least an admissible pair $(c, m)$, i.e. satisfying $(7,8)$, with a finite Action, i.e. such that $\int|v|^{2} \mathrm{~d} c<+\infty$.

We closely follow the construction used by the first author in [9]. As a matter of fact, in the special case $u=0$, there is essentially no change to be made. First, as in [9], we define, for $t \in[0, T], x, y, z \in D=\mathbb{R}^{d} / \mathbb{Z}^{d}$, $G(t, x, y, z)$ to be the concatenation of geodesic curves on the periodic box $D$, with constant speed, connecting respectively $x$ to $y$ for $0 \leq t \leq T / 2, y$ to $z$ for $T / 2 \leq t \leq T$ and uniquely defined for almost all pairs $(x, y)$ and $(y, z)$. Next, we introduce the Lebesgue measure-preserving flow $X(t, x)$, associated to $u(t, x)$ through

$$
\begin{aligned}
\partial_{t} X(t, x) & =u(t, X(t, x)) \\
X(0, x) & =x .
\end{aligned}
$$


(In the particular case $u=0$, we just have $X(t, x)=x$.) Let $c_{0}(x, a), c_{T}(x, a)$ be two nonnegative measures defined on $D \times A$ and satisfying

$$
\begin{gathered}
\int c_{0}(x, \mathrm{~d} a)=\int c_{T}(x, \mathrm{~d} a)=1 \\
\int c_{0}(\mathrm{~d} x, a)=\int c_{T}(\mathrm{~d} x, a)=1 .
\end{gathered}
$$

First, we define $c^{0, T}(x, z, a)$ as a measure on $D^{2} \times A$, through the duality bracket

$$
\left\langle c^{0, T} ; f\right\rangle=\int_{D \times A} \int_{D} f(x, z, a) c_{0}(\mathrm{~d} x, a) c_{T}(\mathrm{~d} z, \mathrm{~d} a)
$$

for all continuous functions $f$ on $D^{2} \times A$. (Observe that

$$
\sup _{(z, a)}\left|\int_{D} f(x, z, a) c_{0}(\mathrm{~d} x, a)\right| \leq \sup |f|
$$

since $\int c_{0}(\mathrm{~d} x, a)=1$. Thus, $\int_{D} f(x, z, a) c_{0}(\mathrm{~d} x, a)$ defines a $d c_{T}$ integrable function of $(x, z) \in D \times A$. $)$ Now, we define for every test function $f$,

$$
\begin{aligned}
\langle c ; f\rangle & =\int_{[0, T] \times D^{3} \times A} f(t, X(t, G(t, x, y, z)), a) \mathrm{d} c^{0, T}(x, z, a) \mathrm{d} y \mathrm{~d} t \\
\langle m ; f\rangle & =\int_{[0, T] \times D^{3} \times A} \partial_{t}(X(t, G(t, x, y, z))) f(t, X(t, G(t, x, y, z)), a) \mathrm{d} c^{0, T}(x, z, a) \mathrm{d} y \mathrm{~d} t .
\end{aligned}
$$

This pair satisfies the continuity equation and the boundary conditions in the weak sense of (7). Let us just check (8) in the special case when the total momentum $u$ is zero, in which case $X(t, x)=x$. Then, we have for all continuous function $H(x)$, and for all $0 \leq t \leq T / 2$,

$$
\begin{aligned}
\int H(x) m(t, \mathrm{~d} x, \mathrm{~d} a) & =\int_{D^{3} \times A} \partial_{t} G(t, x, y, z) H(G(t, x, y, z)) \mathrm{d} c^{0, T}(x, z, a) \mathrm{d} y \\
& =\int_{D^{3} \times A} \partial_{t} G(t, x, y, z) H(G(t, x, y, z)) c_{0}(\mathrm{~d} x, a) c_{T}(\mathrm{~d} z, \mathrm{~d} a) \mathrm{d} y
\end{aligned}
$$

(by definition of $c^{0, T}$ ),

$$
=\int_{D^{3} \times A} \partial_{t} \Gamma(t, x, y) H(\Gamma(t, x, y)) c_{0}(\mathrm{~d} x, a) c_{T}(\mathrm{~d} z, \mathrm{~d} a) \mathrm{d} y
$$

(where, by definition of $G$, since for $0 \leq t \leq T / 2, G(t, x, y, z)=\Gamma(t, x, y)$ is a geodesic curve on $D$ with constant speed linking $x$ at $t=0$ to $y$ at $t=T / 2$ )

$$
=\int_{D^{2} \times A} \partial_{t} \Gamma(t, x, y) H(\Gamma(t, x, y)) c_{0}(\mathrm{~d} x, \mathrm{~d} a) \mathrm{d} y
$$

(by integrating out $z \in D$ and using that $\int c_{T}(\mathrm{~d} z, a)=1$ ),

$$
=\int_{D^{2}} \partial_{t} \Gamma(t, x, y) H(\Gamma(t, x, y)) \mathrm{d} x \mathrm{~d} y
$$


(by integrating out $a \in A$, since $\int c_{0}(x, \mathrm{~d} a)=1$ )

$$
=\int_{D^{2}} \partial_{t} \Gamma(t, 0, y-x) H(x+\Gamma(t, 0, y-x)) \mathrm{d} x \mathrm{~d} y
$$

(since geodesics on the periodic cube $D$ satisfy $\Gamma(t, x, y)-x=\Gamma(t, 0, y-x)$ )

$$
=\int_{D^{2}} \partial_{t} \Gamma(t, 0, y) H(x+\Gamma(t, 0, y)) \mathrm{d} x \mathrm{~d} y
$$

(using the change of variable $y \rightarrow y-x$ for each fixed $x$ and the translation invariance of the Lebesgue measure on $D)$

$$
=\int_{D^{2}} \partial_{t} \Gamma(t, 0, y) H(x) \mathrm{d} x \mathrm{~d} y
$$

(using the change of variable $x+\Gamma(t, 0, y) \rightarrow x$ for each fixed $y$ and again the translation invariance of the Lebesgue measure on $D$ )

$$
=\int_{D} \partial_{t} \Gamma(t, 0, y) \mathrm{d} y \int_{D} H(x) \mathrm{d} x=0
$$

(by symmetry of geodesic curves on $D$ with respect to reflections). The case $T / 2 \leq t \leq T$ can be treated similarly, as well as the general case when $u$ is not null. Finally, the Action can be immediately bounded by

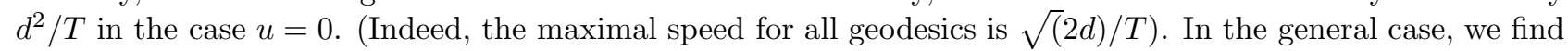

$$
K(c, m) \leq C\left(\|u\|_{L^{2}}^{2}+\frac{d^{2}}{T}+\left\|\nabla_{x} X\right\|_{L^{\infty}}^{2}\right) .
$$

\section{Duality, approximate Lagrange multipliers}

In this section, we prove Proposition 2.1. We use a duality argument which implies that the infimum is reached and that there exists a sequence $\left(\phi_{\varepsilon}, H_{\varepsilon}\right)$ satisfying $(11)$ and (12). We consider those inequalities as an approximation of

$$
\partial_{t} \phi+\frac{1}{2}\left|\nabla_{x} \phi+H\right|^{2}=0
$$

with

$$
v=\nabla_{x} \phi+H
$$

which can be seen as the integral (in $x$ ) version of

$$
\partial_{t} v+v \cdot \nabla v-\partial_{t} H+v \wedge \operatorname{curl} H=0 .
$$

Proof. Let us introduce two convex functions $\alpha$ and $\beta$ defined on $\mathcal{E}=C\left(Q^{\prime}\right) \times C\left(Q^{\prime}\right)^{d}$ with values in ] $\left.-\infty, \infty\right]$ given by

$$
\alpha(F ; \Phi)=\left\{\begin{array}{l}
0 \text { if } F+\frac{1}{2}|\Phi|^{2} \leq 0 \\
\infty \text { elsewhere }
\end{array}\right.
$$




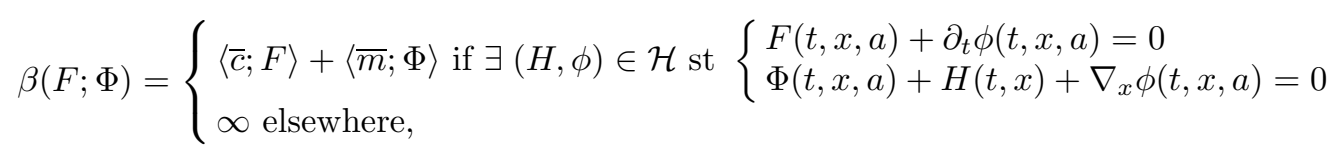

where $(\bar{c} ; \bar{m})$ is an arbitrary admissible pair and $\mathcal{H}=C\left(Q ; \mathbb{R}^{d}\right) \times C^{1,1,0}\left(Q^{\prime}\right)$.

Notice that the definition of $\beta$ does not depend on the admissible pair $(\bar{c} ; \bar{m})$.

Their dual functions [11] are defined by

$$
\begin{aligned}
& \alpha^{*}(c, m)=\sup _{F, \Phi}\langle c ; F\rangle+\langle m ; \Phi\rangle \text { with } F+\frac{1}{2}|\Phi|^{2} \leq 0, \\
& \beta^{*}(c, m)=\sup _{F, \Phi}\langle c-\bar{c} ; F\rangle+\langle m-\bar{m} ; \Phi\rangle \text { with }(F, \Phi) \in A_{d},
\end{aligned}
$$

where $A_{d}=\left\{(F, \Phi): Q \rightarrow \mathbb{R} \times \mathbb{R}^{d}\right.$ such that $\exists(H, \phi) \in \mathcal{H}$ st $\left\{\begin{array}{l}F(t, x, a)+\partial_{t} \phi(t, x, a)=0 \\ \Phi(t, x, a)+H(t, x)+\nabla_{x} \phi(t, x, a)=0\end{array}\right\}$.

Remark 4.1. Function $\beta^{*}$ only takes values $+\infty$ and 0 . In the later case, we have for every $F$ and $\Phi \in A_{d}$

$$
\langle c ; F\rangle=\langle\bar{c} ; F\rangle \text { and }\langle m ; \Phi\rangle=\langle\bar{m} ; \Phi\rangle
$$

and then, for every $(H, \phi) \in \mathcal{H}$ :

$$
\langle m-\bar{m} ; H\rangle=0 \text { and }\left\langle c-\bar{c} ; \partial_{t} \phi\right\rangle+\left\langle m-\bar{m} ; \nabla_{x} \phi\right\rangle=0,
$$

which exactly means that $(c, m)$ is admissible (i.e. satisfies (7) and (8)).

Thanks to the Fenchel-Rockafellar theorem [11], we have

$$
\begin{aligned}
& \min \left\{\alpha^{*}(c, m)+\beta^{*}(c, m) ;(c, m) \in \mathcal{E}^{\prime}, \text { the dual space of } \mathcal{E}\right\} \\
& =\sup \{-\alpha(-F ;-\Phi)-\beta(F ; \Phi) ;(F ; \Phi) \in \mathcal{E}\} .
\end{aligned}
$$

This ensures that the infimum (that we already know to be finite) is achieved. Indeed, we have an admissible pair with finite Action, which means

$$
\alpha^{*}(c, m)+\beta^{*}(c, m)=K(c, m)<\infty .
$$

Let us now reconstruct $\alpha$ and $\beta$ starting from $(\bar{c}, \bar{m})$, an optimal solution. We obtain

$$
I\left(c_{0}, c_{T}\right)=K(\bar{c}, \bar{m})=\sup \{-\alpha(-F ;-\Phi)-\beta(F ; \Phi) ;(F ; \Phi) \in \mathcal{E}\} .
$$

This equality implies that for every $\varepsilon>0$, there exist $\left(H_{\varepsilon}, \phi_{\varepsilon}\right) \in \mathcal{H}$ so that

$$
\partial_{t} \phi_{\varepsilon}+\frac{1}{2}\left|\nabla_{x} \phi_{\varepsilon}+H_{\varepsilon}\right|^{2} \leq 0
$$

and

$$
\frac{1}{2}\left\langle\bar{c} ;|v|^{2}\right\rangle \leq\left\langle\bar{c} ; \partial_{t} \phi_{\varepsilon}\right\rangle+\left\langle\bar{m} ; \nabla_{x} \phi_{\varepsilon}+H_{\varepsilon}\right\rangle+\varepsilon^{2} .
$$

Moreover, thanks to (17), those inequalities are true for every optimal solution. Finally, there exist $\phi_{\varepsilon}$ and $H_{\varepsilon}$ satisfying

$$
\partial_{t} \phi_{\varepsilon}+\frac{1}{2}\left|\nabla_{x} \phi_{\varepsilon}+H_{\varepsilon}\right|^{2} \leq 0
$$


such that for every optimal solution, we have

$$
\frac{1}{2}\left\langle\bar{c} ;\left|v-\left(\nabla_{x} \phi_{\varepsilon}+H_{\varepsilon}\right)\right|^{2}\right\rangle+\left.\left\langle\bar{c} ;\left|\partial_{t} \phi_{\varepsilon}+\frac{1}{2}\right| \nabla_{x} \phi_{\varepsilon}+H_{\varepsilon}\right)\right|^{2}|\rangle \leq \varepsilon^{2}
$$

\section{Preliminary estimates}

Let $\delta$ and $\eta$ be two small parameters and let us assume that $0<\tau<\frac{T}{2}$. Let $\zeta(t)$ be a smooth function compactly supported for $0<t<T$. We choose $\eta$ small enough so that $t \rightarrow t+\eta \zeta(t)$ is a diffeomorphism from $[0, T]$ to $[0, T]$. We shall denote $\tau_{\eta}(t)$ its inverse. Let $x \rightarrow w(x)$, be a smooth vector field and $e^{s w}(x)$ the flow associated to $w(x)$ defined by

$$
\partial_{s} \mathrm{e}^{s w}(x)=w\left(\mathrm{e}^{s w}(x)\right) \text { and } \mathrm{e}^{0 w}(x)=x,
$$

we introduce, as in [9], the following measures

$$
c^{\eta}(t, x, a)=c(t+\eta \zeta(t), x, a), v^{\eta}(t, x, a)=v(t+\eta \zeta(t), x, a)\left(1+\eta \zeta^{\prime}(t)\right)
$$

that we define precisely by

$$
\int_{Q^{\prime}} f(t, x, a) \mathrm{d} c^{\eta}=\int_{Q^{\prime}} f\left(\tau_{\eta}(t), x, a\right) \tau_{\eta}^{\prime}(t) \mathrm{d} c(t, x, a) \text { and } \int_{Q^{\prime}} f(t, x, a) \mathrm{d} m^{\eta}=\int_{Q^{\prime}} f\left(\tau_{\eta}(t), x, a\right) \mathrm{d} m(t, x, a) .
$$

for every function $f \in C\left(Q^{\prime}\right)$. Then we define the measures $\left(c^{\eta, \delta}, m^{\eta, \delta}\right)$ so that for every $f \in C\left(Q^{\prime}\right)$

$$
\int_{Q^{\prime}} f(t, x, a) \mathrm{d} c^{\eta, \delta}(t, x, a)=\int_{Q^{\prime}} f\left(t, \mathrm{e}^{\delta \zeta(t) w}(x), a\right) \mathrm{d} c^{\eta}
$$

and

$$
\int_{Q^{\prime}} f(t, x, a) \mathrm{d} m^{\eta, \delta}(t, x, a)=\int_{Q^{\prime}} f\left(t, \mathrm{e}^{\delta \zeta(t) w}(x), a\right)\left(\partial_{t}+v^{\eta}(t, x, a) \cdot \nabla_{x}\right) \cdot \mathrm{e}^{\delta \zeta(t) w}(x) \mathrm{d} c^{\eta}
$$

Remark 5.1. If the pair $\left(c^{\eta \delta}, m^{\eta \delta}\right)$ is constructed from an admissible pair, we have $\int c^{\eta, \delta}(t, x, \mathrm{~d} a)=1$ as soon as $w(x)$ is divergence free and the pair $\left(c^{\eta \delta}, m^{\eta \delta}\right)$ satisfies $(6)$.

Proposition 5.1. Let $(c, m)$ be an optimal solution, for any $\eta$ and any $\delta$, we have the following inequality:

$$
\begin{aligned}
& \int_{Q^{\prime}} H_{\varepsilon} \cdot\left(\mathrm{d} m^{\eta, \delta}-\mathrm{d} m\right)+\frac{1}{2} \int_{Q^{\prime}}\left|\left(\partial_{t}+v^{\eta} \cdot \nabla_{x}\right) \mathrm{e}^{\delta \zeta(t) w}-\left(\nabla_{x} \phi_{\varepsilon}+H_{\varepsilon}\right) \circ \mathrm{e}^{\delta \zeta(t) w}\right|^{2} \mathrm{~d} c^{\eta} \\
& \leq \varepsilon^{2}+\frac{1}{2} \int_{Q^{\prime}}\left|\left(\partial_{t}+v^{\eta} \cdot \nabla_{x}\right) \mathrm{e}^{\delta \zeta(t) w}\right|^{2} \mathrm{~d} c^{\eta}-\frac{1}{2} \int_{Q^{\prime}}|v|^{2} \mathrm{~d} c .
\end{aligned}
$$

Proof. From the relation (7) between the measures $c$ and $m$, we have

$$
\int_{Q^{\prime}} \partial_{t} \phi_{\varepsilon}\left(\mathrm{d} c^{\eta, \delta}-\mathrm{d} c\right)=-\int_{Q^{\prime}} \nabla_{x} \phi_{\varepsilon} \cdot\left(\mathrm{d} m^{\eta, \delta}-\mathrm{d} m\right)
$$


because since $\zeta$ is compactly supported in time, the boundary terms in time of (7) vanish. Then, we obtain

$$
\begin{aligned}
\int_{Q^{\prime}} \partial_{t} \phi_{\varepsilon}\left(\mathrm{d} c^{\eta, \delta}-\mathrm{d} c\right)= & -\int_{Q^{\prime}}\left(\partial_{t}+v^{\eta} \cdot \nabla_{x}\right) \mathrm{e}^{\delta \zeta(t) w} \cdot \nabla_{x} \phi_{\varepsilon} \circ \mathrm{e}^{\delta \zeta(t) w} \mathrm{~d} c^{\eta}+\int_{Q^{\prime}} v \cdot \nabla_{x} \phi_{\varepsilon} \mathrm{d} c \\
= & -\int_{Q^{\prime}}\left(\partial_{t}+v^{\eta} \cdot \nabla_{x}\right) \mathrm{e}^{\delta \zeta(t) w} \cdot\left(\nabla_{x} \phi_{\varepsilon}+H_{\varepsilon}\right) \circ \mathrm{e}^{\delta \zeta(t) w} \mathrm{~d} c^{\eta}+\int_{Q^{\prime}} v \cdot\left(\nabla_{x} \phi_{\varepsilon}+H_{\varepsilon}\right) \mathrm{d} c \\
& +\int_{Q^{\prime}} H_{\varepsilon} \cdot\left(\mathrm{d} m^{\eta, \delta}-\mathrm{d} m\right) .
\end{aligned}
$$

After a systematic transformation of the products, we obtain

$$
\begin{aligned}
\int_{Q^{\prime}} \partial_{t} \phi_{\varepsilon}\left(\mathrm{d} c^{\eta, \delta}-\mathrm{d} c\right)= & -\frac{1}{2} \int_{Q^{\prime}}\left|\left(\partial_{t}+v^{\eta} \cdot \nabla_{x}\right) \mathrm{e}^{\delta \zeta(t) w}\right|^{2} \mathrm{~d} c^{\eta} \\
& +\frac{1}{2} \int_{Q^{\prime}}\left|\left(\partial_{t}+v^{\eta} \cdot \nabla_{x}\right) \mathrm{e}^{\delta \zeta(t) w}-\left(\nabla_{x} \phi_{\varepsilon}+H_{\varepsilon}\right) \circ \mathrm{e}^{\delta \zeta(t) w}\right|^{2} \mathrm{~d} c^{\eta} \\
& -\frac{1}{2} \int_{Q^{\prime}}\left|\nabla_{x} \phi_{\varepsilon}+H_{\varepsilon}\right|^{2} \mathrm{~d} c^{\eta, \delta}-\frac{1}{2} \int_{Q^{\prime}}\left|\nabla_{x} \phi_{\varepsilon}+H_{\varepsilon}-v\right|^{2} \mathrm{~d} c \\
& +\frac{1}{2} \int_{Q^{\prime}}\left|\nabla_{x} \phi_{\varepsilon}+H_{\varepsilon}\right|^{2} \mathrm{~d} c+\frac{1}{2} \int_{Q^{\prime}} v^{2} \mathrm{~d} c+\int_{Q^{\prime}} H_{\varepsilon} \cdot\left(\mathrm{d} m^{\eta, \delta}-\mathrm{d} m\right) .
\end{aligned}
$$

Hence, we have the following equality

$$
\begin{aligned}
& \int_{Q^{\prime}} H_{\varepsilon} \cdot\left(\mathrm{d} m^{\eta, \delta}-\mathrm{d} m\right)+\frac{1}{2} \int_{Q^{\prime}}\left|\left(\partial_{t}+v^{\eta} \cdot \nabla_{x}\right) \mathrm{e}^{\delta \zeta(t) w}-\left(\nabla_{x} \phi_{\varepsilon}+H_{\varepsilon}\right) \circ \mathrm{e}^{\delta \zeta(t) w}\right|^{2} \mathrm{~d} c^{\eta} \\
& -\frac{1}{2} \int_{Q^{\prime}}\left|\left(\partial_{t}+v^{\eta} \cdot \nabla_{x}\right) \mathrm{e}^{\delta \zeta(t) w}\right|^{2} \mathrm{~d} c^{\eta}+\frac{1}{2} \int_{Q^{\prime}}|v|^{2} \mathrm{~d} c \\
& =\int_{Q^{\prime}}\left(\partial_{t} \phi_{\varepsilon}+\frac{1}{2}\left|\nabla_{x} \phi_{\varepsilon}+H_{\varepsilon}\right|^{2}\right)\left(\mathrm{d} c^{\eta, \delta}-\mathrm{d} c\right)+\frac{1}{2} \int_{Q^{\prime}}\left|\nabla_{x} \phi_{\varepsilon}+H_{\varepsilon}-v\right|^{2} \mathrm{~d} c .
\end{aligned}
$$

Here, we use the properties (2.1) of $H_{\varepsilon}$ and $\phi_{\varepsilon}$ to estimate the right-hand side term by $\varepsilon^{2}$ noticing that

$$
\int_{Q^{\prime}}\left(\partial_{t} \phi_{\varepsilon}+\frac{1}{2}\left|\nabla_{x} \phi_{\varepsilon}+H_{\varepsilon}\right|^{2}\right) \mathrm{d} c^{\eta, \delta} \leq 0
$$

and

$$
-\int_{Q^{\prime}}\left(\partial_{t} \phi_{\varepsilon}+\frac{1}{2}\left|\nabla_{x} \phi_{\varepsilon}+H_{\varepsilon}\right|^{2}\right) \mathrm{d} c+\frac{1}{2} \int_{Q^{\prime}}\left|\nabla_{x} \phi_{\varepsilon}+H_{\varepsilon}-v\right|^{2} \mathrm{~d} c \leq \varepsilon^{2} .
$$

So we obtain inequality (5.1).

In the following, we first make a restrictive assumption on the total momentum $u$. We assume that

$$
u(t, x)=\frac{1}{2} \zeta_{u}^{\prime}(t) U\left(\zeta_{u}(t), x\right) \text { with } U(t, x) \in C^{\infty}([0, T] \times D) \text { and } \zeta_{u}(t) \in C_{c}^{\infty}(] 0, T[) .
$$


Remark 5.2. To obtain the complete result, we have to take $u=0$, so the reader principally interested in the final result can consider only this case.

Assumption (20) is in fact a sufficient assumption so that the flow associated to $u, X$, satisfies $X(T, x)=$ $X(0, x)=x$. Moreover, the Cauchy-Lipschitz theorem ensures that this flow can be reversed.

In this framework, we use a more general formulation of (5.1). Indeed, the same proof as the one presented above leads to the following proposition:

Proposition 5.2. For any transformation $\bar{M}(t, x)$ that satisfies

$$
\bar{M}(0, x)=x \text { and } \bar{M}(T, x)=x
$$

we have

$$
\begin{aligned}
& \int_{Q} H_{\varepsilon}(t, \bar{M}(t, x))\left(\partial_{t} \bar{M}(t, x)+u(t, x) \cdot \nabla \bar{M}(t, x)\right) \mathrm{d} t \mathrm{~d} x-\int H_{\varepsilon}(t, x) u(t, x) \mathrm{d} t \mathrm{~d} x \\
& +\frac{1}{2} \int_{Q^{\prime}}\left|\left(\partial_{t}+v \cdot \nabla\right) \bar{M}(t, x)-\left(\nabla \phi_{\varepsilon}+H_{\varepsilon}\right)(t, \bar{M}(t, x), a)\right|^{2} \mathrm{~d} c \\
& \leq \varepsilon^{2}+\frac{1}{2} \int_{Q^{\prime}}\left|\left(\partial_{t}+v \cdot \nabla_{x}\right) \bar{M}(t, x)\right|^{2} \mathrm{~d} c-\frac{1}{2} \int_{Q^{\prime}}|v|^{2} \mathrm{~d} c .
\end{aligned}
$$

Proof. To obtain (21), it is enough to see that

$$
\begin{aligned}
\int_{Q^{\prime}} \partial_{t} \phi_{\varepsilon}(t, \bar{M}(t, x), a) \mathrm{d} c-\int_{Q^{\prime}} \partial_{t} \phi_{\varepsilon}(t, x, a) \mathrm{d} c= & -\int_{Q^{\prime}}\left(\partial_{t}+v \cdot \nabla_{x}\right) \bar{M}(t, x) \cdot \nabla_{x} \phi_{\varepsilon}(\bar{M}(t, x)) \mathrm{d} c \\
& +\int_{Q^{\prime}} v \cdot \nabla_{x} \phi_{\varepsilon} \mathrm{d} c
\end{aligned}
$$

which comes from the integrated continuity equation (7). Indeed, we have

$$
\begin{aligned}
& \int_{Q^{\prime}} \partial_{t}\left(\phi_{\varepsilon}(t, \bar{M}(t, x), a)\right) \mathrm{d} c+\int_{Q^{\prime}} v \cdot \nabla\left(\phi_{\varepsilon}(t, \bar{M}(t, x), a)\right) \mathrm{d} c \\
& =\int_{D}\left(\phi_{\varepsilon}(T, \bar{M}(T, x), a) \mathrm{d} c_{T}(x, a)-\phi_{\varepsilon}(0, \bar{M}(0, x), a)\right) \mathrm{d} c_{0}(x, a) \\
& =\int_{Q^{\prime}} \partial_{t}\left(\phi_{\varepsilon}(t, x, a)\right) \mathrm{d} c+\int_{Q^{\prime}} v \cdot \nabla\left(\phi_{\varepsilon}(t, x, a)\right) \mathrm{d} c
\end{aligned}
$$

since $\bar{M}(T, x)=x$. The continuation of the proof is similar to the previous case.

We will also use the following proposition

Proposition 5.3. For every transformation $M(t, x)$ that satisfy

$$
M(0, x)=x \text { and } M(T, x)=x,
$$

we have

$$
\int H_{\varepsilon}(t, M(t, x)) \partial_{t} M(t, x) \mathrm{d} t \mathrm{~d} x-\int H_{\varepsilon}(t, x) u(t, x) \mathrm{d} t \mathrm{~d} x \leq 1+C\left(\left\|\partial_{t} M\right\|_{L^{2}}^{2}+\|\nabla M\|_{L^{\infty}}^{2}\right) .
$$


Proof. Let $\bar{M}$ be the function satisfying $M(t, x)=\bar{M}(t, X(t, x))$, we notice that

$$
\int H_{\varepsilon}(t, M(t, x)) \partial_{t} M(t, x) \mathrm{d} t \mathrm{~d} x=\int_{Q} H_{\varepsilon}(t, \bar{M}(t, x))\left(\partial_{t} \bar{M}(t, x)+u \cdot \nabla \bar{M}(t, x)\right) \mathrm{d} t \mathrm{~d} x
$$

since $X$ is Lebesgue measure preserving. Since $X(0, x)=X(T, x)=x$, we have

$$
\bar{M}(0, x)=x \text { and } \bar{M}(T, x)=x
$$

and then, thanks to (21), we obtain

$$
\begin{aligned}
\int H_{\varepsilon}(t, M(t, x)) \partial_{t} M(t, x) \mathrm{d} t \mathrm{~d} x-\int H_{\varepsilon}(t, x) u(t, x) \mathrm{d} t \mathrm{~d} x \leq & \varepsilon^{2}+\frac{1}{2} \int_{Q^{\prime}}\left|\left(\partial_{t}+v \cdot \nabla_{x}\right) \bar{M}(t, x)\right|^{2} \mathrm{~d} c \\
& -\frac{1}{2} \int_{Q^{\prime}}|v|^{2} \mathrm{~d} c \\
\leq & 1+C\left(\left\|\partial_{t} M\right\|_{L^{2}}^{2}+\|\nabla M\|_{L^{\infty}}^{2}\right) .
\end{aligned}
$$

Indeed, since the Jacobian of $X$ is equal to 1 , we have

$$
\int\left|\partial_{t} M\left(t, X^{-1}(t, x)\right)\right|^{2} \mathrm{~d} t \mathrm{~d} x=\int\left|\partial_{t} M(t, x)\right|^{2} \mathrm{~d} t \mathrm{~d} x .
$$

Remark 5.3. Let $w$ be the vector field satisfying

$$
\begin{aligned}
& \partial_{t} M(t, x)=w(t, M(t, x)) \\
& M(0, x)=x,
\end{aligned}
$$

inequality (23) becomes

$$
\int H_{\varepsilon}(t, M(t, x)) w(t, M(t, x)) \mathrm{d} t \mathrm{~d} x-\int H_{\varepsilon}(t, x) u(t, x) \mathrm{d} t \mathrm{~d} x \leq 1+C\left(\left\|\partial_{t} M\right\|_{L^{2}}^{2}+\|\nabla M\|_{L^{\infty}}^{2}\right)
$$

or

$$
\int H_{\varepsilon}(t, x)(w(t, x) \sigma(t, x)-u(t, x)) \mathrm{d} t \mathrm{~d} x \leq C\left(1+\left\|\partial_{t} M\right\|_{L^{2}}^{2}+\|\nabla M\|_{L^{\infty}}^{2}\right)
$$

where $\sigma$ is the Jacobian associated to $M$ which is linked to $w$ through

$$
\partial_{t} \sigma+\operatorname{div}(\sigma w)=0 \text { and } \sigma(0, x)=1 .
$$

\section{First BOUnds FOR $\mathrm{H}_{\varepsilon}$}

In this section, we show that $H_{\varepsilon}$ is bounded in a suitable distribution space, up to a time independent gradient, i.e. that the time derivative and the curl of $H_{\varepsilon}$ are bounded. The main difficulty of our analysis is that in order to derive the optimality equations we must be able to pass to the limit in expressions such as $\left\langle\partial_{t} H_{\varepsilon} ; g\right\rangle$ and $\left\langle\operatorname{curl} H_{\varepsilon} ; g\right\rangle$ not only for compactly supported functions $g(t, x)$ of $0<t<T, x \in D$, with unlimited regularity, but also for those with limited regularity, namely with a finite norm in $G_{m_{0}}=H^{1, m_{0}}([0, T] \times D)$ for some integer $m_{0}$ large enough, which means bounded derivatives in $L^{2}$ for high order derivatives in $x$ but only first order derivatives in $t$. 


\subsection{An estimate for $\partial_{t} H_{\varepsilon}$}

In this part, we use assumption (20) on the total momentum $u$.

First case: Test functions that are tensor products

We show the following proposition:

Proposition 6.1. There exist $C, r_{0}>0$ and an integer $m_{0}$ so that for every smooth vector field a $(t, x)=$ $\zeta(t) A(x)$ with $A \in C^{\infty}(D)$ and $\zeta \in C_{c}^{\infty}(] 0, T[),\|a\|_{G_{m_{0}}} \leq r_{0}$ implies

$$
\int H_{\varepsilon}(t, x) \partial_{t} a(t, x) \mathrm{d} t \mathrm{~d} x \leq C
$$

Proof of Proposition 6.1. The main idea of the proof consists in finding $M$ so that

$$
\int H_{\varepsilon}(t, x) \partial_{t} a(t, x)=\int H_{\varepsilon}(t, M(t, x)) \partial_{t} M(t, x) \mathrm{d} t \mathrm{~d} x
$$

and then using (23). In order to use (23), in the case when $u$ is not null, we first need a bound for

$$
\left|\int H_{\varepsilon}(t, x) u(t, x) \mathrm{d} t \mathrm{~d} x\right|
$$

and use (24).

We note that assumption (20) ensures the existence of $M_{u}$ satisfying (22) so that

$$
\int H_{\varepsilon}\left(t, M_{u}(t, x)\right) \partial_{t} M_{u}(t, x) \mathrm{d} t \mathrm{~d} x=2 \int H_{\varepsilon}(t, x) u(t, x) \mathrm{d} t \mathrm{~d} x .
$$

Indeed, let us define $\tilde{M}_{u}$ by

$$
\partial_{t} \tilde{M}_{u}(t, x)=U\left(t, \tilde{M}_{u}(t, x)\right) \text { and } \tilde{M}_{u}(0, x)=x,
$$

we have (27) with $M_{u}(t, x)=\tilde{M}_{u}\left(\zeta_{u}(t), x\right)$ which satisfies $M_{u}(T, x)=M_{u}(0, x)=x$. Indeed, we have

$$
\begin{aligned}
\int H_{\varepsilon}\left(t, M_{u}(t, x)\right) \partial_{t} M_{u}(t, x) \mathrm{d} t \mathrm{~d} x & =\int H_{\varepsilon}\left(t, \tilde{M}_{u}\left(\zeta_{u}(t), x\right)\right) \zeta_{u}^{\prime}(t) U\left(\zeta_{u}(t), \tilde{M}_{u}\left(\zeta_{u}(t), x\right)\right) \mathrm{d} t \mathrm{~d} x \\
& =\int H_{\varepsilon}(t, x) \zeta_{u}^{\prime}(t) U\left(\zeta_{u}(t), x\right) \mathrm{d} t \mathrm{~d} x \\
& =2 \int H_{\varepsilon}(t, x) u(t, x) \mathrm{d} t \mathrm{~d} x
\end{aligned}
$$

Remark 6.1. Let $\tilde{\sigma}$ be the Jacobian of $\tilde{M}_{u}$, we know that $\partial_{t} \tilde{\sigma}+\operatorname{div}(\tilde{\sigma} U)=0$. Because $u$ is divergence free, $U$ is also divergence free and therefore $\tilde{\sigma}=1$, which means that $\tilde{M}_{u}$ is Lebesgue measure-preserving.

Using (23), we obtain

$$
\int H_{\varepsilon}(t, x) u(t, x) \mathrm{d} t \mathrm{~d} x \leq C
$$

and the estimate

$$
-\int H_{\varepsilon}(t, x) u(t, x) \mathrm{d} t \mathrm{~d} x \leq C
$$

comes directly from (23) in the special case $M(t, x)=x$. 
Now, if there is $M$ satisfying (26), by using (23) we deduce

$$
\int H_{\varepsilon}(t, x) \partial_{t} a(t, x) \mathrm{d} t \mathrm{~d} x=\int H_{\varepsilon}(t, M(t, x)) \partial_{t} M(t, x) \mathrm{d} t \mathrm{~d} x \leq C\left(1+\left\|\partial_{t} M\right\|_{L^{2}}^{2}+\|\nabla M\|_{L^{\infty}}^{2}\right) .
$$

Let us now find $M$ so that

$$
\int H_{\varepsilon}(t, x) \partial_{t} a(t, x)=\int H_{\varepsilon}(t, M(t, x)) \partial_{t} M(t, x) \mathrm{d} t \mathrm{~d} x
$$

To be sure that $M$ satisfies (22), we look for it in the following way

$$
M(t, x)=\tilde{M}(\zeta(t), x) \text { where } \zeta \in C_{c}^{\infty}(] 0, T[) .
$$

Let $S=\left[s_{0}, s_{1}\right]$ the range of $\zeta(t)$ for $0 \leq t \leq T$. We set for $s \in S$

$$
\begin{aligned}
& \tilde{\sigma}(s, x)=1-s \nabla \cdot A(x) \\
& \tilde{w}(s, x)=\frac{A(x)}{1-s \nabla \cdot A(x)}, \\
& \partial_{s} \tilde{M}(s, x)=\tilde{w}(s, \tilde{M}(s, x)), \quad \tilde{M}(0, x)=x .
\end{aligned}
$$

By choosing $r_{0}>0$ small enough, we get

$$
\sup _{s \in S, x \in D}|s \nabla \cdot A(x)|=\sup _{t \in[0, T], x \in D}|\zeta(t) \nabla \cdot A(x)|=\|\nabla \cdot a\|_{L^{\infty}} \leq \frac{1}{2}
$$

and, then, $\tilde{w}$ is well defined and smooth. To guarantee that $M$ satisfies (22), we set

$$
M(t, x)=\tilde{M}(\zeta(t), x) .
$$

From these definitions, we get

$$
\partial_{t} \tilde{\sigma}+\nabla \cdot(\tilde{\sigma} \tilde{w})=0
$$

and

$$
\int H_{\varepsilon}(t, M(t, x)) \partial_{t} M(t, x) \mathrm{d} t \mathrm{~d} x=\int H_{\varepsilon}(t, x) \zeta^{\prime}(t) \tilde{w}(\zeta(t), x) \tilde{\sigma}(\zeta(t), x) \mathrm{d} t \mathrm{~d} x=\int H_{\varepsilon}(t, x) \partial_{t} a(t, x) \mathrm{d} t \mathrm{~d} x .
$$

To conclude the proof, it remains to estimate $\left(\left\|\partial_{t} M\right\|_{L^{2}}^{2}+\|\nabla M\|_{L^{\infty}}^{2}\right)$ in terms of the norm of $a$ in $G=$ $H^{1, m}([0, T] \times D)$.

Let us compute

$$
\begin{aligned}
\int\left|\partial_{t} M(t, x)\right|^{2} \mathrm{~d} t \mathrm{~d} x & =\int \zeta^{\prime}(t)^{2}|\tilde{w}(\zeta(t), M(t, x))|^{2} \mathrm{~d} t \mathrm{~d} x \\
& =\int \zeta^{\prime}(t)^{2}|\tilde{w}(\zeta(t), x)|^{2} \tilde{\sigma}(\zeta(t), x) \mathrm{d} t \mathrm{~d} x
\end{aligned}
$$

which becomes, after replacing $\tilde{w}$ and $\tilde{\sigma}$ by their explicit values,

$$
\int\left|\partial_{t} M(t, x)\right|^{2} \mathrm{~d} t \mathrm{~d} x=\int \zeta^{\prime}(t)^{2} \frac{|A(x)|^{2}}{1-\nabla \cdot(\zeta(t) A(x))} \mathrm{d} t \mathrm{~d} x .
$$


Thus,

Similarly, we deduce from

$$
\int\left|\partial_{t} M(t, x)\right|^{2} \mathrm{~d} t \mathrm{~d} x \leq \int 2 \zeta^{\prime}(t)^{2}|A(x)|^{2} \mathrm{~d} t \mathrm{~d} x=2\left\|\partial_{t} a\right\|_{L^{2}}^{2} .
$$

that

$$
\partial_{t} M(t, x)=\zeta^{\prime}(t) \tilde{w}(\zeta(t), M(t, x))
$$

and we know that $M(0, x)=x$. Thus

$$
\partial_{t} \partial_{j} M_{i}(t, x)=\zeta^{\prime}(t) \partial_{j} M_{k}(t, x) \partial_{k} \tilde{w}_{i}(\zeta(t), M(t, x)),
$$

$$
|\nabla M(t, x)| \leq C \exp \left(\left.\int_{0}^{T}\left|\zeta^{\prime}(s)\right||| \nabla \tilde{w}(\zeta(s), .)\right|_{L^{\infty}(D)} \mathrm{d} s\right) .
$$

Let us estimate the right-hand side of this inequality. We have

$$
\partial_{j} \tilde{w}_{i}(\zeta(t), x)=\frac{\partial_{j} A_{i}(x)}{1-\zeta(t) \nabla \cdot A(x)}+\zeta(t) \frac{A_{i}(x) \partial_{j}(\nabla \cdot A(x))}{(1-\zeta(t) \nabla \cdot A(x))^{2}} .
$$

If $r_{0}>0$ is chosen small enough, by assumption, we get

$$
|\zeta(t) \nabla \cdot A(x)| \leq\|\nabla \cdot a\|_{L^{\infty}} \leq \frac{1}{2}
$$

and

Thus

$$
|\zeta(t) \nabla(\nabla \cdot A(x))| \leq\|\zeta\|_{L^{\infty}}\|\nabla(\nabla \cdot A)\|=\|\nabla(\nabla \cdot a)\|_{L^{\infty}} \leq 1 .
$$

It follows that

$$
|\nabla \tilde{w}(\zeta(t), x)| \leq C(|A(x)|+|\nabla A(x)|)
$$

$$
\begin{aligned}
|\nabla M(t, x)| & \leq C \exp \left(C \int_{0}^{T}\left|\zeta^{\prime}(s)\right| \mathrm{d} s\left(\|A\|_{L^{\infty}}+\|\nabla A\|_{L^{\infty}}\right)\right) . \\
& \leq C \exp \left(C\|||\|_{G_{m_{0}}}\right)
\end{aligned}
$$

for $m_{0}$ large enough (more precisely for $m_{0}-d / 2>1$ ), which completes the proof.

Second case: General test functions

Proposition 6.2. There exist a constant $C$ and an integer $m_{0}$ (that do not depend on $\varepsilon$ ) so that for every smooth vector field $a(t, x) \in C_{c}^{\infty}(] 0, T[\times D)$,

$$
\int H_{\varepsilon}(t, x) \partial_{t} a(t, x) \mathrm{d} t \mathrm{~d} x \leq C\|a\|_{G_{m_{0}}}
$$

This proposition immediately follows from:

Lemma 6.3. Let $T_{\varepsilon}(t, x)$ be a family of distributions for $x \in D$ and $\left.t \in\right] 0, T[$, satisfying, for some constant $C$ and some integer $m_{0}$,

$$
\left|\left\langle T_{\varepsilon}, \phi\right\rangle\right| \leq C \sum_{l \leq 1,|\alpha| \leq m_{0}}\left\|\partial_{t}^{l} \partial^{\alpha} \phi\right\|_{L^{2}}
$$

for all tensor products $\phi(t, x)=\phi_{0}(t) \phi_{1}(x)$. Then (28) remains true for all test functions provided $m_{0}$ is taken larger. 
Proof. The proof of this lemma can be made using the Fourier transform. Let us denote $\langle k\rangle=\sqrt{1+|k|^{2}}$. Let $\phi$ be a test function belonging to $C_{c}^{\infty}(] 0, T[\times D)$, we can write

$$
\phi(t, x)=\sum_{k \in \mathbb{Z}^{d}} \hat{\phi}(t, k) \mathrm{e}^{2 i \pi k \cdot x}
$$

Then

$$
\begin{aligned}
\left|\left\langle T_{\varepsilon}, \phi\right\rangle\right| & \leq \sum_{k \in \mathbb{Z}^{d}}\left|\left\langle T_{\varepsilon}, \hat{\phi}(t, k) \mathrm{e}^{2 i \pi k \cdot x}\right\rangle\right| \\
& \leq C \sum_{k \in \mathbb{Z}^{d}} \sum_{l \leq 1,|\alpha| \leq m_{0}}\left\|\partial_{t}^{l} \partial^{\alpha}\left(\hat{\phi}(t, k) \mathrm{e}^{2 i \pi k \cdot x}\right)\right\|_{L^{2}(Q)}
\end{aligned}
$$

and using (28), we have

$$
\begin{aligned}
\left|\left\langle T_{\varepsilon}, \phi\right\rangle\right| & \leq C \sum_{k \in \mathbb{Z}^{d}} \sum_{l \leq 1}\langle k\rangle^{m_{0}}\left\|\partial_{t}^{l} \hat{\phi}(t, k)\right\|_{L^{2}([0, T])} \\
& \leq C \sum_{l \leq 1}\left(\sqrt{\sum_{k \in \mathbb{Z}^{d}}\langle k\rangle^{2 m_{0}+r}\left\|\partial_{t}^{l} \hat{\phi}(t, k)\right\|_{L^{2}([0, T])}^{2}} \sqrt{\sum_{k \in \mathbb{Z}^{d}}\langle k\rangle^{-r}}\right) \text { where } r>d \\
& \leq C \sum_{l \leq 1,|\alpha| \leq m_{0}+\frac{r}{2}} \sqrt{\int\left|\partial_{t}^{l} \partial^{\alpha} \phi(t, x)\right|^{2} \mathrm{~d} t \mathrm{~d} t}
\end{aligned}
$$

thanks to the Parseval equality.

\subsection{An estimate for $\operatorname{curl} H_{\varepsilon}$}

In this section, we assume that the dimension $d$ is 3 and the velocity $u$ is equal to zero.

First case: Test functions that are tensor product

We show the following proposition:

Proposition 6.4. There exist a constant $C$ and an integer $m_{0}$ such that, for every test function $A(t, x)=$ $a_{1}\left(t, x_{1}\right) a_{2}\left(t, x_{2}\right) a_{3}\left(t, x_{3}\right)$ where $a_{i}\left(t, x_{i}\right)=\zeta(t) \psi_{i}\left(x_{i}\right), \psi_{i} \in C^{\infty}(\mathbb{T})$ and $\zeta \in C_{c}^{\infty}(] 0, T[)$ satisfying $\|\zeta\|_{L^{\infty}} \leq 1$ and $\left\|\partial_{i}^{l} \psi_{i}\right\|_{L^{\infty}} \leq \frac{1}{4}$ for $i=1,2,3, \quad l=0, \ldots, m_{0}+1$, so that for every $1 \leq k \leq 3$,

$$
\int \operatorname{curl} H_{\varepsilon k}(t, x) A(t, x) \mathrm{d} t \mathrm{~d} x \leq C\left(1+\left(\sum_{i} \sum_{l=1}^{m_{0}}\left\|\partial_{t} \partial_{i}^{l} a_{i}\right\|_{L^{2}}^{2}\right)^{\frac{1}{2}}\right) .
$$

Remark 6.2. The following analysis is again complicated because we need an estimate where $a$ is involved only through its $H^{1, m_{0}}([0, T] \times D)$ norm with only one time derivative. An estimate in $\mathcal{D}^{\prime}(] 0, T[\times D)$ would be much easier to obtain.

First, we prove the following lemma:

Lemma 6.5. For every tensor product

$$
A(t, x)=a_{1}\left(t, x_{1}\right) a_{2}\left(t, x_{2}\right) a_{3}\left(t, x_{3}\right)
$$


with $a_{i} \in C^{\infty}([0, T] \times[0,1])$ satisfying $\left\|\partial_{i} a_{i}\right\|_{L^{\infty}} \leq \frac{1}{4}$, there exists $\left(W_{1}\left(t, x_{1}\right), W_{2}\left(t, x_{2}\right), W_{3}\left(t, x_{3}\right)\right)$ belonging to $C^{\infty}([0, T] \times[0,1])$ so that

$$
a_{i}\left(t, x_{i}+W_{i}\left(t, x_{i}\right)\right)=W_{i}\left(t, x_{i}\right) \text { and }\left\|\partial_{i} W_{i}\right\|_{L^{\infty}} \leq \frac{1}{3}
$$

\section{Moreover}

1) if the functions $a_{i}$ are 1-periodic with respect to $x_{i}$, then the functions $W_{i}$ are also 1-periodic;

2) if the functions $a_{i}$ are of form $a_{i}(t, x)=\tilde{a}_{i}\left(\zeta(t), x_{i}\right)$ with $\zeta$ that does not depend on $i$ and $\tilde{a}_{i}\left(s, x_{i}\right)=s \psi_{i}\left(x_{i}\right)$, then each $W_{i}$ is equal to $W_{i}\left(t, x_{i}\right)=\tilde{W}_{i}\left(\zeta(t), x_{i}\right)$ where each $\tilde{W}$ is constructed from $\tilde{a}_{i}$ following (29);

3) for every $f(t, x)$, we have

$$
\int f(t, x) A(t, x) \mathrm{d} t \mathrm{~d} x=\int_{0}^{1} \mathrm{~d} \theta \int_{0}^{1} \mathrm{~d} \sigma \int_{0}^{1} \mathrm{~d} \tau \int f\left(t ; x_{1}+\theta W_{1} ; x_{2}+\sigma W_{2} ; x_{3}+\tau W_{3}\right) W_{1} W_{2} W_{3} \mathrm{~d} x \mathrm{~d} t
$$

Proof of Lemma 6.5. For every $f$, we want to write

$$
\int f(t, x) A(t, x) \mathrm{d} t \mathrm{~d} x
$$

in the following way

$$
I=\int f\left(t ; x_{1}+\theta W_{1} ; x_{2}+\sigma W_{2} ; x_{3}+\tau W_{3}\right) W_{1} W_{2} W_{3} \mathrm{~d} x \mathrm{~d} t \mathrm{~d} \theta \mathrm{d} \sigma \mathrm{d} \tau
$$

Let us perform the change of variable $\theta \rightarrow x_{i}+\theta W_{i}\left(t, x_{i}\right)=y_{i}$ for $x_{i}$ fixed. The Jacobian is then $\mathrm{d} y_{i}=$ $W_{i}\left(t, x_{i}\right) \mathrm{d} \theta$.

Then,

$$
\begin{aligned}
I & =\int\left(\prod_{i=1}^{3} \int_{y_{i}=x_{i}}^{x_{i}+W_{i}\left(t, x_{i}\right)} f(t ; y) \mathrm{d} y\right) \mathrm{d} x \mathrm{~d} t \\
& =\iint f(t ; y) \prod_{i=1}^{3}\left\{\int_{x_{i} \in \mathbb{R}}\left(Y\left(y_{i}-x_{i}\right)-Y\left(y_{i}-\left(x_{i}+W_{i}\left(t, x_{i}\right)\right)\right)\right) \mathrm{d} x_{i}\right\} \mathrm{d} y \mathrm{~d} t,
\end{aligned}
$$

where $Y$ denotes the Heaviside function. Thus, to obtain

$$
\iint f(t ; y) a_{1}\left(t, y_{1}\right) a_{2}\left(t, y_{2}\right) a_{3}\left(t, y_{3}\right) \mathrm{d} t \mathrm{~d} y
$$

we just have to invert, for every $i$, the relation

$$
a_{i}\left(t, y_{i}\right)=\int\left(Y\left(y_{i}-x_{i}\right)-Y\left(y_{i}-\left(x_{i}+W_{i}\left(t, x_{i}\right)\right)\right) \mathrm{d} x_{i}\right.
$$

For the sake of simplicity, we will omit the letter $i$ in the following argument. If we assume a priori $\left\|\partial_{z} W\right\|_{L^{\infty}}<1$, we just have to find $W$ so that

$$
a(t, z+W(t, z))=W(t, z)
$$


Indeed, if $W$ satisfies (30), we have

$$
a(t, z+W(t, z))=\int(Y(z+W(t, z)-x)-Y((z+W(t, z)-x-W(t, x)))) \mathrm{d} x
$$

Since $x+W(t, x)$ is strictly increasing in $x, Y(z+W(t, z)-x-W(t, x)))=Y(z-x)$ and we get

$$
a(t, z+W(t, z))=\int(Y(z+W(t, z)-x)-Y(z-x)) \mathrm{d} x=W(t, z) .
$$

Equation (31) is just a fixed point equation where $t$ is a parameter. We get a unique solution as soon as $\left|\partial_{x} a(t, x)\right|<1$. To get a more precise information, we differentiate (31) in $z$ and get

$$
\partial_{z} W(t, z)=\frac{\partial_{x} a(t, z+W(t, z))}{1-\partial_{x} a(t, z+W(t, z))}
$$

which shows that $\left\|\partial_{z} W\right\|_{L^{\infty}} \leq 1 / 3$ follows from $\left\|\partial_{x} a\right\|_{L^{\infty}} \leq 1 / 4$. This concludes the proof of Lemma 6.5 .

Proof of Proposition 6.4. To estimate $B_{\varepsilon}=-\operatorname{curl} H_{\varepsilon}$, we use

$$
B_{\varepsilon}(t, x)=-\operatorname{curl} v_{\varepsilon}(t, x, a)
$$

where

$$
v_{\varepsilon}=\nabla \phi_{\varepsilon}+H_{\varepsilon} .
$$

Let us consider the $k$-th component of $H(k=1,2,3)$ and set

$$
I=\int B_{\varepsilon k}(t, x) a(t, x) \mathrm{d} t \mathrm{~d} x .
$$

Thanks to Lemma 6.5, we have

$$
I=\iint B_{\varepsilon k}\left(t ; x_{1}+\theta W_{1} ; x_{2}+\sigma W_{2} ; x_{3}+\tau W_{3}\right) W_{1}\left(t, x_{1}\right) W_{2}\left(t, x_{2}\right) W_{3}\left(t, x_{3}\right) \mathrm{d} c \mathrm{~d} \theta \mathrm{d} \sigma \mathrm{d} \tau .
$$

To simplify notations, we deal with the case $k=1$. Since

$$
I=\iint\left(\partial_{3} v_{2}^{\varepsilon}-\partial_{2} v_{3}^{\varepsilon}\right)\left(t ; x_{1}+\theta W_{1} ; x_{2}+\sigma W_{2} ; x_{3}+\tau W_{3} ; a\right) W_{1}\left(t, x_{1}\right) W_{2}\left(t, x_{2}\right) W_{3}\left(t, x_{3}\right) \mathrm{d} c \mathrm{~d} \theta \mathrm{d} \sigma \mathrm{d} \tau
$$

we obtain, by using the mean value theorem

$$
\begin{aligned}
I= & \iint v_{2}^{\varepsilon}\left(t ; x_{1}+\theta W_{1} ; x_{2}+\sigma W_{2} ; x_{3}+W_{3} ; a\right) W_{1}\left(t, x_{1}\right) W_{2}\left(t, x_{2}\right) \mathrm{d} c \mathrm{~d} \theta \mathrm{d} \sigma \\
& -\iint v_{2}^{\varepsilon}\left(t ; x_{1}+\theta W_{1} ; x_{2}+\sigma W_{2} ; x_{3} ; a\right) W_{1}\left(t, x_{1}\right) W_{2}\left(t, x_{2}\right) \mathrm{d} c \mathrm{~d} \theta \mathrm{d} \sigma \\
& -\iint v_{3}^{\varepsilon}\left(t ; x_{1}+\theta W_{1} ; x_{2}+W_{2}\left(t, x_{2}\right) ; x_{3}+\tau W_{3} ; a\right) W_{1}\left(t, x_{1}\right) W_{3}\left(t, x_{3}\right) \mathrm{d} c \mathrm{~d} \theta \mathrm{d} \tau \\
& +\iint v_{3}^{\varepsilon}\left(t ; x_{1}+\theta W_{1} ; x_{2} ; x_{3}+\tau W_{3} ; a\right) W_{1}\left(t, x_{1}\right) W_{3}\left(t, x_{3}\right) \mathrm{d} c \mathrm{~d} \theta \mathrm{d} \tau .
\end{aligned}
$$


Then, by using Cauchy-Schwarz and by introducing $v$, we obtain

$$
\begin{aligned}
I^{2} \leq & C\left(\| W _ { 1 } \| _ { L ^ { \infty } } ^ { 2 } \| W _ { 2 } \| _ { L ^ { \infty } } ^ { 2 } \left[\iint\left|v_{2}^{\varepsilon}\left(t ; x_{1}+\theta W_{1} ; x_{2}+\sigma W_{2} ; x_{3}+W_{3} ; a\right)-v_{2}(t, x, a)\right|^{2} \mathrm{~d} c \mathrm{~d} \theta \mathrm{d} \sigma\right.\right. \\
& \left.+\iint\left|v_{2}^{\varepsilon}\left(t ; x_{1}+\theta W_{1} ; x_{2}+\sigma W_{2} ; x_{3} ; a\right)-v_{2}(t, x, a)\right|^{2} \mathrm{~d} c \mathrm{~d} \theta \mathrm{d} \sigma\right] \\
& +\left\|W_{1}\right\|_{L^{\infty}}^{2}\left\|W_{3}\right\|_{L^{\infty}}^{2}\left[\iint\left|v_{3}^{\varepsilon}\left(t ; x_{1}+\theta W_{1} ; x_{2}+W_{2} ; x_{3}+\tau W_{3} ; a\right)-v_{3}(t, x, a)\right|^{2} \mathrm{~d} c \mathrm{~d} \theta \mathrm{d} \tau\right. \\
& \left.\left.+\iint\left|v_{3}^{\varepsilon}\left(t ; x_{1}+\theta W_{1} ; x_{2} ; x_{3}+\tau W_{3} ; a\right)-v_{3}(t, x, a)\right|^{2} \mathrm{~d} c \mathrm{~d} \theta \mathrm{d} \tau\right]\right) .
\end{aligned}
$$

To complete the proof of Proposition 6.4 it is now enough to show the following lemma:

Lemma 6.6. Let $M$ be of form $M(t, x)=\tilde{M}(\zeta(t), x)=x+\tilde{W}(\zeta(t), x)$ with $\tilde{W}_{i}$ defined as in Lemma 6.5. Assume for $i=1,2,3, \quad l \leq m_{0}+1, \quad\left\|\partial_{i}^{l} \tilde{a}_{i}\right\|_{L^{\infty}} \leq \frac{1}{4}$. Then, we have the following estimate

$$
\frac{1}{2} \int\left|v_{\varepsilon}(t, M(t, x))-v(t, x)\right|^{2} \mathrm{~d} c \leq C\left(1+\sum_{i} \sum_{l=1}^{m_{0}}\left\|\partial_{t} \partial_{i}^{l} a_{i}\right\|_{L^{2}}^{2}\right)
$$

Remark 6.3. Subsequently, we omit parameters $\theta, \tau$ and $\sigma$ to simplify notations.

Proof of Lemma 6.6. For $A(t, x)=\prod a_{i}\left(t, x_{i}\right)$ where $a_{i}\left(t, x_{i}\right)=\zeta(t) \psi_{i}\left(x_{i}\right)$, we have to estimate

$$
\frac{1}{2} \int\left|v_{\varepsilon}(t, M(t, x))-v(t, x, a)\right|^{2} \mathrm{~d} c
$$

where $v_{\varepsilon}(t, x, a)=\nabla \phi_{\varepsilon}(t, x, a)+H_{\varepsilon}(t, x)$ and $M$ is defined by

$$
M(t, x)=\tilde{M}(\zeta(t), x)=x+W(t, x)=x+\tilde{W}(\zeta(t), x), \text { with } \tilde{W}_{i}\left(s, x_{i}\right)=\tilde{a}_{i}\left(s, x_{i}+W_{i}\left(t, x_{i}\right)\right),
$$

with $\left|\partial_{i} W_{i}\left(t, x_{i}\right)\right| \leq \frac{1}{3}$.

To estimate (32), we use

$$
\begin{aligned}
\frac{1}{2} \int\left|v_{\varepsilon}(t, M(t, x))-v(t, x, a)\right|^{2} \mathrm{~d} c \leq & \int\left|\left(\partial_{t}+v \cdot \nabla_{x}\right) M(t, x)-v_{\varepsilon}(t, M(t, x))\right|^{2} \mathrm{~d} c \\
& +\int\left|\left(\partial_{t}+v \cdot \nabla_{x}\right)(t, M(t, x))-v(t, x, a)\right|^{2} \mathrm{~d} c
\end{aligned}
$$

and observe that

$$
\frac{1}{2} \int\left|\left(\partial_{t}+v \cdot \nabla_{x}\right) M(t, x)-v(t, x, a)\right|^{2} \mathrm{~d} c \leq C\left(\int\left|\partial_{t}(M(t, x))\right|^{2} \mathrm{~d} t \mathrm{~d} x+\|\nabla M-I d\|_{L^{\infty}}^{2}\right) .
$$

Let us consider

$$
\frac{1}{2} \int\left|\left(\partial_{t}+v \cdot \nabla_{x}\right) M(t, x)-v_{\varepsilon}(t, M(t, x))\right|^{2} \mathrm{~d} c
$$


Thanks to Proposition 5.2, we obtain, as $u=0$,

$$
\begin{aligned}
& \int H_{\varepsilon}(t, M(t, x)) \partial_{t}(M(t, x)) \mathrm{d} t \mathrm{~d} x \\
& +\frac{1}{2} \int\left|\left(\partial_{t}+v \cdot \nabla_{x}\right) M(t, x)-v_{\varepsilon}(t, M(t, x))\right|^{2} \mathrm{~d} c \\
& \leq C\left(1+\int\left|\partial_{t} M(t, x)\right|^{2} \mathrm{~d} t \mathrm{~d} x+|| \nabla M \|_{L^{\infty}}^{2}\right) .
\end{aligned}
$$

Since $\left|\partial_{i} W_{i}\left(t, x_{i}\right)\right| \leq \frac{1}{3}, M$ is invertible and we can define $w$ and $\sigma$ so that

$$
\partial_{t} M(t, x)=\partial_{t}(\tilde{M}(\zeta(t), x))=\zeta^{\prime}(t) w(\zeta(t), \tilde{M}(\zeta(t), x))
$$

and $\partial_{t} \sigma+\nabla \cdot(\sigma w)=0$ with $\sigma(t=0, x)=1$.

We introduce

We have

$$
b(t, x)=\tilde{\beta}(\zeta(t), x), \quad \tilde{\beta}(s, x)=\int_{0}^{s} w(\theta, x) \sigma(\theta, x) \mathrm{d} \theta .
$$

$$
\begin{aligned}
& -\int H_{\varepsilon}(t, M(t, x)) \partial_{t} M(t, x) \mathrm{d} t \mathrm{~d} x \\
& =-\int H_{\varepsilon}(t, x) \zeta^{\prime}(t) w(\zeta(t), x) \sigma(\zeta(t), x) \mathrm{d} t \mathrm{~d} x \\
& =-\int H_{\varepsilon}(t, x) \partial_{t} b(t, x) \mathrm{d} t \mathrm{~d} x .
\end{aligned}
$$

The result of Part 6.1 implies that

$$
-\int H_{\varepsilon}(t, x) \partial_{t} b(t, x) \mathrm{d} t \mathrm{~d} x \leq C|| b \|_{G_{m_{0}}}
$$

for some integer $m_{0}$.

Then we obtain

$$
\begin{aligned}
\frac{1}{2} \int\left|\left(\partial_{t}+v \cdot \nabla_{x}\right) M(t, x)-v_{\varepsilon}(t, M(t, x))\right|^{2} \mathrm{~d} c \leq & C\left(1+\int\left|\partial_{t} M(t, x)\right|^{2}+\|\nabla M\|_{L^{\infty}}^{2}\right) \\
& -\int H_{\varepsilon}(t, x) \partial_{t} b(t, x) \mathrm{d} t \mathrm{~d} x \\
\leq & C\left(1+\int\left|\partial_{t} M(t, x)\right|^{2}+\|\nabla M\|_{L^{\infty}}^{2}+\|b\|_{G_{m}}\right) .
\end{aligned}
$$

To complete the Proof of 6.6 , it remains to estimate the right-hand side by some norms of the $a_{i}$.

\section{Comparison of the norms of $M$ and the $a_{i}$}

The quantities involved in the estimates are the following

$$
\int\left|\partial_{t}(M(t, x))\right|^{2} \mathrm{~d} t \mathrm{~d} x \text { and }\|\nabla M\|_{L^{\infty}}
$$


First, since $M(t, x)=x+W(t, x)=x+\tilde{W}(\zeta(t), x)$, the inequality $\left\|\partial_{i} W_{i}\right\|_{L^{\infty}} \leq \frac{1}{3}$ implies that $\|\nabla M\| L_{L^{\infty}} \leq C$. Moreover, to estimate $\int\left|\partial_{t} M(t, x)\right|^{2} \mathrm{~d} t \mathrm{~d} x$, we use the implicit definition (31) which can also be written as

$$
M_{i}\left(t, x_{i}\right)=x_{i}+a_{i}\left(t, M_{i}\left(t, x_{i}\right)\right)
$$

We obtain

Since $\left\|\partial_{i} a_{i}\right\|_{L^{\infty}} \leq$, we get

$$
\partial_{t} M_{i}\left(t, x_{i}\right)=\partial_{t} a_{i}\left(t, M_{i}\left(t, x_{i}\right)\right)+\partial_{t}\left(M_{i}\left(t, x_{i}\right)\right) \partial_{i} a_{i}\left(t, M_{i}\left(t, x_{i}\right)\right) .
$$

$$
\sum_{i}\left|\partial_{t} M_{i}\left(t, x_{i}\right)\right|^{2} \leq C \sum_{i}\left|\partial_{t} a_{i}\left(t, M_{i}\left(t, x_{i}\right)\right)\right|^{2}
$$

and

$$
\int\left|\partial_{t} M(t, x)\right|^{2} \mathrm{~d} t \mathrm{~d} x \leq C \sum_{i} \int\left|\partial_{t} a_{i}\left(t, x_{i}\right)\right|^{2} \mathrm{~d} x_{i}
$$

(using the change of variable $x_{i} \longrightarrow M_{i}\left(t, x_{i}\right)$ that just modifies $C$.)

\section{Comparison of the norms of $b$ and the $a_{i}$}

Let us denote $\||\cdot|\|$ the $L^{2}\left([0, T] ; H^{m_{0}}(D)\right)$ norm where $m_{0}$ is fixed large enough. We want to show that $\left\|\left|\partial_{t} b\right|\right\| \leq C||\left|\partial_{t} a\right| \| \mid$. Let us compare the norms of $b$ and the $a_{i}$. We have $b(t, x)=\tilde{b}(\zeta(t), x)$ where

$$
\tilde{b}(s, x)=\int_{0}^{s} w(\theta, x) \sigma(\theta, x) \mathrm{d} \theta .
$$

Here, $\sigma$ solves $\partial_{t} \sigma+\nabla \cdot(\sigma w)=0$, with $\sigma(t=0, x)=1$, and $w$ is implicitly defined by

$$
\partial_{s} \tilde{M}(s, x)=w(s, \tilde{M}(s, x)) .
$$

Let us recall that $\tilde{M}_{i}(s, x)=x_{i}+\tilde{W}_{i}\left(s, x_{i}\right)$ is linked to $\tilde{a}_{i}(s, x)=s \psi_{i}\left(x_{i}\right)$ through

$$
\tilde{W}_{i}\left(s, x_{i}\right)=\tilde{a}_{i}\left(x_{i}+\tilde{W}_{i}\left(s, x_{i}\right)\right) .
$$

Thus

$$
\partial_{i}^{l} \tilde{W}_{i}\left(s, x_{i}\right)=\partial_{i}^{l} \tilde{W}_{i}\left(s, x_{i}\right) \partial_{i} a_{i}\left(s, x_{i}+\tilde{W}_{i}\left(s, x_{i}\right)\right)+\text { rest }
$$

where the rest involves only derivatives of $\tilde{W}$ of order lower or equal to $(l-1)$ and derivatives of $\tilde{a}_{i}$ of order lower or equal to $l$.

So, the derivatives in $x_{i}$ of $\tilde{W}_{i}$ are controlled by those of $\tilde{a}_{i}$ and we get:

Lemma 6.7. Assume

$$
\left\|\partial_{i}^{l} \tilde{a}_{i}\right\|_{L^{\infty}} \leq C, \quad\left\|\partial_{i} \tilde{a}_{i}\right\|_{L^{\infty}} \leq \frac{1}{4}, \quad \forall l \leq m_{0} \quad \forall i
$$

Then, $x \rightarrow \tilde{M}(t, x)$ is uniformly smoothly invertible and for any $\alpha$ satisfying $|\alpha| \leq m_{0}$, for any $l \leq m_{0}$

$$
\begin{aligned}
& \left\|\partial_{i}^{l} \tilde{W}_{i}\right\|_{L^{\infty}} \leq C, \\
& \left\|\partial_{i}^{l} \tilde{M}_{i}\right\|_{L^{\infty}} \leq C,
\end{aligned}
$$




$$
\left\|\partial_{i}^{l} \tilde{M}^{-1}\right\|_{L^{\infty}} \leq C, \quad\left\|\partial^{\alpha} \sigma\right\|_{L^{\infty}} \leq C, \quad\left\|\partial^{\alpha} \frac{1}{\sigma}\right\|_{L^{\infty}} \leq C .
$$

Using the lemma, we get

$$
\sum_{|\alpha| \leq m_{0}} \int\left|\partial_{\alpha} \partial_{t} b\right|^{2} \mathrm{~d} t \mathrm{~d} x \leq C \sum_{l \leq m_{0}} \int \zeta^{\prime 2}(t)\left|\partial^{l} w(\zeta(t), x)\right|^{2} \mathrm{~d} t \mathrm{~d} x .
$$

In the same way, since $w(t, x)=\partial_{t} \tilde{M}\left(t, \tilde{M}^{-1}(t, x)\right)$, we obtain by differentiating this relation and using the bounds on the derivatives of $\tilde{M}^{-1}$ by their $L^{\infty}$ norm,

$$
\text { for every } l \leq m_{0},\left|\partial^{l} w(t, x)\right|^{2} \leq C \sum_{j \leq l}\left|\partial^{j} \partial_{t} \tilde{M}\left(t, \tilde{M}^{-1}(t, x)\right)\right|^{2} .
$$

And then

$$
\int \zeta^{\prime 2}(t)\left|\partial^{l} w(\zeta(t), x)\right|^{2} \mathrm{~d} t \mathrm{~d} x \leq C \sum_{j \leq l} \int \zeta^{\prime 2}(t)\left|\partial^{j} \partial_{t} \tilde{M}(\zeta(t), x)\right|^{2} \mathrm{~d} t \mathrm{~d} x
$$

since $\left\|\frac{1}{\sigma}\right\|_{L^{\infty}} \leq C$.

Thus, we have $\left|\left\|\partial_{t} b\right\|\right||\leq C|\left\|\partial_{t} M \mid\right\|$. Next, since $M(t, x)=x+a(t, M(t, x))$ we obtain

$$
\begin{aligned}
\left\|\partial_{t} M\right\| & =\left\|\partial_{t}(a(t, M(t, \cdot)))\right\| \mid \\
& \leq C\left(\left\||| \partial_{t} a\right\|\left|+\left\|\partial_{x} a(t, M) \partial_{t} M\right\|\right|\right)
\end{aligned}
$$

(using Leibnitz formula and Lem. 6.7). Finally, using the smallness of the $\left\|\partial_{i}^{l} \tilde{a}_{i}\right\|_{L^{\infty}}$ we get

$$
\left\|\partial _ { t } M \left|\left\||\leq C||| \partial_{t} a \mid\right\|\right.\right.
$$

and therefore $\left|\left\|\partial_{t} b\right\|\right| \mid$ is controlled by $\left|\left\|\partial_{t} a \mid\right\|\right.$. This concludes the proof of Proposition 6.4.

Second case: General test functions

Proposition 6.8. There are constants $C, r_{0}>0$ and an integer $m_{1}$ such that for any test function $h(t, x)$ compactly supported in $0<t<T$ with a norm in $G_{m_{1}}=H^{1, m_{1}}([0, T] \times(D))$ smaller than $r_{0}>0$,

$$
\left|\int B_{\varepsilon}(t, x) h(t, x) \mathrm{d} t \mathrm{~d} x\right| \leq C .
$$

Proof. As in the proof for the time derivative, we write the test function as a Fourier series. We obtain

We have

$$
\int B_{\varepsilon}(t ; x) h(t, x) \mathrm{d} t \mathrm{~d} x=\sum_{k \in \mathbb{Z}^{3}} \int B_{\varepsilon}(t ; x) \hat{h}(t, k) \mathrm{e}^{2 i \pi k \cdot x} \mathrm{~d} t \mathrm{~d} x .
$$

$$
\begin{aligned}
\int B_{\varepsilon}(t ; x) h(t, x) \mathrm{d} t \mathrm{~d} x= & \sum_{k \in \mathbb{Z}^{3}} \int B_{\varepsilon}(t ; x) \frac{1}{\langle k\rangle^{2}}\left(\langle k\rangle^{2} \theta^{3}(t) \hat{h}(t, k)+\theta^{3}(t) L(k)\right) \mathrm{e}^{2 i \pi k \cdot x} \mathrm{~d} t \mathrm{~d} x \\
& -\sum_{k \in \mathbb{Z}^{3}} \int B_{\varepsilon}(t ; x) \frac{1}{\langle k\rangle^{2}} \theta^{3}(t) L(k) \mathrm{e}^{2 i \pi k \cdot x} \mathrm{~d} t \mathrm{~d} x
\end{aligned}
$$

where $\langle k\rangle=\sqrt{1+|k|^{2}}, \theta \in C_{c}^{\infty}(] 0, T[;[0,1])$ is chosen so that $\theta(t)=1$ on the support of $h$ and

$$
L(k)=C\left(2\langle k\rangle^{-N}+i 2\langle k\rangle^{-N}\right)
$$


with $N$ to be chosen later. Both $\theta$ and $L(k)$ have been introduced because we need to extract a cubic root without producing spurious singularities.

To estimate this expression, we just have to consider two typical terms

$$
I_{1}=\sum_{k \in \mathbb{Z}^{3}} \int B_{\varepsilon}(t ; x) \frac{1}{\langle k\rangle^{2}} \theta^{3}(t)\left(\langle k\rangle^{2} \operatorname{Re}(\hat{h}(t, k))+2\langle k\rangle^{-N}\right) \cos \left(2 \pi k_{1} x_{1}\right) \cos \left(2 \pi k_{2} x_{2}\right) \cos \left(2 \pi k_{3} x_{3}\right) \mathrm{d} t \mathrm{~d} x
$$

and

$$
I_{2}=\sum_{k \in \mathbb{Z}^{3}} \int 2 B_{\varepsilon}(t ; x) \frac{1}{\langle k\rangle^{2}}\langle k\rangle^{-N} \theta^{3}(t) \cos \left(2 \pi k_{1} x_{1}\right) \cos \left(2 \pi k_{2} x_{2}\right) \cos \left(2 \pi k_{3} x_{3}\right) \mathrm{d} t \mathrm{~d} x
$$

Let us first consider $I_{2}$.

$$
I_{2}=2 \sum_{k \in \mathbb{Z}^{3}} \int B_{\varepsilon}(t ; x) \frac{1}{\langle k\rangle^{2}}\langle k\rangle^{-N} \prod_{j} a_{j}\left(t, x_{j}, k_{j}\right) \mathrm{d} t \mathrm{~d} x
$$

with $a_{j}\left(t, x_{j}, k_{j}\right)=\theta(t) \cos \left(2 \pi k_{j} x_{j}\right)$. Using Proposition 6.4 , we easily get

$$
\left|I_{2}\right| \leq \sum_{k \in \mathbb{Z}^{3}} C \frac{\langle k\rangle^{m_{0}}}{\langle k\rangle^{2}}\langle k\rangle^{-N} \leq C
$$

for $N$ large enough (the constant depending only on the support of $h$ through the choice of $\theta$ ).

Let us next consider $I_{1}$.

$$
I_{1}=\sum_{k \in \mathbb{Z}^{3}} \int B_{\varepsilon}(t ; x) \frac{1}{\langle k\rangle^{2}} \prod_{j} a_{j}\left(t, x_{j}, k_{j}\right) \mathrm{d} t \mathrm{~d} x
$$

with

$$
a_{j}\left(t, x_{j}, k_{j}\right)=\zeta(t) \psi_{i}\left(x_{i}\right)
$$

where

$$
\zeta(t)=\langle k\rangle^{m} \theta(t) \sqrt[3]{\langle k\rangle^{2} \operatorname{Re}(\hat{h}(t, k))+2\langle k\rangle^{-N}}, \quad \psi_{i}\left(x_{i}\right)=\langle k\rangle^{-m} \cos \left(2 \pi k_{j} x_{j}\right),
$$

where $m$ will be chosen later. Let us first get a pointwise decay estimate on $\hat{h}(t, k)$. We have

$$
\begin{aligned}
\langle k\rangle^{m_{1}} \sup _{t}|\hat{h}(t, k)| & \leq\left(\int\left|\langle k\rangle^{m_{1}} \hat{\partial_{t}} h(t, k)\right|^{2} \mathrm{~d} t\right)^{\frac{1}{2}} \\
& \leq C\left(\sum_{|\alpha| \leq m_{1}} \iint\left|\partial^{\alpha} \partial_{t} h(t, x)\right|^{2} \mathrm{~d} x \mathrm{~d} t\right)^{\frac{1}{2}} \leq C
\end{aligned}
$$


by assumption. Thus $\sup _{t}|\hat{h}(t, k)| \leq\langle k\rangle^{-m_{1}}$. So, we can choose $m_{1}, N, m$ and $r_{0}$ in such a way that $\zeta(t)$ and the $\psi_{i}\left(x_{i}\right)$ satisfy the assumption of Proposition 6.4 and we finally get

$$
\left|I_{1}\right| \leq C \sum_{k \in \mathbb{Z}^{3}}\left|\langle k\rangle^{m_{1}} \partial_{t} \hat{h}(t, k)\right|^{2} \mathrm{~d} t
$$

which can be bounded in terms of $\|h\|_{G_{m_{1}}}$.

\section{Approximate estimates for the Velocity GRAdients}

We prove inequality (13) which formally means that the $L^{2}\left(Q^{\prime}, \mathrm{d} c\right)$ norm of the gradient in space of $v$ is bounded.

Thanks to (11) we just have to show

$$
\int_{Q^{\prime}}\left|\left(\nabla_{x} \phi_{\varepsilon}+H_{\varepsilon}\right) \circ \mathrm{e}^{\delta \zeta(t) w}-v\right|^{2} \mathrm{~d} c \leq C\left(\varepsilon^{2}+\delta^{2}+\delta \chi(\varepsilon)\right),
$$

for every smooth divergence free vector field $w(x)$, taking $\zeta \in C_{c}^{\infty} \infty(] 0, T[;[0,1])$ so that $\zeta(t)=1$ for $\tau \leq t \leq T-\tau$.

Since the transformations $\mathrm{e}^{\delta \zeta(t) w}$ can be written

$$
\mathrm{e}^{\delta \zeta(t) w}=x+\delta \zeta(t) w(x)+\delta^{2} g(t, x)
$$

with $g=g_{\delta}$ smooth and uniformly bounded in $\delta$, we have

$$
\int_{Q^{\prime}}\left|\left(\partial_{t}+v \cdot \nabla_{x}\right) \mathrm{e}^{\delta \zeta(t) w}-v(t, x, a)\right|^{2} \mathrm{~d} c=O\left(\delta^{2}\right)
$$

since

$$
\begin{aligned}
\int_{Q^{\prime}}\left|\left(\partial_{t}+v \cdot \nabla_{x}\right) \mathrm{e}^{\delta \zeta(t) w}-v(t, x, a)\right|^{2} \mathrm{~d} c= & \int_{Q^{\prime}} \mid \delta \zeta^{\prime}(t) w(x)+\delta^{2} \partial_{t} g(t, x)+\delta \zeta(t) v(t, x, a) \cdot \nabla w(x) \\
& +\left.\delta^{2} v(t, x, a) \cdot \nabla g(t, x)\right|^{2} \mathrm{~d} c
\end{aligned}
$$

with $v \in L^{2}\left(Q^{\prime}, \mathrm{d} c\right)$. Now, it remains to estimate

$$
\frac{1}{2} \int_{Q^{\prime}}\left|\left(\partial_{t}+v \cdot \nabla_{x}\right) \mathrm{e}^{\delta \zeta(t) w}-\left(\nabla_{x} \phi_{\varepsilon}+H_{\varepsilon}\right) \circ \mathrm{e}^{\delta \zeta(t) w}\right|^{2} \mathrm{~d} c .
$$


For that purpose, we consider inequality (19) when $\eta=0$, and we expand its right-hand side term in powers of $\delta$ :

$$
\begin{aligned}
& \frac{1}{2} \int_{Q^{\prime}}\left|\left(\partial_{t}+v \cdot \nabla_{x}\right) \mathrm{e}^{\delta \zeta(t) w}\right|^{2} \mathrm{~d} c-\frac{1}{2} \int_{Q^{\prime}}|v(t, x, a)|^{2} \mathrm{~d} c \\
= & \frac{1}{2} \int_{Q^{\prime}}\left|\left(\delta \zeta^{\prime}(t) w(x)+v(t, x, a)+\delta \zeta(t) v(t, x, a) \cdot \nabla w(x)\right)+\delta^{2} \partial_{t} g(t, x)+\delta^{2} v(t, x, a) \cdot \nabla g(t, x)\right|^{2} \mathrm{~d} c \\
& -\frac{1}{2} \int_{Q^{\prime}}|v(t, x, a)|^{2} \mathrm{~d} c \\
= & \frac{1}{2} \int_{Q^{\prime}} \delta^{2}\left(\zeta^{\prime}(t)\right)^{2}|w(x)|^{2}+\frac{1}{2} \int_{Q^{\prime}}|v(t, x, a)|^{2} \\
& +\frac{1}{2} \int_{Q^{\prime}} \delta^{2}(\zeta(t))^{2}|v(t, x, a) \cdot \nabla w(x)|^{2}+\int_{Q^{\prime}} \delta^{2} \zeta^{\prime}(t) \zeta(t) w(x) \cdot(v(t, x, a) \cdot \nabla w(x)) \\
& +\int_{Q^{\prime}} \delta \zeta^{\prime}(t) w(x) \cdot v(t, x, a)+\int_{Q^{\prime}} \delta \zeta(t) v(t, x, a) \cdot(v(t, x, a) \cdot \nabla w(x)) \mathrm{d} c \\
& +O\left(\delta^{2}\right)-\frac{1}{2} \int_{Q^{\prime}}|v(t, x, a)|^{2} \mathrm{~d} c .
\end{aligned}
$$

The terms of order 0 can be simplified and those involving $g$ are bounded by $C\left(\delta^{2}\right)$. Moreover

$$
\begin{aligned}
& \int_{Q^{\prime}} \delta^{2}\left(\zeta^{\prime}(t)\right)^{2}|w(x)|^{2}+\int_{Q^{\prime}} \delta^{2} \zeta^{\prime}(t) \zeta(t) w \cdot(v(t, x, a) \cdot \nabla w(x))+\int_{Q^{\prime}} \delta^{2} \zeta(t)^{2}|v(t, x, a)|^{2}|\nabla w(x)|^{2} \\
= & \int_{Q^{\prime}} \delta^{2}\left(\zeta^{\prime}(t)\right)^{2}|w(x)|^{2}+\int_{Q^{\prime}} \delta^{2} \zeta^{\prime}(t) \zeta(t) w(x) \cdot(u(t, x) \cdot \nabla w(x))+\int_{Q^{\prime}} \delta^{2} \zeta(t)^{2}|v(t, x, a)|^{2}|\nabla w(x)|^{2} \\
= & O\left(\delta^{2}\right)
\end{aligned}
$$

since the bound on energy enables us to estimate $\int_{Q^{\prime}}|v|^{2}(t, x, a)|\nabla w(x)|^{2} \mathrm{~d} c$.

We have shown that

$$
\frac{1}{2} \int_{Q^{\prime}}\left|\left(\partial_{t}+v \cdot \nabla_{x}\right) \mathrm{e}^{\delta \zeta(t) w}\right|^{2} \mathrm{~d} c-\frac{1}{2} \int_{Q^{\prime}}|v|^{2} \mathrm{~d} c=\Lambda \delta+O\left(\delta^{2}\right)
$$

with

$$
\Lambda=\int_{Q^{\prime}}\left(\zeta^{\prime}(t) w(x) \cdot v(t, x, a)+\zeta(t) v(t, x, a) \cdot(v(t, x, a) \cdot \nabla w(x))\right) \mathrm{d} c
$$

Let us expand $\int_{Q^{\prime}} H_{\varepsilon} \cdot\left(\mathrm{d} m^{\delta}-\mathrm{d} m\right)$ in the same way.

$$
\int_{Q^{\prime}} H_{\varepsilon} \cdot\left(\mathrm{d} m^{\delta}-\mathrm{d} m\right)=\int_{Q^{\prime}} H_{\varepsilon} \circ \mathrm{e}^{\delta \zeta(t) w}\left[\left(\partial_{t}+v \cdot \nabla_{x}\right) \mathrm{e}^{\delta \zeta(t) w}\right] \mathrm{d} c-\int_{Q^{\prime}} H_{\varepsilon} \cdot v \mathrm{~d} c .
$$


Since $w$ is divergence free, the transformation $\mathrm{e}^{\delta \zeta(t) w}$ is Lebesgue measure-preserving and then we have (denoting by $H_{\varepsilon, i}$ the $i^{\text {th }}$ component of $H_{\varepsilon}$ )

$$
\begin{aligned}
\int_{Q^{\prime}} H_{\varepsilon} \cdot\left(\mathrm{d} m^{\delta}-\mathrm{d} m\right)= & \int_{Q^{\prime}} H_{\varepsilon, i}(t, x)\left[\delta \zeta^{\prime}(t) w_{i}(x-\delta \zeta(t) w(x))+v_{i}(t, x-\delta \zeta(t) w(x), a)\right. \\
& +\delta \zeta(t) v_{j}(t, x-\delta \zeta(t) w(x), a) \partial_{j} w_{i}(x-\delta \zeta(t) w(x)) \\
& \left.+\delta^{2} \partial_{t} g_{i}(t, x-\delta \zeta(t) w(x))+\delta^{2} v_{j}(t, x-\delta \zeta(t) w(x), a) \partial_{j} g_{i}(t, x-\delta \zeta(t) w(x))\right] \mathrm{d} c \\
& -\int_{Q^{\prime}} H_{\varepsilon, i}(t, x) v_{i}(t, x, a) \mathrm{d} c .
\end{aligned}
$$

Integrating with respect to $a$, we obtain

$$
\begin{aligned}
\int_{Q^{\prime}} H_{\varepsilon} \cdot\left(\mathrm{d} m^{\delta}-\mathrm{d} m\right)= & \int_{Q} H_{\varepsilon, i}(t, x)\left[\delta \zeta^{\prime}(t) w_{i}(x-\delta \zeta(t) w(x))+u_{i}(t, x-\delta \zeta(t) w(x)\right. \\
& +\delta \zeta(t) u_{j}(t, x-\delta \zeta(t) w(x)) \partial_{j} w_{i}(x-\delta \zeta(t) w(x)) \\
& \left.+\delta^{2} \partial_{t} g_{i}(t, x-\delta \zeta(t) w(x))+\delta^{2} u_{j}(t, x-\delta \zeta(t) w(x)) \partial_{j} g_{i}(t, x-\delta \zeta(t) w(x))\right] \mathrm{d} t \mathrm{~d} x \\
& -\int_{Q} H_{\varepsilon, i}(t, x) u_{i}(t, x) \mathrm{d} t \mathrm{~d} x
\end{aligned}
$$

The following equalities

$$
\begin{aligned}
& w_{i}(x-\delta \zeta(t) w(x))=w_{i}(x)+\delta g_{i}^{1}(t, x) \\
& u_{i}(t, x-\delta \zeta(t) w(x))=u_{i}(t, x)-\delta \zeta(t) w_{j}(x) \partial_{j} u_{i}(t, x)+\delta^{2} g_{i}^{2}(t, x)
\end{aligned}
$$

where $g^{1}=g_{\delta}^{1}$ and $g^{2}=g_{\delta}^{2}$ are smooth and uniformly bounded in $\delta$, ensure

$$
\begin{aligned}
\int_{Q^{\prime}} H_{\varepsilon} \cdot\left(\mathrm{d} m^{\delta}-\mathrm{d} m\right)= & \delta \int_{Q} H_{\varepsilon, i}\left[\zeta^{\prime}(t) w_{i}(x)-\zeta(t) w_{j}(x) \partial_{j} u_{i}(t, x)+\zeta(t) u_{j}(t, x) \partial_{j} w_{i}(x)\right] \mathrm{d} t \mathrm{~d} x \\
& +\int_{Q} H_{\varepsilon, i} \delta^{2} g_{i}^{3}(t, x)
\end{aligned}
$$

where $g^{3}=g_{\delta}^{3}$ is smooth and uniformly bounded in $\delta$. Since $H_{\varepsilon}$ is bounded in $\mathcal{D}^{\prime}(Q)$, we can write

$$
\int_{Q^{\prime}} H_{\varepsilon} \cdot\left(\mathrm{d} m^{\delta}-\mathrm{d} m\right)=\Lambda_{\varepsilon} \delta+O\left(\delta^{2}\right)
$$

with

$$
\Lambda_{\varepsilon}=\delta \int_{Q} H_{\varepsilon, i}\left[\zeta^{\prime}(t) w_{i}(x)-\zeta(t) w_{j}(x) \partial_{j} u_{i}(t, x)+\zeta(t) u_{j}(t, x) \partial_{j} w_{i}(x)\right] \mathrm{d} t \mathrm{~d} x
$$


The sequence $\Lambda_{\varepsilon}$ has a limit when $\varepsilon \rightarrow 0$ (up to a subsequence) since $H^{\varepsilon}$ is bounded in $\mathcal{D}^{\prime}(Q)$. Then, equation (19) becomes

$$
\delta \Lambda_{\varepsilon}-\delta \Lambda \leq \varepsilon^{2}+O\left(\delta^{2}\right)
$$

where

$$
\Lambda=\int_{Q^{\prime}}\left(\zeta^{\prime}(t) w(x) \cdot v(t, x, a)+\zeta(t) v(t, x, a) \cdot(v(t, x, a) \cdot \nabla w(x))\right) \mathrm{d} c
$$

When $\varepsilon \rightarrow 0$, and then $\delta \rightarrow 0$, we obtain

$$
\lim _{\varepsilon \rightarrow 0} \Lambda_{\varepsilon}=\Lambda
$$

Then, introducing $\chi(\varepsilon)=\max \left(\Lambda_{\varepsilon}-\Lambda ; \varepsilon\right) \stackrel{\varepsilon \rightarrow 0}{\longrightarrow} 0$, we have

$$
\begin{aligned}
\mid \int_{Q^{\prime}}\left(\zeta^{\prime}(t) w_{i}(x) v_{i}(t, x, a)+\right. & \left.\zeta(t) v_{i}(t, x, a) v_{j}(t, x, a) \partial_{j} w_{i}(x)\right) \mathrm{d} c \\
& -\int_{Q} H_{\varepsilon, i}(t, x) \zeta^{\prime}(t) w_{i}(x)-\int \zeta(t) w_{i}(x) u_{j}(t, x)\left(\partial_{i} H_{\varepsilon, j}-\partial_{j} H_{\varepsilon, i}\right) \mathrm{d} t \mathrm{~d} x \mid \leq \chi(\varepsilon) .
\end{aligned}
$$

This estimate will be useful later and can be written

$$
\begin{aligned}
\mid-\int_{Q}\left(\zeta(t) w(x) \cdot \partial_{t} u(t, x)+\zeta(t) w(x)\right. & \left.\operatorname{div} \int(v(t, x, a) \otimes v(t, x, a)) c(t, x, \mathrm{~d} a)\right) \mathrm{d} t \mathrm{~d} x \\
& +\int_{Q} \partial_{t} H_{\varepsilon}(t, x) \cdot \zeta(t) w(x)-\zeta(t) w(x) \cdot\left(u(t, x) \wedge \operatorname{curl} H_{\varepsilon}(t, x)\right) \mathrm{d} t \mathrm{~d} x \mid \leq \chi(\varepsilon) .
\end{aligned}
$$

Remark 7.1. As $\varepsilon \rightarrow 0$, we see that

$$
\partial_{t} u+\operatorname{div} \int(v \otimes v) c(t, x, \mathrm{~d} a)-\partial_{t} H+u \wedge \operatorname{curl} H
$$

is a gradient in the sense that it is orthogonal to the space of all divergence free vector fields. So we obtain the optimality equation integrated in $a$ and projected onto the space of all divergence free vector fields.

We use inequality

$$
\left(\Lambda_{\varepsilon}-\Lambda\right) \geq-\chi(\varepsilon)
$$

to express (19) as

$$
\begin{aligned}
& \frac{1}{2} \int_{Q^{\prime}}\left|\left(\partial_{t}+v \cdot \nabla_{x}\right) \mathrm{e}^{\delta \zeta(t) w}-\left(\nabla_{x} \phi_{\varepsilon}+H_{\varepsilon}\right) \circ \mathrm{e}^{\delta \zeta(t) w}\right|^{2} \mathrm{~d} c-\delta \chi(\varepsilon)+O\left(\delta^{2}\right) \\
& \leq \frac{1}{2} \int_{Q^{\prime}}\left|\left(\partial_{t}+v \cdot \nabla_{x}\right) \mathrm{e}^{\delta \zeta(t) w}-\left(\nabla_{x} \phi_{\varepsilon}+H_{\varepsilon}\right) \circ \mathrm{e}^{\delta \zeta(t) w}\right|^{2} \mathrm{~d} c+\delta\left(\Lambda_{\varepsilon}-\Lambda\right)+O\left(\delta^{2}\right) \leq \varepsilon^{2}+O\left(\delta^{2}\right) .
\end{aligned}
$$


If we choose $\zeta \in C_{c}^{\infty}(] 0, T[;[0,1])$ so that

$$
\begin{aligned}
& \zeta(t)=0 \text { if } 0 \leq t \leq \frac{\tau}{2} \text { or } T-\frac{\tau}{2} \leq t \leq T \\
& \zeta(t)=1 \text { if } \tau \leq t \leq T-\tau
\end{aligned}
$$

we can bound

$$
\int_{Q_{\tau}}\left|\left(\partial_{t}+v \cdot \nabla_{x}\right) \mathrm{e}^{\delta w}-\left(\nabla_{x} \phi_{\varepsilon}+H_{\varepsilon}\right) \circ \mathrm{e}^{\delta w}\right|^{2} \mathrm{~d} c
$$

by

$$
\int_{Q^{\prime}}\left|\left(\partial_{t}+v \cdot \nabla_{x}\right) \mathrm{e}^{\delta \zeta(t) w}-\left(\nabla_{x} \phi_{\varepsilon}+H_{\varepsilon}\right) \circ \mathrm{e}^{\delta \zeta(t) w}\right|^{2} \mathrm{~d} c
$$

and (38) leads to (13).

\section{A first SET OF APPROXimate optimality EQUATIONS}

Here, we show Proposition 2.4 starting from

$$
\frac{1}{\delta} \int_{Q^{\prime}}\left[\partial_{t} \phi_{\varepsilon}+\frac{1}{2}\left|\left(\nabla_{x} \phi_{\varepsilon}+H_{\varepsilon}\right)\right|^{2}(t, x+\delta \omega, a)-\partial_{t} \phi_{\varepsilon}-\frac{1}{2}\left|\left(\nabla_{x} \phi_{\varepsilon}+H_{\varepsilon}\right)\right|^{2}(t, x, a)\right] f(t, x, a) \mathrm{d} c \leq \frac{\varepsilon^{2}}{\delta},
$$

which comes from Proposition 2.1. In this expression, $f(t, x, a)$ is a continuous function with values in [0,1], compactly supported in $0<t<T$ with continuous partial derivatives and $\omega$ is a fixed vector in $\mathbb{R}^{d}$.

We split the left-hand side into two integral terms:

$$
I_{1}=\frac{1}{\delta} \int_{Q^{\prime}}\left[\partial_{t} \phi_{\varepsilon}(t, x+\delta \omega, a)-\partial_{t} \phi_{\varepsilon}(t, x, a)\right] f(t, x, a) \mathrm{d} c
$$

and

$$
I_{2}=\frac{1}{2 \delta} \int_{Q^{\prime}}\left[\left|\left(\nabla_{x} \phi_{\varepsilon}+H_{\varepsilon}\right)(t, x+\delta \omega, a)\right|^{2}-\left|\left(\nabla_{x} \phi_{\varepsilon}+H_{\varepsilon}\right)(t, x, a)\right|^{2}\right] f(t, x, a) \mathrm{d} c .
$$

Let us study the first integral term. Thanks to the mean value theorem, it becomes

$$
I_{1}=\int_{Q^{\prime}} \int_{0}^{1}\left[\partial_{t} \nabla_{x} \phi_{\varepsilon}(t, x+\sigma \delta \omega, a) \cdot \omega\right] f(t, x, a) \mathrm{d} c \mathrm{~d} \sigma .
$$

Then, equation (6) enables us to write

$$
\begin{aligned}
I_{1}= & -\int_{Q^{\prime}} \int_{0}^{1} \partial_{t} f(t, x, a) \nabla_{x} \phi_{\varepsilon}(t, x+\sigma \delta \omega, a) \cdot \omega \mathrm{d} c \mathrm{~d} \sigma \\
& \left.-\int_{Q^{\prime}} \int_{0}^{1}\left(v(t, x, a) \cdot \nabla_{x} f(t, x, a)\right) \nabla_{x} \phi_{\varepsilon}(t, x+\sigma \delta \omega, a)\right) \cdot \omega \mathrm{d} c \mathrm{~d} \sigma \\
& -\int_{Q^{\prime}} \int_{0}^{1} v(t, x, a) \cdot \nabla_{x}\left[\nabla_{x} \phi_{\varepsilon}(t, x+\sigma \delta \omega, a) \cdot \omega\right] f(t, x, a) \mathrm{d} c \mathrm{~d} \sigma .
\end{aligned}
$$


Introducing $H_{\varepsilon}$ and $v_{\varepsilon}=\nabla \phi_{\varepsilon}+H_{\varepsilon}$, we obtain

$$
\begin{aligned}
I_{1}= & -\int_{Q^{\prime}} \int_{0}^{1} \partial_{t} f(t, x, a)\left(\nabla_{x} \phi_{\varepsilon}+H_{\varepsilon}\right)(t, x+\sigma \delta \omega, a) \cdot \omega \mathrm{d} c \mathrm{~d} \sigma \\
& -\int_{Q^{\prime}} \int_{0}^{1}\left(v(t, x, a) \cdot \nabla_{x} f(t, x, a)\right)\left(\nabla_{x} \phi_{\varepsilon}+H_{\varepsilon}\right)(t, x+\sigma \delta \omega, a) \cdot \omega \mathrm{d} c \mathrm{~d} \sigma \\
& -\frac{1}{\delta} \int_{Q^{\prime}} v(t, x, a) \cdot\left[\left(\nabla_{x} \phi_{\varepsilon}+H_{\varepsilon}\right)(t, x+\delta \omega, a)-\left(\nabla_{x} \phi_{\varepsilon}+H_{\varepsilon}\right)(t, x, a)\right] f(t, x, a) \mathrm{d} c \\
& +\int_{Q^{\prime}} \int_{0}^{1} \partial_{t} f(t, x, a) H_{\varepsilon}(t, x+\sigma \delta \omega) \cdot \omega \mathrm{d} c \mathrm{~d} \sigma \\
& +\int_{Q^{\prime}} \int_{0}^{1}\left(v(t, x, a) \cdot \nabla_{x} f(t, x, a)\right) H_{\varepsilon}(t, x+\sigma \delta \omega) \cdot \omega \mathrm{d} c \mathrm{~d} \sigma \\
& +\int_{Q^{\prime}} \int_{0}^{1} v(t, x, a) \cdot\left[\omega \cdot \nabla_{x} H_{\varepsilon}(t, x+\sigma \delta \omega)\right] f(t, x, a) \mathrm{d} c \mathrm{~d} \sigma \\
= & I_{1}^{1}+I_{1}^{2}+I_{1}^{3}+I_{1}^{4}+I_{1}^{5}+I_{1}^{6} .
\end{aligned}
$$

Since we notice that

$$
\begin{aligned}
I_{1}^{6}= & \int_{Q^{\prime}} \int_{0}^{1} v(t, x, a) \cdot\left[\left(\omega \cdot \nabla_{x}\right) H_{\varepsilon}(t, x+\sigma \delta \omega)\right] f(t, x, a) \mathrm{d} c \mathrm{~d} \sigma \\
= & \int_{Q^{\prime}} \int_{0}^{1}\left(v(t, x, a) \cdot \nabla_{x}\right)\left[H_{\varepsilon}(t, x+\sigma \delta \omega) \cdot \omega\right] f(t, x, a) \mathrm{d} c \mathrm{~d} \sigma \\
& +\int_{Q^{\prime}} \int_{0}^{1}\left[v(t, x, a) \wedge \operatorname{curl} H_{\varepsilon}(t, x+\sigma \delta \omega)\right] \cdot \omega f(t, x, a) \mathrm{d} c \mathrm{~d} \sigma
\end{aligned}
$$

we obtain

$$
\begin{aligned}
I_{1}^{4}+I_{1}^{5}+I_{1}^{6}= & \int_{Q^{\prime}} \int_{0}^{1}-\partial_{t} H_{\varepsilon}(t, x+\sigma \delta \omega) \cdot \omega f(t, x, a) \mathrm{d} c \mathrm{~d} \sigma \\
& +\int_{Q^{\prime}} \int_{0}^{1}\left[v(t, x, a) \wedge \operatorname{curl} H_{\varepsilon}(t, x+\sigma \delta \omega)\right] \cdot \omega f(t, x, a) \mathrm{d} c \mathrm{~d} \sigma
\end{aligned}
$$

So using (13), it comes

$$
\begin{aligned}
I_{1}^{1}+I_{1}^{2}= & -\int_{Q^{\prime}} \partial_{t} f(t, x, a) v(t, x, a) \cdot \omega \mathrm{d} c-\int_{Q^{\prime}}\left[v(t, x, a) \cdot \nabla_{x} f(t, x, a) v(t, x, a)\right] \cdot \omega \mathrm{d} c \\
& +O_{f}\left(\sqrt{\delta^{2}+\delta \chi(\varepsilon)+\varepsilon^{2}}\right)=O_{f}(\delta+\chi(\varepsilon)+\varepsilon)
\end{aligned}
$$


(where $O_{f}$ means $O$ with constants involving $f$ ) and then

$$
\begin{aligned}
I_{1} \geq & -\int_{Q^{\prime}} \partial_{t} f(t, x, a) v(t, x, a) \cdot \omega \mathrm{d} c-\int_{Q^{\prime}}\left[v(t, x, a) \cdot \nabla_{x} f(t, x, a) v(t, x, a)\right] \cdot \omega \mathrm{d} c \\
& -\frac{1}{\delta} \int_{Q^{\prime}} v(t, x, a) \cdot\left[\left(\nabla_{x} \phi_{\varepsilon}+H_{\varepsilon}\right)(t, x+\delta \omega, a)-\left(\nabla_{x} \phi_{\varepsilon}+H_{\varepsilon}\right)(t, x, a)\right] f(t, x, a) \mathrm{d} c \\
& \int_{Q^{\prime}} \int_{0}^{1}-\partial_{t} H_{\varepsilon}(t, x+\sigma \delta \omega) \cdot \omega f(t, x, a) \mathrm{d} c \mathrm{~d} \sigma \\
& +\int_{Q^{\prime}} \int_{0}^{1}\left[v(t, x, a) \wedge \operatorname{curl} H_{\varepsilon}(t, x+\sigma \delta \omega)\right] \cdot \omega f(t, x, a) \mathrm{d} c \mathrm{~d} \sigma \\
& -C_{f}\left(\delta^{2}+\delta \chi(\varepsilon)+\varepsilon^{2}\right)^{\frac{1}{2}} .
\end{aligned}
$$

The integral term $I_{2}$ can be estimated thanks to (13). Indeed,

$$
\begin{aligned}
I_{2} & =\frac{1}{2 \delta} \int_{Q^{\prime}}\left[\left|\left(\nabla_{x} \phi_{\varepsilon}+H_{\varepsilon}\right)(t, x+\delta \omega, a)\right|^{2}-\left|\left(\nabla_{x} \phi_{\varepsilon}+H_{\varepsilon}\right)(t, x, a)\right|^{2}\right] f \mathrm{~d} c \\
& =\frac{1}{2 \delta} \int_{Q^{\prime}}\left[\left(\nabla_{x} \phi_{\varepsilon}+H_{\varepsilon}\right)(t, x+\delta \omega, a)-\left(\nabla_{x} \phi_{\varepsilon}+H_{\varepsilon}\right)(t, x, a)\right] \cdot \\
& =\left[\left(\nabla_{x} \phi_{\varepsilon}+H_{\varepsilon}\right)(t, x+\delta \omega, a)+\left(\nabla_{x} \phi_{\varepsilon}+H_{\varepsilon}\right)(t, x, a)\right] f \mathrm{~d} c \\
& \geq \frac{1}{\delta} \int_{Q^{\prime}}\left[\left(\nabla_{x} \phi_{\varepsilon}+H_{\varepsilon}\right)(t, x+\delta \omega, a)-\left(\nabla_{x} \phi_{\varepsilon}+H_{\varepsilon}\right)(t, x, a)\right] \cdot v f \mathrm{~d} c-\frac{1}{\delta}\left(\delta^{2}+\varepsilon^{2}+\delta \chi(\varepsilon)\right) C_{f} .
\end{aligned}
$$

So, inequality $I_{1}+I_{2} \leq \frac{1}{\delta} \varepsilon^{2}$ implies

$$
\begin{aligned}
& \int_{Q^{\prime}} \int_{0}^{1}-\partial_{t} H_{\varepsilon}(t, x+\sigma \delta \omega) \cdot \omega f(t, x, a) \mathrm{d} c \mathrm{~d} \sigma+\int_{Q^{\prime}} \int_{0}^{1}\left[v(t, x, a) \wedge \operatorname{curl} H_{\varepsilon}(t, x+\sigma \delta \omega)\right] \cdot \omega f(t, x, a) \mathrm{d} c \mathrm{~d} \sigma \\
& \quad-\int_{Q^{\prime}} \partial_{t} f(t, x, a) v(t, x, a) \cdot \omega \mathrm{d} c-\int_{Q^{\prime}} v(t, x, a) \cdot \nabla_{x} f(t, x, a) v(t, x, a) \cdot \omega \mathrm{d} c \\
& \leq C_{f} \frac{1}{\delta}\left(\delta^{2}+\varepsilon^{2}+\delta \chi(\varepsilon)\right)
\end{aligned}
$$


since $\left(\delta^{2}+\varepsilon^{2}+\delta \chi(\varepsilon)\right)^{\frac{1}{2}} \leq \frac{1}{\delta}\left(\delta^{2}+\varepsilon^{2}+\delta \chi(\varepsilon)\right)$. Let $\xi(t)$ be a test function in [0,1], compactly supported in time and equal to 1 on the support of $f$, we apply (39) to $(1-f) \xi=\xi-f$. We have

$$
\begin{aligned}
& \int_{Q^{\prime}} \int_{0}^{1}-\partial_{t} H_{\varepsilon}(t, x+\sigma \delta \omega) \cdot(-\omega f)(t, x, a) \mathrm{d} c \mathrm{~d} \sigma \\
& +\int_{Q^{\prime}} \int_{0}^{1}\left[v(t, x, a) \wedge \operatorname{curl} H_{\varepsilon}(t, x+\sigma \delta \omega)\right] \cdot(-\omega f)(t, x, a) \mathrm{d} c \mathrm{~d} \sigma \\
& -\int_{Q^{\prime}} \partial_{t}(-f)(t, x, a) v(t, x, a) \cdot \omega \mathrm{d} c-\int_{Q^{\prime}}\left[v(t, x, a) \cdot \nabla_{x}(-f)(t, x, a) v(t, x, a)\right] \cdot \omega \mathrm{d} c \\
& +\int_{Q} \int_{0}^{1}-\partial_{t} H_{\varepsilon}(t, x+\sigma \delta \omega) \cdot \omega \xi(t) \mathrm{d} t \mathrm{~d} x \mathrm{~d} \sigma+\int_{Q} \int_{0}^{1}\left[u(t, x) \wedge \operatorname{curl} H_{\varepsilon}(t, x+\sigma \delta \omega)\right] \cdot \omega \xi(t) \mathrm{d} t \mathrm{~d} x \mathrm{~d} \sigma \\
& -\int_{Q} \partial_{t} \xi(t) u(t, x) \cdot \omega \mathrm{d} t \mathrm{~d} x-\int_{Q^{\prime}} v(t, x, a) \cdot \nabla_{x} \xi(t) v(t, x, a) \cdot \omega \mathrm{d} c \leq C_{f} \frac{1}{\delta}\left(\delta^{2}+\varepsilon^{2}+\delta \chi(\varepsilon)\right)
\end{aligned}
$$

that can be written

$$
\begin{aligned}
& \int_{Q^{\prime}} \int_{0}^{1}-\partial_{t} H_{\varepsilon}(t, x+\sigma \delta \omega) \cdot(-\omega f)(t, x, a) \mathrm{d} c \mathrm{~d} \sigma \\
& +\int_{Q^{\prime}} \int_{0}^{1}\left[v(t, x, a) \wedge \operatorname{curl} H_{\varepsilon}(t, x+\sigma \delta \omega)\right] \cdot(-\omega f)(t, x, a) \mathrm{d} c \mathrm{~d} \sigma \\
& +\int_{Q^{\prime}} \partial_{t} f(t, x, a) v(t, x, a) \cdot \omega \mathrm{d} c+\int_{Q^{\prime}}\left[v(t, x, a) \cdot \nabla_{x} f(t, x, a) v(t, x, a)\right] \cdot \omega \mathrm{d} c \\
& +\int_{Q}-\partial_{t} H_{\varepsilon}(t, y) \cdot \omega \xi(t) \mathrm{d} t \mathrm{~d} y+\int_{Q} \int_{0}^{1}\left[u(t, y-\sigma \delta \omega) \wedge \operatorname{curl} H_{\varepsilon}(t, y)\right] \cdot \omega \xi(t) \mathrm{d} t \mathrm{~d} y \mathrm{~d} \sigma \\
& -\int_{Q} \xi^{\prime}(t) u(t, x) \cdot \omega \mathrm{d} t \mathrm{~d} x \leq C_{f} \frac{1}{\delta}\left(\delta^{2}+\varepsilon^{2}+\delta \chi(\varepsilon)\right) .
\end{aligned}
$$


Since $H_{\varepsilon}$ is bounded in $\mathcal{D}^{\prime}(Q)$, we can write

$$
\begin{aligned}
& \int_{Q^{\prime}} \int_{0}^{1}-\partial_{t} H_{\varepsilon}(t, x+\sigma \delta \omega) \cdot \omega(-f)(t, x, a) \mathrm{d} c \mathrm{~d} \sigma \\
& +\int_{Q^{\prime}} \int_{0}^{1}\left[v(t, x, a) \wedge \operatorname{curl} H_{\varepsilon}(t, x+\sigma \delta \omega)\right] \cdot \omega(-f)(t, x, a) \mathrm{d} c \mathrm{~d} \sigma \\
& -\int_{Q^{\prime}} \partial_{t}(-f)(t, x, a) v(t, x, a) \cdot \omega \mathrm{d} c-\int_{Q^{\prime}}\left[v(t, x, a) \cdot \nabla_{x}(-f)(t, x, a) v(t, x, a)\right] \cdot \omega \mathrm{d} c \\
& +\int_{Q}-\partial_{t} H_{\varepsilon}(t, x) \cdot \omega \xi(t) \mathrm{d} t \mathrm{~d} x+\int_{Q}\left[u(t, x) \wedge \operatorname{curl} H_{\varepsilon}(t, x)\right] \cdot \omega \xi(t) \mathrm{d} t \mathrm{~d} x \\
& -\int_{Q} \xi^{\prime}(t) u(t, x) \cdot \omega \mathrm{d} t \mathrm{~d} x \leq C_{f} \frac{1}{\delta}\left(\delta^{2}+\varepsilon^{2}+\delta \chi(\varepsilon)\right) .
\end{aligned}
$$

Then, using (37), we simplify the three last terms of the left-hand side. Finally, we obtain for any test function with values in $[0,1]$

$$
\begin{aligned}
& \mid \int_{Q^{\prime}} \int_{0}^{1}-\partial_{t} H_{\varepsilon}(t, x+\sigma \delta \omega) \cdot \omega f(t, x, a) \mathrm{d} c \mathrm{~d} \sigma \\
& +\int_{Q^{\prime}} \int_{0}^{1}\left[v(t, x, a) \wedge \operatorname{curl} H_{\varepsilon}(t, x+\sigma \delta \omega)\right] \cdot \omega f(t, x, a) \mathrm{d} c \mathrm{~d} \sigma \\
& -\int_{Q^{\prime}} \partial_{t} f(t, x, a) v(t, x, a) \cdot \omega \mathrm{d} c-\int_{Q^{\prime}}\left[v(t, x, a) \cdot \nabla_{x} f(t, x, a) v(t, x, a)\right] \cdot \omega \mathrm{d} c \mid \leq C_{f} \frac{1}{\delta}\left(\delta^{2}+\varepsilon^{2}+\delta \chi(\varepsilon)\right) .
\end{aligned}
$$

This concludes the proof of Proposition 2.4.

Let us notice that we also have

$$
\begin{aligned}
& I_{1}+I_{2} \geq \int_{Q^{\prime}} \int_{0}^{1}-\partial_{t} H_{\varepsilon}(t, x+\sigma \delta \omega) \cdot \omega f(t, x, a) \mathrm{d} c \mathrm{~d} \sigma \\
& +\int_{Q^{\prime}} \int_{0}^{1}\left[v(t, x, a) \wedge \operatorname{curl} H_{\varepsilon}(t, x+\sigma \delta \omega)\right] \cdot \omega f(t, x, a) \mathrm{d} c \mathrm{~d} \sigma \\
& -\int_{Q^{\prime}} \partial_{t} f(t, x, a) v(t, x, a) \cdot \omega \mathrm{d} c-\int_{Q^{\prime}}\left[v(t, x, a) \cdot \nabla_{x} f(t, x, a) v(t, x, a)\right] \cdot \omega \mathrm{d} c \\
& -C_{f}\left(\frac{1}{\delta}\left(\delta^{2}+\varepsilon^{2}+\delta \chi(\varepsilon)\right)\right)=O_{f}\left(\frac{1}{\delta}\left(\delta^{2}+\varepsilon^{2}+\delta \chi(\varepsilon)\right)\right) .
\end{aligned}
$$

\section{Mollified APPROXIMATE OPTIMALITY EQUATIONS}

Let us consider the quantities studied in the previous section

$$
I_{1}=\frac{1}{\delta} \int_{Q^{\prime}}\left[\partial_{t} \phi_{\varepsilon}(t, x+\delta \omega, a)-\partial_{t} \phi_{\varepsilon}(t, x, a)\right] f(t, x, a) \mathrm{d} c
$$


and

$$
I_{2}=\frac{1}{2 \delta} \int_{Q^{\prime}}\left[\left|\left(\nabla_{x} \phi_{\varepsilon}+H_{\varepsilon}\right)(t, x+\delta \omega, a)\right|^{2}-\left|\left(\nabla_{x} \phi_{\varepsilon}+H_{\varepsilon}\right)(t, x, a)\right|^{2}\right] f(t, x, a) \mathrm{d} c .
$$

We recall that $I_{1}+I_{2} \leq \frac{\varepsilon^{2}}{\delta}$. Let us rewrite this inequality for $-\omega$ and $\xi(1-f)$, where $\xi$ is a test function depending only on time and equal to 1 on the support of $f$.

Adding the two inequalities, we obtain

$$
I_{1}^{\prime}+I_{2}^{\prime}+I_{3}^{\prime} \leq 2 \frac{\varepsilon^{2}}{\delta}
$$

with

$$
\begin{gathered}
I_{1}^{\prime}=\frac{1}{\delta} \int_{Q^{\prime}}\left[\partial_{t} \phi_{\varepsilon}(t, x+\delta \omega, a)-\partial_{t} \phi_{\varepsilon}(t, x-\delta \omega, a)\right] f(t, x, a) \mathrm{d} c \\
I_{2}^{\prime}=\frac{1}{2 \delta} \int_{Q^{\prime}}\left[\left|\left(\nabla_{x} \phi_{\varepsilon}+H_{\varepsilon}\right)(t, x+\delta \omega, a)\right|^{2}-\left|\left(\nabla_{x} \phi_{\varepsilon}+H_{\varepsilon}\right)(t, x-\delta \omega, a)\right|^{2}\right] f(t, x, a) \mathrm{d} c
\end{gathered}
$$

and

$$
\begin{aligned}
I_{3}^{\prime}= & \frac{1}{\delta} \int_{Q^{\prime}}\left[\partial_{t} \phi_{\varepsilon}(t, x+\delta \omega, a)-\partial_{t} \phi_{\varepsilon}(t, x, a)\right] \xi(t) \mathrm{d} c \\
& +\frac{1}{2 \delta} \int_{Q^{\prime}}\left[\left|\left(\nabla_{x} \phi_{\varepsilon}+H_{\varepsilon}\right)(t, x+\delta \omega, a)\right|^{2}-\left|\left(\nabla_{x} \phi_{\varepsilon}+H_{\varepsilon}\right)(t, x, a)\right|^{2}\right] \xi(t) \mathrm{d} c .
\end{aligned}
$$

But, thanks to (41) (applied to $f(t, x, a)=\xi(t)$ ), we have

$$
\begin{aligned}
I_{3}^{\prime} \geq & \int_{Q} \int_{0}^{1}-\partial_{t} H_{\varepsilon}(t, x+\sigma \delta \omega) \cdot \omega \xi(t) \mathrm{d} t \mathrm{~d} x \mathrm{~d} \sigma \\
& +\int_{Q} \int_{0}^{1}\left[u(t, x) \wedge \operatorname{curl} H_{\varepsilon}(t, x+\sigma \delta \omega)\right] \cdot \omega \xi(t) \mathrm{d} t \mathrm{~d} x \mathrm{~d} \sigma \\
& -\int_{Q} \partial_{t} \xi(t) u(t, x) \cdot \omega \mathrm{d} t \mathrm{~d} x-\int_{Q^{\prime}} v(t, x, a) \cdot \nabla_{x} \xi(t) v(t, x, a) \cdot \omega \mathrm{d} c-C \frac{1}{\delta}\left(\delta^{2}+\varepsilon^{2}+\delta \chi(\varepsilon)\right) .
\end{aligned}
$$

Which becomes, since $H_{\varepsilon}$ is bounded in $\mathcal{D}^{\prime}(Q)$,

$$
\begin{aligned}
I_{3}^{\prime} \geq & \int_{Q}-\partial_{t} H_{\varepsilon}(t, x) \cdot \omega \xi(t) \mathrm{d} t \mathrm{~d} x+\int_{Q}\left[u(t, x) \wedge \operatorname{curl} H_{\varepsilon}(t, x)\right] \cdot \omega \xi(t) \mathrm{d} t \mathrm{~d} x \\
& -\int_{Q} \partial_{t} \xi(t) u(t, x) \cdot \omega \mathrm{d} t \mathrm{~d} x-\int_{Q^{\prime}} v(t, x, a) \cdot \nabla_{x} \xi(t) v(t, x, a) \cdot \omega \mathrm{d} c-C \frac{1}{\delta}\left(\delta^{2}+\varepsilon^{2}+\delta \chi(\varepsilon)\right)
\end{aligned}
$$

and then, thanks to (37), we obtain

then finally

$$
I_{3}^{\prime} \geq-C \frac{1}{\delta}\left(\delta^{2}+\varepsilon^{2}+\delta \chi(\varepsilon)\right)
$$

$$
\begin{aligned}
\frac{1}{\delta} \int_{Q^{\prime}}\left[\partial_{t} \phi_{\varepsilon}(t, x+\delta \omega, a)-\partial_{t} \phi_{\varepsilon}(t, x-\delta \omega, a)\right] & f(t, x, a) \mathrm{d} c+\frac{1}{2 \delta} \int_{Q^{\prime}}\left[\left|\left(\nabla_{x} \phi_{\varepsilon}+H_{\varepsilon}\right)(t, x+\delta \omega, a)\right|^{2}\right. \\
& \left.-\left|\left(\nabla_{x} \phi_{\varepsilon}+H_{\varepsilon}\right)(t, x-\delta \omega, a)\right|^{2}\right] f(t, x, a) \mathrm{d} c \leq C \frac{1}{\delta}\left(\delta^{2}+\varepsilon^{2}+\delta \chi(\varepsilon)\right) .
\end{aligned}
$$


In order to smoothen this expression, we perform the change of variable $\omega \rightarrow \omega+y$ for every $y$, we multiply by a (radial) mollifier $\gamma$ defined on $\mathbb{R}^{d}$ and we integrate with respect to $y$. We obtain

$$
\begin{array}{rr}
\frac{1}{\delta} \iint_{Q^{\prime}}\left[\partial_{t} \phi_{\varepsilon}(t, x+\delta \omega+\delta y, a)-\partial_{t} \phi_{\varepsilon}(t, x-\delta \omega-\delta y, a)\right] f(t, x, a) \gamma(y) \mathrm{d} c \mathrm{~d} y & \\
+\frac{1}{2 \delta} \iint_{Q^{\prime}}\left[\left|\left(\nabla_{x} \phi_{\varepsilon}+H_{\varepsilon}\right)(t, x+\delta \omega+\delta y, a)\right|^{2}-\left|\left(\nabla_{x} \phi_{\varepsilon}+H_{\varepsilon}\right)(t, x-\delta \omega-\delta y, a)\right|^{2}\right] f(t, x, a) \gamma(y) \mathrm{d} c \mathrm{~d} y & \leq C \frac{1}{\delta}\left(\delta^{2}+\varepsilon^{2}+\delta \chi(\varepsilon)\right) .
\end{array}
$$

Assuming $\gamma$ to be radial, we have $\gamma(-y)=\gamma(y)$, and then

$$
\begin{array}{rr}
\frac{1}{\delta} \iint_{Q^{\prime}}\left[\partial_{t} \phi_{\varepsilon}(t, x+\delta y+\delta \omega, a)-\partial_{t} \phi_{\varepsilon}(t, x+\delta y-\delta \omega, a)\right] f(t, x, a) \gamma(y) \mathrm{d} c \mathrm{~d} y & \\
+\frac{1}{2 \delta} \iint_{Q^{\prime}}\left[\left|\left(\nabla_{x} \phi_{\varepsilon}+H_{\varepsilon}\right)(t, x+\delta y+\delta \omega, a)\right|^{2}-\left|\left(\nabla_{x} \phi_{\varepsilon}+H_{\varepsilon}\right)(t, x+\delta y-\delta \omega, a)\right|^{2}\right] f(t, x, a) \gamma(y) \mathrm{d} c \mathrm{~d} y & \leq C \frac{1}{\delta}\left(\delta^{2}+\varepsilon^{2}+\delta \chi(\varepsilon)\right) .
\end{array}
$$

Then, we notice that

$$
\begin{aligned}
\frac{1}{\delta} \iint_{Q^{\prime}}\left[\partial_{t} \phi_{\varepsilon}(t, x+\delta y+\delta \omega, a)-\partial_{t} \phi_{\varepsilon}(t, x+\right. & \delta y-\delta \omega, a)] f(t, x, a) \gamma(y) \mathrm{d} c \mathrm{~d} y \\
& =\frac{1}{\delta} \int_{-1}^{1} \iint_{Q^{\prime}}\left[\partial_{t} \nabla \phi_{\varepsilon}(t, x+\delta y+\sigma \delta \omega, a) \cdot \omega\right] f(t, x, a) \gamma(y) \mathrm{d} c \mathrm{~d} \sigma \mathrm{d} y
\end{aligned}
$$

Remark 9.1. The parity of $\gamma$ enables us to uncouple $\sigma$ and $y$ and then to preserve the smoothness provided by the convolution.

The same computations as in the previous part lead to the following mollified approximate optimality equations:

$$
\begin{aligned}
& \mid-\int_{Q^{\prime}} \partial_{t} f(t, x, a) v(t, x, a) \cdot \omega \mathrm{d} c-\int_{Q^{\prime}} v(t, x, a) \cdot \nabla_{x} f(t, x, a) v(t, x, a) \cdot \omega \mathrm{d} c \\
& \iint_{Q^{\prime}} \int_{-1}^{1}-\partial_{t} H_{\varepsilon}(t, x+\delta y+\sigma \delta \omega) \cdot \omega f(t, x, a) \gamma(y) \mathrm{d} c \mathrm{~d} \sigma \mathrm{d} y \\
& +\iint_{Q^{\prime}} \int_{-1}^{1}\left[v(t, x, a) \wedge \operatorname{curl} H_{\varepsilon}(t, x+\delta y+\sigma \delta \omega)\right] \cdot \omega f(t, x, a) \gamma(y) \mathrm{d} c \mathrm{~d} \sigma \mathrm{d} y \mid \\
& \leq C_{f} \frac{1}{\delta}\left(\delta^{2}+\varepsilon^{2}+\delta \chi(\varepsilon)\right) .
\end{aligned}
$$


When $f$ does not depend on $a$, inequality (40) involves only smooth quantities and then, we can pass to the limit as $\varepsilon \rightarrow 0$ and then $\delta \rightarrow 0$. We obtain

$$
\partial_{t} u+\nabla \cdot \int_{a} c v \otimes v-\partial_{t} H+u \wedge \operatorname{curl} H=0
$$

This equation is nothing but the integral in $a$ of the desired optimality equation (10).

\section{Estimates For the time DeRIVATives}

Let us prove Proposition 2.5. First, we show inequality (14), using (19) when $\delta=0$ (no spatial deformation),

$$
\begin{aligned}
& \int_{Q} H_{\varepsilon}(t, x) u(t+\eta \zeta(t), x)\left(1+\eta \zeta^{\prime}(t)\right) \mathrm{d} t \mathrm{~d} x \\
& -\int_{Q} H_{\varepsilon}(t, x) u(t, x) \mathrm{d} t \mathrm{~d} x+\frac{1}{2} \int_{Q^{\prime}}\left|v^{\eta}-\left(\nabla_{x} \phi_{\varepsilon}+H_{\varepsilon}\right)\right|^{2} \mathrm{~d} c^{\eta} \\
& \leq \varepsilon^{2}+\frac{1}{2} \int_{Q^{\prime}}\left|v^{\eta}\right|^{2} \mathrm{~d} c^{\eta}-\frac{1}{2} \int_{Q^{\prime}}|v|^{2} \mathrm{~d} c,
\end{aligned}
$$

with transformations satisfying

$$
t^{\prime}=t+\eta \zeta(t) \text { and } \tau_{\eta}\left(t^{\prime}\right)=t=t^{\prime}+O(\eta)
$$

We recall that $\eta$ can be chosen small enough so that $t \rightarrow t+\eta \zeta(t)$ is a diffeomorphism from $[0, T]$ to $[0, T]$.

Let us first simplify the following quantity

$$
\begin{aligned}
I= & \int_{Q^{\prime}}\left|v^{\eta}-\left(\nabla_{x} \phi_{\varepsilon}+H_{\varepsilon}\right)\right|^{2} \mathrm{~d} c^{\eta} \\
\leq & \varepsilon^{2}-\int_{Q} H_{\varepsilon}(t, x) u(t+\eta \zeta(t), x)\left(1+\eta \zeta^{\prime}(t)\right) \mathrm{d} t \mathrm{~d} x+\int_{Q} H_{\varepsilon}(t, x) u(t, x) \mathrm{d} t \mathrm{~d} x \\
& +\frac{1}{2} \int_{Q^{\prime}}|v(t+\eta \zeta(t), x, a)|^{2}\left(1+\eta \zeta^{\prime}(t)\right) \mathrm{d} c^{\eta} \\
& -\frac{1}{2} \int_{Q^{\prime}}|v(t, x, a)|^{2} \mathrm{~d} c \\
& +\frac{1}{2} \eta \int_{Q^{\prime}} \zeta^{\prime}(t)|v(t+\eta \zeta(t), x, a)|^{2}\left(1+\eta \zeta^{\prime}(t)\right) \mathrm{d} c^{\eta} \\
= & \varepsilon^{2}+I_{1}+I_{2}+I_{3}+I_{4} .
\end{aligned}
$$

We have

$$
\begin{gathered}
I_{1}=-\eta \int \zeta^{\prime}(t)\left(\int H_{\varepsilon}(t, x) u(t, x) \mathrm{d} x\right) \mathrm{d} t-\eta \int \zeta(t)\left[\int H_{\varepsilon}(t, x) \partial_{t} u(t, x) \mathrm{d} x\right] \mathrm{d} t+O\left(\eta^{2}\right) \\
I_{2}=-I_{3}
\end{gathered}
$$


(obtained using the change of variable $t \rightarrow t+\eta \zeta(t)$ ) and

$$
\begin{aligned}
I_{4} & =\frac{1}{2} \eta \int \zeta^{\prime}\left(\tau_{\eta}(t)\right)\left[\int|v(t, x, a)|^{2} c(t, x, \mathrm{~d} a) \mathrm{d} x\right] \mathrm{d} t \\
& =\eta \int \zeta^{\prime}(t)\left(\int \frac{1}{2}|v(t, x, a)|^{2} c(t, x, \mathrm{~d} a) \mathrm{d} x\right) \mathrm{d} t+O\left(\eta^{2}\right) .
\end{aligned}
$$

So we have

$$
\begin{aligned}
\int_{Q^{\prime}} \mid v(t+\eta \zeta(t), x, a)-\left(\nabla_{x} \phi_{\varepsilon}-H_{\varepsilon}\right)( & t, x, a)\left.\right|^{2} \mathrm{~d} c^{\eta} \leq \varepsilon^{2}-\eta \int \zeta^{\prime}(t)\left[\int \frac{1}{2}|v(t, x, a)|^{2} c(t, x, \mathrm{~d} a) \mathrm{d} x\right. \\
& \left.+\int H_{\varepsilon}(t, x) u(t, x) \mathrm{d} x\right] \mathrm{d} t-\eta \int \zeta(t)\left[\int H_{\varepsilon}(t, x) \partial_{t} u(t, x) \mathrm{d} x\right] \mathrm{d} t+O\left(\eta^{2}\right) .
\end{aligned}
$$

This inequality can be written

$$
\int_{Q^{\prime}}\left|v(t+\eta \zeta(t), x, a)-\left(\nabla_{x} \phi_{\varepsilon}+H_{\varepsilon}\right)(t, x, a)\right|^{2} \mathrm{~d} c^{\eta} \leq \varepsilon^{2}-\eta \Lambda_{\varepsilon}+O\left(\eta^{2}\right)
$$

where

tends to

$$
\Lambda_{\epsilon}=\int \zeta^{\prime}(t) \int \frac{1}{2}|v(t, x, a)|^{2} c(t, x, \mathrm{~d} a) \mathrm{d} x+\int H_{\varepsilon}(t, x) \partial_{t}(\zeta(t) u(t, x)) \mathrm{d} x \mathrm{~d} t
$$

Then

$$
\lim \Lambda_{\epsilon}=\int \zeta^{\prime}(t) \int \frac{1}{2}|v(t, x, a)|^{2} c(t, x, \mathrm{~d} a) \mathrm{d} x-\left\langle\partial_{t} H(t, x), \zeta(t) u(t, x)\right\rangle .
$$

$$
\int_{Q^{\prime}}\left|v(t+\eta \zeta(t), x, a)-\left(\nabla_{x} \phi_{\varepsilon}+H_{\varepsilon}\right)(t, x, a)\right|^{2} \mathrm{~d} c^{\eta} \leq \varepsilon^{2}+\eta \chi^{\prime}(\varepsilon)+O\left(\eta^{2}\right)
$$

with $\chi^{\prime}(\varepsilon)=\max \left(\left|\Lambda_{\varepsilon}\right|, \varepsilon\right)$. Let us notice that $\chi^{\prime}(\varepsilon) \geq \varepsilon$ and $\chi^{\prime}(\varepsilon) \stackrel{\varepsilon \rightarrow 0}{\longrightarrow} 0$.

Indeed, (43) implies that

$$
\eta . \lim _{\varepsilon \rightarrow 0} \Lambda_{\varepsilon} \leq C \eta^{2}
$$

then

and then

$$
\lim _{\varepsilon \rightarrow 0} \Lambda_{\varepsilon}=0
$$

$$
\forall \zeta \in C_{c}^{\infty}(] 0, T[), \quad\left\langle-\partial_{t} H(t, x) ; \zeta(t) u(t, x)\right\rangle+\left\langle\zeta^{\prime}(t) ; \frac{1}{2} \int|v(t, x, a)|^{2} c(t, x, \mathrm{~d} a)\right\rangle=0 .
$$

Finally, if we choose $\zeta \geq 0$ so that $\zeta=1$ on $Q_{\tau}$, we obtain (14).

Remark 10.1. In the case where $u=0,(44)$ implies the conservation of the kinetic energy. In the case where $u$ is not zero, (44) implies that the kinetic energy belongs to $L^{\infty}([0, T])$. Indeed, using the optimality equation integrated in $a(42)$, we get

$$
\partial_{t} \frac{1}{2} \int|v(t, x, a)|^{2} c(t, x, \mathrm{~d} a) \mathrm{d} x=\int\left[\partial_{t} u_{i}(t, x) u_{i}(t, x)+\partial_{j} u_{i}(t, x) \int v_{i}(t, x, a) v_{j}(t, x, a) c(t, x, \mathrm{~d} a)\right] \mathrm{d} x .
$$


Denoting $f(t)=\int|v(t, x, a)|^{2} c(t, x, \mathrm{~d} a) \mathrm{d} x,(45)$ implies that there exists $C_{0}$ so that:

$$
\partial_{t} f(t)-C_{0} f(t) \leq C
$$

Since we know that

$$
\int_{0}^{T} f(t) \mathrm{d} t \leq C
$$

because $(c, m)$ has finite Action, there is at least $t_{0}$ such that $f\left(t_{0}\right) \leq C$. Using the Gronwall lemma, we obtain

$$
f(t) \leq C \mathrm{e}^{C_{0} t}
$$

The kinetic energy is then bounded for every time $t \in[0, T]$.

\section{Derivation of MOLlified optimality EQUATIONS}

We want now to pass to the limit when $\varepsilon \rightarrow 0$ in the mollified approximate optimality equation (9), using that

$$
\left\langle\partial_{t} H_{\varepsilon} ; g\right\rangle \rightarrow\left\langle\partial_{t} H ; g\right\rangle
$$

and

$$
\left\langle\operatorname{curl} H_{\varepsilon} ; g\right\rangle \rightarrow\langle\operatorname{curl} H ; g\rangle
$$

for all function $g(t, x)$, compactly supported in $0<t<T$ with a finite norm in $H^{1, N}([0, T] \times D)$, for $N$ large enough. Then we get mollified optimality equations.

\subsection{Terms involving $\operatorname{curl} H_{\varepsilon}$}

In this section, we justify the limit for the term of (9) involving $\operatorname{curl} H_{\varepsilon}$

$$
\iint_{Q^{\prime}} \int_{-1}^{1}\left[v(t, x, a) \wedge \operatorname{curl} H_{\varepsilon}(t, x+\delta y+\sigma \delta \omega+y)\right] \cdot \omega f(t, x, a) \gamma(y) \mathrm{d} c \mathrm{~d} \sigma \mathrm{d} y .
$$

This term can be written

$$
\int g(t, x) \wedge \operatorname{curl} H_{\varepsilon}(t, x) \cdot \omega \mathrm{d} t \mathrm{~d} x
$$

where $g=g_{\delta, \omega, \gamma}$ is defined by

$$
g(t, x)=\int_{-1}^{1} \int f(t, x-\delta y-\sigma \delta \omega, a) v(t, x-\delta y-\sigma \delta \omega, a) c(t, x-\delta y-\sigma \delta \omega, \mathrm{d} a) \gamma(y) \mathrm{d} y \mathrm{~d} \sigma .
$$

Remark 11.1. In those computations, we use the following abusive notation

$$
\int f(t, x, a) c(t, x-\delta y-\sigma \delta \omega, a) \mathrm{d} a
$$


instead of

$$
\int f(t, x, a) c(t, x-\delta y-\sigma \delta \omega, \mathrm{d} a)
$$

and we abusively write integral signs instead of duality brackets.

Remark 11.2. The parameter $\delta>0$ will remain fixed and we use the notations $\mathrm{d}_{\gamma} y=\gamma(y) \mathrm{d} y$ and

$$
T_{\sigma, \delta}(y)=\delta y+\sigma \delta \omega .
$$

Remark 11.3. In some estimates, we use that the following functions belong to $L^{\infty}(Q)$,

$$
C(t, x)=\int c\left(t, x-T_{\sigma, \delta}(y), a\right) \gamma(y) \mathrm{d} y \mathrm{~d} \sigma \mathrm{d} a,
$$

that in fact is equal to 1 ,

which is equal to

$$
V(t, x)=\int c v^{2}\left(t, x-T_{\sigma, \delta}(y), a\right) \gamma(y) \mathrm{d} y \mathrm{~d} \sigma \mathrm{d} a,
$$

with

$$
\int_{D \times A} c v^{2}(t, z, a) \gamma_{\mathbb{T}^{d}}(z-x+\sigma \delta \omega) \mathrm{d} z \mathrm{~d} a
$$

$$
\gamma_{\mathbb{T}^{d}}(y)=\sum_{k \in \mathbb{Z}^{d}} \delta^{-d} \gamma((y-k) / \delta)
$$

and then belongs to $L^{\infty}$ since the kinetic energy is bounded.

We have to show that $g$ has a finite $H^{1, N}([0, T] \times D)$ norm for $N$ large enough and is compactly supported in $0<t<T$. Notice first that since $f(t, x, a)$ is compactly supported in $0<t<T$, so is $g(t, x)$. The mollification by $\gamma$ ensures that $g(t, x)$ is smooth in $x \in D$.

It remains to show that $\partial_{t} g$ belongs to $L^{2}(Q)$ with $Q=[0, T] \times D$ since the convolution enables us to transfer the derivatives in space on $\gamma$.

We show the following proposition

Proposition 11.1. For every function $p(t, x) \in L^{2}(Q)$, we have

$$
\left\langle p(t, x) ; \partial_{t} g(t, x)\right\rangle \leq C\|p\|_{L^{2}(Q)} .
$$

Remark 11.4. Formally, we write

$$
\begin{aligned}
\partial_{t} g & =\int \partial_{t} f v c+f v \partial_{t} c+f \partial_{t} v c \\
& =\int \partial_{t} f v c-\nabla(f v) \cdot v c+f \partial_{t} v c
\end{aligned}
$$

Then using the formal limit of the estimates obtained for the derivatives of the velocity $v$, we obtain a $L^{2}$ bound for $\partial_{t} g$.

Proof. For every $p \in C_{c}^{\infty}(] 0, T[\times D)$, we write

$$
\begin{aligned}
\left\langle p(t, x) ; \partial_{t} g(t, x)\right\rangle & =\lim _{\eta \rightarrow 0} \int_{Q} p(t, x) \frac{[g(t+\eta, x)-g(t, x)]}{\eta} \mathrm{d} t \mathrm{~d} x \\
& =\lim _{\eta \rightarrow 0} I_{1}+I_{2}
\end{aligned}
$$


with $T_{\sigma, \delta}(y)=\delta y+\sigma \delta \omega$. Since the function $p$ is a smooth test function, there exists $\tau$ so that

$$
\int_{Q} p(t, x) \frac{[g(t+\eta, x)-g(t, x)]}{\eta} \mathrm{d} t \mathrm{~d} x=\int_{Q_{\tau}} p(t, x) \frac{[g(t+\eta, x)-g(t, x)]}{\eta} \mathrm{d} t \mathrm{~d} x
$$

let us split this integral formula in two terms. The first one is

$$
\begin{aligned}
I_{1} & =\int_{Q_{\tau}} p(t, x)\left(\int \frac{\left[f\left(t+\eta, x-T_{\sigma, \delta}(y), a\right)-f\left(t, x-T_{\sigma, \delta}(y), a\right)\right]}{\eta} c v\left(t, x-T_{\sigma, \delta}(y), a\right) \gamma(y) \mathrm{d} y \mathrm{~d} \sigma \mathrm{d} a\right) \mathrm{d} t \mathrm{~d} x \\
& \leq\left\|\partial_{t} f\right\|_{L^{\infty}} \int|p(t, x)| c|v|\left(t, x-T_{\sigma, \delta}(y), a\right) \gamma(y) \mathrm{d} y \mathrm{~d} \sigma \mathrm{d} a \mathrm{~d} t \mathrm{~d} x \\
& \leq\left\|\partial_{t} f\right\|_{L^{\infty}}\|p\|_{L^{2}} \sqrt{\int\left(\int c|v|\left(t, x-T_{\sigma, \delta}(y), a\right) \gamma(y) \mathrm{d} y \mathrm{~d} \sigma \mathrm{d} a\right)^{2}} \mathrm{~d} t \mathrm{~d} x \\
& \leq C\left\|\partial_{t} f\right\|_{L^{\infty}}\|p\|_{L^{2}} \sqrt{\int c|v|^{2}\left(t, x-T_{\sigma, \delta}(y), a\right) \gamma(y) \mathrm{d} y \mathrm{~d} \sigma \mathrm{d} a \mathrm{~d} t \mathrm{~d} x} \\
& \leq C\left\|\partial_{t} f\right\|_{L^{\infty}}\|p\|_{L^{2}}\|v\|_{L^{2}\left(Q^{\prime}, \mathrm{d} c\right)}
\end{aligned}
$$

The second one is

$$
I_{2}=\int_{Q_{\tau}} p(t, x)\left(\int f\left(t+\eta, x-T_{\sigma, \delta}(y), a\right) \frac{\left[c v\left(t+\eta, x-T_{\sigma, \delta}(y), a\right)-c v\left(t, x-T_{\sigma, \delta}(y), a\right)\right]}{\eta} \gamma(y) \mathrm{d} y \mathrm{~d} \sigma \mathrm{d} a\right) \mathrm{d} t \mathrm{~d} x .
$$

To estimate this integral term, we split it into three parts introducing a mollification of $v_{\varepsilon}$ defined by

$$
\tilde{v}_{\varepsilon}(t, x, a)=\int_{D} v_{\varepsilon}(t, x+\chi(\varepsilon) z, a) \lambda(z) \mathrm{d} z
$$

where $\lambda$ is a mollifier on $R^{d}$.

Remark 11.5. We have to introduce $\tilde{v}_{\varepsilon}$ since $c$ and $v$ must be evaluated at the same time $t$.

Let us introduce

$$
I_{2}=I_{3}+I_{4}+I_{5}
$$

with

$$
\begin{gathered}
I_{3}=\int_{Q_{\tau}} p(t, x)\left(\int f_{\eta, y} \frac{\left[v\left(t+\eta, x-T_{\sigma, \delta}(y), a\right)-\tilde{v}_{\varepsilon}\left(t, x-T_{\sigma, \delta}(y), a\right)\right]}{\eta} c\left(t+\eta, x-T_{\sigma, \delta}(y), a\right) \gamma(y) \mathrm{d} y \mathrm{~d} \sigma \mathrm{d} a\right) \mathrm{d} t \mathrm{~d} x, \\
I_{4}=\int_{Q_{\tau}} p(t, x)\left(\int f_{\eta, y} \tilde{v}_{\varepsilon}\left(t, x-T_{\sigma, \delta}(y), a\right) \frac{\left[c\left(t+\eta, x-T_{\sigma, \delta}(y), a\right)-c\left(t, x-T_{\sigma, \delta}(y), a\right)\right]}{\eta} \gamma(y) \mathrm{d} y \mathrm{~d} \sigma \mathrm{d} a\right) \mathrm{d} t \mathrm{~d} x
\end{gathered}
$$
and

$$
I_{5}=\int_{Q_{\tau}} p(t, x)\left(\int f_{\eta, y} \frac{\left[\tilde{v}_{\varepsilon}\left(t, x-T_{\sigma, \delta}(y), a\right)-v\left(t, x-T_{\sigma, \delta}(y), a\right)\right]}{\eta} c\left(t, x-T_{\sigma, \delta}(y), a\right) \gamma(y) \mathrm{d} y \mathrm{~d} \sigma \mathrm{d} a\right) \mathrm{d} t \mathrm{~d} x
$$

where $f_{\eta, y}=f\left(t+\eta, x-T_{\sigma, \delta}(y), a\right)$. 
Let us first consider $I_{3}$.

$$
\begin{aligned}
& I_{3} \leq C\|f\|_{L^{\infty}\left(Q^{\prime}\right)} \mid\|p\|_{L^{2}} \\
& \times\left(\int_{Q_{\tau}}\left(\int\left(\frac{\left[v\left(t+\eta, x-T_{\sigma, \delta}(y), a\right)-\tilde{v}_{\varepsilon}\left(t, x-T_{\sigma, \delta}(y), a\right)\right]}{\eta}\right)^{2} c\left(t+\eta, x-T_{\sigma, \delta}(y), a\right) \gamma(y) \mathrm{d} y \mathrm{~d} \sigma \mathrm{d} a\right) \mathrm{d} t \mathrm{~d} x\right)^{\frac{1}{2}} .
\end{aligned}
$$

We have used Cauchy-Schwarz knowing that $f$ belongs to $L^{\infty}(Q)$ and $C(t, x)=1$. Moreover, thanks to Jensen's inequality, we have

$$
\begin{aligned}
\left(\int_{D} v_{\varepsilon}(t, x+\chi(\varepsilon) z, a) \lambda(z) \mathrm{d} z-v(t, x, a)\right)^{2} & =\left(\int_{D}\left(v_{\varepsilon}(t, x+\chi(\varepsilon) z, a)-v(t, x, a)\right) \lambda(z) \mathrm{d} z\right)^{2} \\
& \leq \int_{D}\left(v_{\varepsilon}(t, x+\chi(\varepsilon) z, a)-v(t, x, a)\right)^{2} \lambda(z) \mathrm{d} z
\end{aligned}
$$

Then, we obtain

$$
\begin{aligned}
& \int_{Q_{\tau}} \int\left|v\left(t+\eta, x-T_{\sigma, \delta}(y), a\right)-\tilde{v}_{\varepsilon}\left(t, x-T_{\sigma, \delta}(y), a\right)\right|^{2} c\left(t+\eta, x-T_{\sigma, \delta}(y), a\right) \mathrm{d} y \mathrm{~d} \sigma \mathrm{d} t \mathrm{~d} x \mathrm{~d} a \\
& \leq \int_{Q_{\tau}} \int\left|v\left(t+\eta, x-T_{\sigma, \delta}(y), a\right)-v_{\varepsilon}\left(t, x-T_{\sigma, \delta}(y)+\chi(\varepsilon) z, a\right)\right|^{2} \lambda(z) c\left(t+\eta, x-T_{\sigma, \delta}(y), a\right) \mathrm{d} z \mathrm{~d} y \mathrm{~d} \sigma \mathrm{d} t \mathrm{~d} x \mathrm{~d} a
\end{aligned}
$$

Thanks to inequalities (13) and (14), we get the following estimate

$$
I_{3} \leq\|p\|_{L^{2}(Q)} C \frac{\left(\varepsilon^{2}+\eta^{2}+\chi(\varepsilon)^{2}\right)^{\frac{1}{2}}}{\eta}
$$

We treat $I_{5}$ in a similar way and obtain

$$
I_{5} \leq\|p\|_{L^{2}(Q)} C \frac{\left(\varepsilon^{2}\right)^{\frac{1}{2}}}{\eta}
$$

To estimate $I_{4}$, we use the mean value theorem and the conservation of mass (6). Indeed,

$$
\begin{aligned}
& I_{4}=\int_{Q_{\tau}} p(t, x)\left(\iint_{0}^{1} f\left(t+\eta, x-T_{\sigma, \delta}(y), a\right) \tilde{v}_{\varepsilon}\left(t, x-T_{\sigma, \delta}(y), a\right) \partial_{t} c\left(t+\theta \eta, x-T_{\sigma, \delta}(y), a\right) \mathrm{d} \theta \mathrm{d} \gamma y \mathrm{~d} \sigma \mathrm{d} a\right) \mathrm{d} t \mathrm{~d} x \\
& =-\int_{Q_{\tau}} p(t, x)\left(\iint_{0}^{1} f\left(t+\eta, x-T_{\sigma, \delta}(y), a\right) \tilde{v}_{\varepsilon}\left(t, x-T_{\sigma, \delta}(y), a\right) \nabla_{x} \cdot(c v)\left(t+\theta \eta, x-T_{\sigma, \delta}(y), a\right) \mathrm{d} \theta \mathrm{d} \gamma y \mathrm{~d} \sigma \mathrm{d} a\right) \mathrm{d} t \mathrm{~d} x .
\end{aligned}
$$


An integration by parts with respect to $y$ enables us to transfer derivatives to $f$ and $\tilde{v}_{\varepsilon}$ (we recall that $T_{\sigma, \delta}(y)=$ $\delta y+\sigma \delta \omega$ and that $\left.\nabla_{x} \cdot(c v)=\frac{1}{\delta} \nabla_{y} \cdot(c v)\right)$. Here, all the estimates are made for $\delta$ fixed.

$$
\begin{aligned}
I_{4}= & \int_{Q_{\tau}} p(t, x)\left(\iint_{0}^{1} \frac{1}{\delta} \nabla_{y}(f)\left(t+\eta, x-T_{\sigma, \delta}(y), a\right) \tilde{v}_{\varepsilon}\left(t, x-T_{\sigma, \delta}(y), a\right) c v\left(t+\theta \eta, x-T_{\sigma, \delta}(y), a\right) \mathrm{d} \theta \mathrm{d} \gamma y \mathrm{~d} \sigma \mathrm{d} a\right) \mathrm{d} t \mathrm{~d} x \\
& +\int_{Q_{\tau}} p(t, x)\left(\iint_{0}^{1} \frac{1}{\delta}(f)\left(t+\eta, x-T_{\sigma, \delta}(y), a\right) \nabla_{y} \tilde{v}_{\varepsilon}\left(t, x-T_{\sigma, \delta}(y), a\right) c v\left(t+\theta \eta, x-T_{\sigma, \delta}(y), a\right) \mathrm{d} \theta \mathrm{d} \gamma y \mathrm{~d} \sigma \mathrm{d} a\right) \mathrm{d} t \mathrm{~d} x \\
= & I_{6}+I_{7} .
\end{aligned}
$$

The first term is bounded since

$$
I_{6} \leq \frac{1}{\delta}\|p\|_{L^{2}(Q)}\|\nabla f\|_{L^{\infty}(Q)}\|V\|_{L^{\infty}(Q)}\left(\int c\left|\tilde{v}_{\varepsilon}\right|^{2}\left(t+\eta \theta, x-T_{\sigma, \delta}(y), a\right) \mathrm{d}_{\gamma} y \mathrm{~d} a \mathrm{~d} \sigma \mathrm{d} \theta \mid \mathrm{d} t \mathrm{~d} x\right)^{1 / 2}
$$

and because the inequality (12) implies that $v_{\varepsilon}$ is uniformally bounded in $L^{2}\left(Q^{\prime}, \mathrm{d} c\right)$.

Let us study $I_{7}$. Let us notice that

$$
\begin{aligned}
\partial_{i} \tilde{v}_{\varepsilon}(t, x, a) & =\frac{1}{\chi(\varepsilon)} \int v_{\varepsilon}(t, x+\chi(\varepsilon) z, a) \partial_{i} \lambda(z) \mathrm{d} z \\
& =\frac{1}{\chi(\varepsilon)} \int\left[v_{\varepsilon}(t, x+\chi(\varepsilon) z, a)-v_{\varepsilon}(t, x, a)\right] \partial_{i} \lambda(z) \mathrm{d} z
\end{aligned}
$$

since $\int \partial_{i} \lambda(z) \mathrm{d} z=0$.

From (13), we have

$$
\begin{aligned}
& \frac{1}{\chi(\varepsilon)^{2}} \int_{Q_{\tau}} \int\left|v_{\varepsilon}\left(t, x-T_{\sigma, \delta}(y)+\chi(\varepsilon) z, a\right)-v_{\varepsilon}\left(t, x-T_{\sigma, \delta}(y), a\right)\right|^{2} c\left(t, x-T_{\sigma, \delta}(y), a\right) \mathrm{d} a \mathrm{~d}_{\gamma} y \mathrm{~d} \sigma \mathrm{d} t \mathrm{~d} x \\
& =\frac{1}{\chi(\varepsilon)^{2}} \int_{Q_{\tau}} \int\left|v_{\varepsilon}(t, x+\chi(\varepsilon) z, a)-v_{\varepsilon}(t, x, a)\right|^{2} c(t, x, a) \mathrm{d} a \mathrm{~d}_{\gamma} y \mathrm{~d} \sigma \mathrm{d} t \mathrm{~d} x \\
& \leq C \frac{1}{\chi(\varepsilon)^{2}}\left(\chi(\varepsilon)^{2}+\varepsilon^{2}\right)
\end{aligned}
$$

by translation invariance on the periodic cube. Since $\chi(\varepsilon) \geq \varepsilon$, we can write

$$
\int_{Q_{\tau}} \int\left|\nabla_{y} \tilde{v}_{\varepsilon}\left(t, x-T_{\sigma, \delta}(y), a\right)\right|^{2} c\left(t+\theta \eta, x-T_{\sigma, \delta}(y), a\right) \mathrm{d} \theta \mathrm{d}_{\gamma} y \mathrm{~d} \sigma \mathrm{d} a \mathrm{~d} t \mathrm{~d} x \leq C .
$$


And finally

$$
\begin{aligned}
I_{7} & \leq C \int_{Q_{\tau}}|p(t, x)| \frac{1}{\delta} V(t+\theta \eta)\left(\int\left|\nabla_{y} \tilde{v}_{\varepsilon}\left(t, x-T_{\sigma, \delta}(y), a\right)\right|^{2} c\left(t+\theta \eta, x-T_{\sigma, \delta}(y), a\right) \mathrm{d} \theta \mathrm{d}_{\gamma} y \mathrm{~d} \sigma \mathrm{d} a\right)^{\frac{1}{2}} \mathrm{~d} t \mathrm{~d} x \\
& \leq C\|p\|_{L^{2}(Q)}\left(\int_{Q_{\tau}}\left(\int\left|\nabla_{x} \tilde{v}_{\varepsilon}\left(t, x-T_{\sigma, \delta}(y), a\right)\right|^{2} c\left(t+\theta \eta, x-T_{\sigma, \delta}(y), a\right) \mathrm{d} \theta \mathrm{d}_{\gamma} y \mathrm{~d} \sigma \mathrm{d} a\right) \mathrm{d} t \mathrm{~d} x\right)^{\frac{1}{2}} \\
& \leq C\|p\|_{L^{2}(Q)},
\end{aligned}
$$

since $V$ belongs to $L^{\infty}$.

Finally,

$$
\left\langle p(t, x) ; \partial_{t} g(t, x)\right\rangle=\lim _{\eta} A_{\eta}
$$

where

$$
\begin{aligned}
A_{\eta} & =\int_{Q} p(t, x) \frac{[g(t+\eta, x)-g(t, x)]}{\eta} \mathrm{d} t \mathrm{~d} x \\
& \leq C\left(1+\frac{\left(\varepsilon^{2}+\chi(\varepsilon)^{2}\right)^{\frac{1}{2}}}{\eta}\right)\|p\|_{L^{2}(Q)}
\end{aligned}
$$

for any $\varepsilon$. Passing to the limit when $\varepsilon \rightarrow 0$, we obtain

$$
A_{\eta} \leq C\|p\|_{L^{2}(Q)}
$$

for every $\eta$. So when $\eta \rightarrow 0$

$$
\left\langle p(t, x) ; \partial_{t} g(t, x)\right\rangle \leq C\|p\|_{L^{2}(Q)} .
$$

\subsection{Terms involving $\partial_{t} H_{\varepsilon}$}

It remains to pass to the limit in the term involving a time derivative

$$
\int_{Q^{\prime}}-\partial_{t} H_{\varepsilon}(t, x+\delta y+\sigma \delta \omega) \cdot \omega f(t, x, a) \gamma(y) \mathrm{d} c \mathrm{~d} \sigma \mathrm{d} y=\int_{Q}-\partial_{t} H_{\varepsilon}(t, x) h(t, x) \cdot \omega \mathrm{d} t \mathrm{~d} x,
$$

where

$$
h(t, x)=\int f(t, x+\delta y+\sigma \delta \omega, a) \gamma(y) c(t, x+\delta y+\sigma \delta \omega, \mathrm{d} a) \mathrm{d} y \mathrm{~d} \sigma .
$$

The function $h$ is smooth with respect to $x$ thanks to the mollification. Moreover, we use (7) and we obtain

$$
\begin{aligned}
\partial_{t} h(t, x)= & \int\left(\partial_{t}+v(t, x+\delta y+\sigma \delta \omega, a) \cdot \nabla_{x}\right) f(t, x+\delta y+\sigma \delta \omega, a) c(t, x+\delta y+\sigma \delta \omega, \mathrm{d} a) \gamma(y) \mathrm{d} y \mathrm{~d} \sigma \\
& -\nabla_{x} \cdot \int v(t, x+\delta y+\sigma \delta \omega, a) f(t, x+\delta y+\sigma \delta \omega, a) c(t, x+\delta y+\sigma \delta \omega, \mathrm{d} a) \gamma(y) \mathrm{d} y \mathrm{~d} \sigma
\end{aligned}
$$

which belongs to $L^{2}(Q)$. Then $h$ belongs to $G$ and we can pass to the limit when $\varepsilon \rightarrow 0$. 


\section{Partial Regularity of the Field $H$}

In this section, it is shown that the limit field $H$ has some partial regularity, namely $E=\partial_{t} H$ is a locally bounded measure and $B=-\operatorname{curl} H$ is square integrable with respect to the Lebesgue measure.

\section{1. $\boldsymbol{B}=-\operatorname{curl} \boldsymbol{H}$ is Lebesgue square integrable}

We show here that $B=-\operatorname{curl} H$ belongs to $L^{2}(Q)$ thanks to the Riesz theorem. So we have to prove the inequality

$$
\langle\operatorname{curl} H(t, x) ; f(t, x)\rangle \leq C\|f\|_{L^{2}(Q)} .
$$

For that purpose, we use a formulation of the curl involving finite differences and which vanish for gradients. We then introduce

$$
\begin{aligned}
\left(\operatorname{curl}_{\delta} f\right)_{1}= & \int_{0}^{1} \frac{\left[f_{3}(t, x, y+\delta, z+\sigma \delta)-f_{3}(t, x, y, z+\sigma \delta)\right]}{\delta} \mathrm{d} \sigma \\
& -\int_{0}^{1} \frac{\left[f_{2}(t, x, y+\tau \delta, z+\delta)-f_{2}(t, x, y+\tau \delta, z)\right]}{\delta} \mathrm{d} \tau
\end{aligned}
$$

We have $(\operatorname{curl} f)_{1}=\lim _{\delta \rightarrow 0}\left(\operatorname{curl}_{\delta} f\right)_{1}$ and $\operatorname{curl}_{\delta} f$ vanishes as $f$ is a gradient.

Indeed, using the mean value theorem, we have

$$
\left(\operatorname{curl}_{\delta} f\right)_{1}=\int_{0}^{1} \int_{0}^{1} \partial_{2} f_{3}(t, x, y+\tau \delta, z+\sigma \delta)-\partial_{3} f_{2}(t, x, y+\tau \delta, z+\sigma \delta) \mathrm{d} \sigma \mathrm{d} \tau
$$

which ensures that $\operatorname{curl}_{\delta} f$ vanishes as $f$ is a gradient and that

$$
\begin{aligned}
(\operatorname{curl} f)_{1}= & \lim _{\delta \rightarrow 0} \int_{0}^{1} \frac{\left[f_{3}(t, x, y+\delta, z+\sigma \delta)-f_{3}(t, x, y, z+\sigma \delta)\right]}{\delta} \mathrm{d} \sigma \\
& -\int_{0}^{1} \frac{\left[f_{2}(t, x, y+\tau \delta, z+\delta)-f_{2}(t, x, y+\tau \delta, z)\right]}{\delta} \mathrm{d} \tau
\end{aligned}
$$

as $f$ is smooth. To obtain an estimate involving only $\|f\|_{L^{2}}$, we study

$$
\begin{aligned}
\int_{Q}\left(\operatorname{curl}_{\delta} H\right)_{1} f_{1}= & \int_{Q}\left[\int_{0}^{1} \frac{\left[H_{3}(t, x, y+\delta, z+\sigma \delta)-H_{3}(t, x, y, z+\sigma \delta)\right]}{\delta} \mathrm{d} \sigma\right. \\
& \left.-\int_{0}^{1} \frac{\left[H_{2}(t, x, y+\tau \delta, z+\delta)-H_{2}(t, x, y+\tau \delta, z)\right]}{\delta} \mathrm{d} \tau\right] f_{1}(t, x) \mathrm{d} t \mathrm{~d} x .
\end{aligned}
$$


Since $H=\lim _{\varepsilon \rightarrow 0} H_{\varepsilon}$, we have

$$
\begin{aligned}
\int_{Q}\left(\operatorname{curl}_{\delta} H\right)_{1} f_{1}= & \lim _{\varepsilon \rightarrow 0} \int_{Q}\left[\int_{0}^{1} \frac{\left[H_{3}^{\varepsilon}(t, x, y+\delta, z+\sigma \delta)-H_{3}^{\varepsilon}(t, x, y, z+\sigma \delta)\right]}{\delta} \mathrm{d} \sigma\right. \\
& \left.-\int_{0}^{1} \frac{\left[H_{2}^{\varepsilon}(t, x, y+\tau \delta, z+\delta)-H_{2}^{\varepsilon}(t, x, y+\tau \delta, z)\right]}{\delta} \mathrm{d} \tau\right] f_{1}(t, x) \mathrm{d} c \\
= & \lim _{\varepsilon \rightarrow 0} \int_{Q_{\tau}^{\prime}}\left[\int_{0}^{1} \frac{\left[v_{3}^{\varepsilon}(t, x, y+\delta, z+\sigma \delta, a)-v_{3}^{\varepsilon}(t, x, y, z+\sigma \delta, a)\right]}{\delta} \mathrm{d} \sigma\right. \\
& \left.-\int_{0}^{1} \frac{\left[v_{2}^{\varepsilon}(t, x, y+\tau \delta, z+\delta, a)-v_{2}^{\varepsilon}(t, x, y+\tau \delta, z, a)\right]}{\delta} \mathrm{d} \tau\right] f_{1}(t, x) \mathrm{d} c
\end{aligned}
$$

since $\operatorname{curl}_{\delta}\left(\nabla \phi_{\varepsilon}\right)=0$ and $f$ is zero outside $Q_{\tau}$.

Moreover, equation (13) implies that

$$
\int_{Q_{\tau}^{\prime}} \int_{0}^{1}\left(\frac{\left[v_{3}^{\varepsilon}(t, x, y+\delta, z+\sigma \delta, a)-v_{3}^{\varepsilon}(t, x, y, z+\sigma \delta, a)\right]^{2}}{\delta^{2}}\right) \mathrm{d} \sigma \mathrm{d} c \leq C\left(\frac{\varepsilon^{2}+\delta \chi(\varepsilon)}{\delta^{2}}+1\right) .
$$

Then, for every $\varepsilon$ and every $\delta$

$$
\int_{Q} \operatorname{curl}_{\delta} H_{\varepsilon} \cdot f \leq C\left(\frac{\varepsilon^{2}+\delta \chi(\varepsilon)}{\delta^{2}}+1\right)^{\frac{1}{2}}\|f\|_{L^{2}(Q)} .
$$

So passing to the limit when $\varepsilon \rightarrow 0$ for a fixed $\delta$, we obtain

$$
\int_{Q} \operatorname{curl}_{\delta} H \cdot f \leq C\|f\|_{L^{2}(Q)}
$$

Remark 12.1. let us notice that

$$
\int_{Q} \operatorname{curl}_{\delta} H \cdot f=\int_{Q} H \cdot \operatorname{curl}_{\delta} f .
$$

Since $C$ does not depend on $\delta$, the estimate remains true at the limit and then

$$
\langle H(t, x) ; \operatorname{curl} f(t, x)\rangle=\langle\operatorname{curl} H(t, x) ; f(t, x)\rangle \leq C\|f\|_{L^{2}(Q)} .
$$

\section{2. $E=\partial_{t} H$ is a locally bounded measure}

Let us now show that $E$ is a measure locally bounded on $Q$. Let $\zeta(t)$ be a smooth function, we study

$$
\int_{Q} \zeta(t) \frac{\left|H_{\varepsilon}(t+\eta, x)-H_{\epsilon}(t, x)\right|}{\eta} \mathrm{d} t \mathrm{~d} x
$$


or more precisely

$$
I=\int_{Q} \zeta(t) \frac{\left|\int_{0}^{1}\left(H_{\varepsilon}(t+\eta, x+\sigma \delta e)-H_{\epsilon}(t, x+\sigma \delta e)\right) \cdot e \mathrm{~d} \sigma\right|}{\eta} \mathrm{d} t \mathrm{~d} x
$$

where $\eta$ is small enough so that $\zeta(t-\theta \eta)$ is compactly supported in time and let $e$ be a fixed unit vector in $R^{d}$.

Let $\tau$ be small enough so that $\zeta$ vanishes outside $Q_{\tau}^{\prime}$, we have

$$
\begin{aligned}
I= & \int_{Q_{\tau}^{\prime}} \zeta(t) \frac{\left|\int_{0}^{1}\left(H_{\varepsilon}(t+\eta, x+\sigma \delta e)-H_{\epsilon}(t, x+\sigma \delta e)\right) \cdot e \mathrm{~d} \sigma\right|}{\eta} \mathrm{d} c \\
\leq & \int_{Q_{\tau}^{\prime}} \zeta(t) \frac{\left|\int_{0}^{1}\left(\left(H_{\varepsilon}+\nabla \phi_{\varepsilon}\right)(t+\eta, x+\sigma \delta e, a)-\left(H_{\epsilon}+\nabla \phi_{\varepsilon}\right)(t, x+\sigma \delta e, a)\right) \cdot e \mathrm{~d} \sigma\right|}{\eta} \mathrm{d} c \\
& +\int_{Q_{\tau}^{\prime}} \zeta(t) \frac{\left|\int_{0}^{1}\left(\nabla \phi_{\varepsilon}(t+\eta, x+\sigma \delta e, a)-\nabla \phi_{\varepsilon}(t, x+\sigma \delta e, a)\right) \cdot e \mathrm{~d} \sigma\right|}{\eta} \mathrm{d} c \\
= & I_{1}+I_{2} .
\end{aligned}
$$

Thanks to $(13)$

$$
I_{1} \leq C \frac{\left(\varepsilon^{2}+\delta^{2}+\delta \chi(\varepsilon)+\eta^{2}+\eta \chi^{\prime}(\varepsilon)\right)^{\frac{1}{2}}}{\eta}
$$

On the other hand, thanks to the mean value theorem, we have

$$
I_{2}=\int_{Q_{\tau}^{\prime}} \zeta(t) \frac{\left|\int_{0}^{1}\left(\partial_{t} \phi_{\varepsilon}(t+\theta \eta, x+\delta e, a)-\partial_{t} \phi_{\varepsilon}(t+\theta \eta, x, a)\right) \mathrm{d} \theta\right|}{\delta} \mathrm{d} c
$$

and then

$$
\begin{aligned}
I_{2} \leq & \int_{Q_{\tau}^{\prime}} \zeta(t) \frac{\left|\int_{0}^{1}\left(\lambda^{\varepsilon}(t+\theta \eta, x+\delta e, a)-\lambda^{\varepsilon}(t+\theta \eta, x, a)\right) \mathrm{d} \theta\right|}{\delta} \mathrm{d} c \\
& +\int_{Q_{\tau}^{\prime}} \zeta(t) \frac{\left|\int_{0}^{1}\left(\frac{1}{2}\left|\nabla \phi_{\varepsilon}+H_{\varepsilon}\right|^{2}(t+\theta \eta, x+\delta e, a)-\frac{1}{2}\left|\nabla \phi_{\varepsilon}+H_{\varepsilon}\right|^{2}(t+\theta \eta, x, a)\right) \mathrm{d} \theta\right|}{\delta} \mathrm{d} c \\
= & I_{3}+I_{4}
\end{aligned}
$$

where $\lambda^{\varepsilon}(t, x, a)=-\partial_{t} \phi_{\varepsilon}(t, x, a)-\frac{1}{2}\left|\nabla \phi_{\varepsilon}+H_{\varepsilon}\right|^{2}(t, x, a)$.

We estimate $I_{4}$ as $I_{1}$ writing

$$
I_{4} \leq C \frac{\left(\varepsilon^{2}+\delta^{2}+\delta \chi(\varepsilon)+\eta^{2}+\eta \chi^{\prime}(\varepsilon)\right)^{\frac{1}{2}}}{\delta}
$$


Since $\lambda^{\varepsilon} \geq 0$ and $\int \lambda^{\varepsilon} \mathrm{d} c \leq \varepsilon^{2}$, we have

$$
\begin{aligned}
I_{3} & \leq \int_{Q_{\tau}^{\prime}} \int_{0}^{1} \zeta(t) \frac{\left(\lambda^{\varepsilon}(t+\theta \eta, x+\delta e, a)+\lambda^{\varepsilon}(t+\theta \eta, x, a)\right)}{\delta} \mathrm{d} \theta \mathrm{d} c \\
& \leq 2 \frac{\varepsilon^{2}}{\delta}+\int_{Q_{\tau}^{\prime}} \int_{0}^{1} \zeta(t) \frac{\left(\lambda^{\varepsilon}(t+\theta \eta, x+\delta e, a)+\lambda^{\varepsilon}(t+\theta \eta, x, a)-2 \lambda^{\varepsilon}(t, x, a)\right)}{\delta} \mathrm{d} \theta \mathrm{d} c \\
& =2 \frac{\varepsilon^{2}}{\delta}-I_{5}-I_{6}
\end{aligned}
$$

where

$$
I_{5}=\int \zeta(t) \frac{\left(\partial_{t} \phi_{\varepsilon}(t+\theta \eta, x+\delta e, a)+\partial_{t} \phi_{\varepsilon}(t+\theta \eta, x, a)-2 \partial_{t} \phi_{\varepsilon}(t, x, a)\right)}{\delta} \mathrm{d} \theta \mathrm{d} c
$$

and

$$
I_{6}=\frac{1}{2 \delta} \int \zeta(t)\left(\left|\nabla \phi_{\varepsilon}+H_{\varepsilon}\right|^{2}(t+\theta \eta, x+\delta e, a)+\left|\nabla \phi_{\varepsilon}+H_{\varepsilon}\right|^{2}(t+\theta \eta, x, a)-2\left|\nabla \phi_{\varepsilon}+H_{\varepsilon}\right|^{2}(t, x, a)\right) \mathrm{d} \theta \mathrm{d} c .
$$

The term $I_{6}$ can be estimated as $I_{4}$ and

$$
I_{5}=I_{7}+I_{8}
$$

where

$$
I_{7}=\frac{1}{\delta} \int\left[\zeta(t)\left(\partial_{t} \phi_{\varepsilon}(t+\theta \eta, x+\delta e, a)+\partial_{t} \phi_{\varepsilon}(t+\theta \eta, x, a)\right)-2 \zeta(t-\eta \theta) \partial_{t} \phi_{\varepsilon}(t, x, a)\right] \mathrm{d} \theta \mathrm{d} c
$$

and

$$
\begin{aligned}
I_{8}= & \frac{1}{\delta} \int 2(\zeta(t-\eta \theta)-\zeta(t)) \partial_{t} \phi_{\varepsilon}(t, x, a) \mathrm{d} \theta \mathrm{d} c \\
= & \frac{1}{\delta} \int 2(\zeta(t-\eta \theta)-\zeta(t))\left(-\lambda^{\varepsilon}(t, x, a)\right) \mathrm{d} \theta \mathrm{d} c \\
& \left.-\frac{1}{\delta} \int(\zeta(t-\eta \theta)-\zeta(t))\left|\nabla \phi_{\varepsilon}+H_{\varepsilon}\right|^{2}(t, x, a)\right) \mathrm{d} \theta \mathrm{d} c \\
\leq & C \frac{\eta}{\delta} .
\end{aligned}
$$

Indeed,

$$
\int(\zeta(t-\eta \theta)-\zeta(t))\left(\lambda^{\varepsilon}(t, x, a)\right) \mathrm{d} \theta \mathrm{d} c \leq \int \operatorname{Lip}(\zeta) \eta \theta\left(\lambda^{\varepsilon}(t, x, a)\right) \mathrm{d} \theta \mathrm{d} c \leq C \eta \varepsilon^{2}
$$

and

$$
\left.\left.\int(\zeta(t-\eta \theta)-\zeta(t))\left|\nabla \phi_{\varepsilon}+H_{\varepsilon}\right|^{2}(t, x, a)\right) \mathrm{d} \theta \mathrm{d} c \leq \int \operatorname{Lip}(\zeta) \eta \theta\left|\nabla \phi_{\varepsilon}+H_{\varepsilon}\right|^{2}(t, x, a)\right) \mathrm{d} \theta \mathrm{d} c \leq C \eta .
$$

Then

$$
\begin{aligned}
I_{7} & =\frac{1}{\delta} \int\left[\zeta(t)\left(\partial_{t} \phi_{\varepsilon}(t+\theta \eta, x+\delta e, a)+\partial_{t} \phi_{\varepsilon}(t+\theta \eta, x, a)\right)-2 \zeta(t-\eta \theta) \partial_{t} \phi_{\varepsilon}(t, x, a)\right] \mathrm{d} \theta \mathrm{d} c \\
& =I_{9}+2 I_{10}
\end{aligned}
$$


where

$$
\begin{aligned}
I_{9}= & \frac{1}{\delta} \int \zeta(t)\left[\partial_{t} \phi_{\varepsilon}(t+\theta \eta, x+\delta e, a)-\partial_{t} \phi_{\varepsilon}(t+\theta \eta, x, a)\right] \mathrm{d} \theta \mathrm{d} c \\
= & \int \zeta(t) \int_{0}^{1} \partial_{t} \nabla \phi_{\varepsilon}(t+\theta \eta, x+\sigma \delta e, a) \cdot e \mathrm{~d} \theta \mathrm{d} \sigma \mathrm{d} c \\
= & \int \zeta(t) \int_{0}^{1} \frac{\left(\left(\nabla \phi_{\varepsilon}+H_{\varepsilon}\right)(t+\eta, x+\sigma \delta e, a)-\left(\nabla \phi_{\varepsilon}+H_{\varepsilon}\right)(t, x+\sigma \delta e, a)\right)}{\eta} \cdot e \mathrm{~d} \sigma \mathrm{d} c \\
& -\int \zeta(t) \int_{0}^{1} \partial_{t} H_{\varepsilon}(t+\theta \eta, x+\sigma \delta e) \cdot e \mathrm{~d} \theta \mathrm{d} \sigma \mathrm{d} t \mathrm{~d} x \\
\leq & C \frac{\left(\varepsilon^{2}+\delta^{2}+\delta \chi(\varepsilon)+\eta^{2}+\eta \chi^{\prime}(\varepsilon)\right)^{\frac{1}{2}}}{\eta}+C
\end{aligned}
$$

(since $\partial_{t} H_{\varepsilon}$ is a bounded sequence in $G^{\prime}$, we have $\int \zeta(t) \partial_{t} H_{\varepsilon}(t, x) \mathrm{d} t \mathrm{~d} x \leq C$ ).

$$
I_{10}=\frac{1}{\delta} \int\left[\zeta(t) \partial_{t} \phi_{\varepsilon}(t+\theta \eta, x, a)-\zeta(t-\eta \theta) \partial_{t} \phi_{\varepsilon}(t, x, a)\right] \mathrm{d} \theta \mathrm{d} c
$$

Let $Z(t)=\zeta(t-\eta \theta) \partial_{t} \phi_{\varepsilon}(t, x, a)$, we have

$$
I_{10}=\frac{1}{\delta} \int(Z(t+\eta \theta)-Z(t)) \mathrm{d} \theta \mathrm{d} c=\frac{\eta}{\delta} \iint_{0}^{1} \partial_{t} Z(t+\sigma \eta \theta) \theta \mathrm{d} \sigma \mathrm{d} \theta \mathrm{d} c .
$$

And then

$$
I_{10}=\frac{\eta}{\delta} \iint_{0}^{1} \partial_{t}\left[\zeta(t-(1-\sigma) \eta \theta) \partial_{t} \phi_{\varepsilon}(t+\sigma \theta \eta, x, a)\right] \theta \mathrm{d} \sigma \mathrm{d} \theta \mathrm{d} c
$$

or using (6)

$$
\begin{aligned}
I_{10}= & -\frac{\eta}{\delta} \iint_{0}^{1} \zeta(t-(1-\sigma) \eta \theta) \partial_{t} \nabla \phi_{\varepsilon}(t+\sigma \theta \eta, x, a) \cdot v(t, x, a) \theta \mathrm{d} \sigma \mathrm{d} \theta \mathrm{d} c \\
= & -\frac{\eta}{\delta} \iint_{0}^{1} \zeta(t-(1-\sigma) \eta \theta) \partial_{t}\left(\nabla \phi_{\varepsilon}+H_{\varepsilon}\right)(t+\sigma \theta \eta, x, a) \cdot v(t, x, a) \theta \mathrm{d} \sigma \mathrm{d} \theta \mathrm{d} c \\
& +\frac{\eta}{\delta} \iint_{0}^{1} \zeta(t-(1-\sigma) \eta \theta) \partial_{t} H_{\varepsilon}(t+\sigma \theta \eta, x) \cdot u(t, x) \theta \mathrm{d} \sigma \mathrm{d} \theta \mathrm{d} t \mathrm{~d} x \\
\leq & I_{11}+I_{12}+C \frac{\eta}{\delta}
\end{aligned}
$$

where

$$
\begin{aligned}
I_{11} & =-\frac{\eta}{\delta} \iint_{0}^{1} \partial_{t}\left[\zeta(t-(1-\sigma) \eta \theta)\left(\nabla \phi_{\varepsilon}+H_{\varepsilon}\right)(t+\sigma \theta \eta, x, a)\right] \cdot v(t, x, a) \theta \mathrm{d} \theta \mathrm{d} \sigma \mathrm{d} c \\
& =-\frac{\eta}{\delta} \int \frac{\left[\zeta(t)\left(\nabla \phi_{\varepsilon}+H_{\varepsilon}\right)(t+\theta \eta, x, a)-\zeta(t-\eta \theta)\left(\nabla \phi_{\varepsilon}+H_{\varepsilon}\right)(t, x, a)\right]}{\eta} \cdot v(t, x, a) \mathrm{d} \theta \mathrm{d} c .
\end{aligned}
$$


Then we have

$$
I_{11} \leq C\left(\frac{\eta}{\delta} \frac{\left(\varepsilon^{2}+\delta^{2}+\delta \chi(\varepsilon)+\eta^{2}+\eta \chi^{\prime}(\varepsilon)\right)^{\frac{1}{2}}}{\eta}\right)
$$

and

$$
\begin{aligned}
I_{12} & =\frac{\eta}{\delta} \iint_{0}^{1} \zeta^{\prime}(t-(1-\sigma) \eta \theta)\left(\nabla \phi_{\varepsilon}+H_{\varepsilon}\right)(t+\sigma \theta \eta, x, a) \cdot v(t, x, a) \theta \mathrm{d} \theta \mathrm{d} \sigma \mathrm{d} c \\
& \leq C \frac{\eta}{\delta} .
\end{aligned}
$$

Finally, we have shown that

$$
\begin{aligned}
\int_{Q_{\tau}}\left|\int_{0}^{1} \frac{\left(H_{\varepsilon}(t+\eta, x+\sigma \delta e)-H_{\varepsilon}(t, x+\sigma \delta e)\right)}{\eta} \mathrm{d} \sigma\right| \mathrm{d} x \mathrm{~d} t \\
\leq C\left(1+\frac{\eta}{\delta}+\frac{\left(\varepsilon^{2}+\delta^{2}+\delta \chi(\varepsilon)+\eta^{2}+\eta \chi^{\prime}(\varepsilon)\right)^{\frac{1}{2}}}{\eta}+\frac{\left(\varepsilon^{2}+\delta^{2}+\delta \chi(\varepsilon)+\eta^{2}+\eta \chi^{\prime}(\varepsilon)\right)^{\frac{1}{2}}}{\delta}\right) .
\end{aligned}
$$

When $\varepsilon \rightarrow 0$ with $\eta=\delta$, we obtain

$$
\frac{1}{\eta} \int\langle H(t, x) ; f(t-\eta, x-\sigma \delta e)-f(t, x-\sigma \delta e)\rangle \mathrm{d} \sigma \leq C \sup |f|
$$

for every test function $f$ and then, $\partial_{t} H$ is a locally bounded measure (we choose $\zeta$ so that $\zeta=1$ on the support of $f$ ).

\section{Final Step: Derivation of the optimality equations}

We have justified the limit $\varepsilon \rightarrow 0$ and we have obtained

$$
\int\left(\partial_{t} f(t, x, a)+v(t, x, a) \cdot \nabla_{x} f(t, x, a)\right) v(t, x, a) \cdot \omega \mathrm{d} c-\int \partial_{t} H \cdot \omega h(t, x)+\int \operatorname{curl} H(t, x) \wedge g(t, x) \cdot \omega \leq C_{f} \delta .
$$

We recall that

$$
h(t, x)=h_{\delta}(t, x)=\iint_{-1}^{1} f(t, x-\delta y-\delta \sigma \omega, a) c(t, x-\delta y-\delta \sigma \omega, a) \gamma(y) \mathrm{d} y \mathrm{~d} \sigma \mathrm{d} a
$$

and

$$
g(t, x)=g_{\delta}(t, x)=\int f(t, x-\delta y-\delta \sigma \omega, a) v(t, x-\delta y-\delta \sigma \omega, a) c(t, x-\delta y-\delta \sigma \omega, a) \gamma(y) \mathrm{d} y \mathrm{~d} \sigma \mathrm{d} a .
$$

It remains to identify the limit $\delta \rightarrow 0$. For that purpose, we define

$$
\begin{aligned}
c_{\omega, \delta, \gamma} & =\int_{-1}^{1} \int c(t, x-\sigma \delta \omega-\delta y, a) \gamma(y) \mathrm{d} y \mathrm{~d} \sigma \\
m_{\omega, \delta, \gamma} & =\int_{-1}^{1} \int v c(t, x-\sigma \delta \omega-\delta y, a) \gamma(y) \mathrm{d} y \mathrm{~d} \sigma .
\end{aligned}
$$


We know that $m_{\omega, \delta, \gamma}$ converges up to a subsequence in $w-L^{2}\left(Q^{\prime}\right)$. Moreover, for every smooth $f$,

$$
\int f(t, x, a) m_{\omega, \delta, \gamma} \rightarrow \int f(t, x, a) v(t, x, a) \mathrm{d} c
$$

Indeed

$$
\left|\int f(t, x, a)\left(\mathrm{d} m_{\omega, \delta, \gamma}-\mathrm{d} m\right)\right|=\left|\int(f(t, x-\delta y-\sigma \delta \omega, a)-f(t, x, a)) \mathrm{d} m\right| \leq C \delta
$$

Moreover curl $H \in L^{2}$. Thus there is a sequence of smooth field $K_{\varepsilon}(t, x)$ approximating $\operatorname{curl} H$ in $L^{2}$ norm. Using this sequence, we show

$$
\int \operatorname{curl} H(t, x) f(t, x-\delta y-\sigma \delta \omega, a) v(t, x-\delta y-\sigma \delta \omega, a) c(t, x-\delta y-\sigma \delta \omega, \mathrm{d} a) \mathrm{d} t \mathrm{~d} x \rightarrow \int \operatorname{curl} H(t, x) f(t, x, a) \mathrm{d} m
$$

since denoting

$$
\begin{aligned}
I= & \mid \int \operatorname{curl} H(t, x) f(t, x-\delta y-\sigma \delta \omega, a) v(t, x-\delta y-\sigma \delta \omega, a) c(t, x-\delta y-\sigma \delta \omega, \mathrm{d} a) \mathrm{d} t \mathrm{~d} x \\
& -\int \operatorname{curl} H(t, x) f \mathrm{~d} m \mid
\end{aligned}
$$

we have

$$
I \leq I_{1}+I_{2}+I_{3}+I_{4}
$$

where

$$
\begin{gathered}
I_{1}=\left|\int \operatorname{curl} H(t, x)(f(t, x-\delta y-\sigma \delta \omega, a)-f(t, x, a)) v(t, x-\delta y-\sigma \delta \omega, a) c(t, x-\delta y-\sigma \delta \omega, \mathrm{d} a) \mathrm{d} t \mathrm{~d} x\right| \\
\leq \delta\|\operatorname{curl} H\|_{L^{2}}\|\nabla f\|_{L^{\infty}}\left(\int v^{2}(t, x-\delta y-\sigma \delta \omega, a) c(t, x-\delta y-\sigma \delta \omega, \mathrm{d} a) \mathrm{d} t \mathrm{~d} x\right)^{\frac{1}{2}}, \\
I_{2}=\left|\int\left(\operatorname{curl} H(t, x)-K^{\varepsilon}(t, x)\right) f(t, x, a) v(t, x-\delta y-\sigma \delta \omega, a) c(t, x-\delta y-\sigma \delta \omega, \mathrm{d} a) \mathrm{d} t \mathrm{~d} x\right| \\
\leq\left\|\operatorname{curl} H-K^{\varepsilon}\right\|_{L^{2}(Q)}\|f\|_{L^{\infty}}\left(\int v^{2}(t, x-\delta y-\sigma \delta \omega, a) c(t, x-\delta y-\sigma \delta \omega, \mathrm{d} a) \mathrm{d} t \mathrm{~d} x\right)^{\frac{1}{2}} \\
\leq C\left\|\operatorname{curl} H-K^{\varepsilon}\right\|_{L^{2}(Q)}\|f\|_{L^{\infty}}, \\
I_{3}=\left|\int K^{\varepsilon}(t, x) f(t, x, a)(v(t, x-\delta y-\sigma \delta \omega, a) c(t, x-\delta y-\sigma \delta \omega, \mathrm{d} a)-v(t, x, a) c(t, x, \mathrm{~d} a)) \mathrm{d} t \mathrm{~d} x\right| \\
\leq C \delta\left\|\nabla\left(K^{\varepsilon} f\right)\right\|_{L^{\infty}} \leq C_{\varepsilon} \delta
\end{gathered}
$$


and

$$
\begin{aligned}
I_{4} & =\left|\int\left(\operatorname{curl} H(t, x)-K^{\varepsilon}(t, x)\right) f(t, x, a) v(t, x, a) \mathrm{d} c\right| \\
& \leq\left\|\operatorname{curl} H-K^{\varepsilon}\right\|_{L^{2}(Q)}|| f \|_{L^{\infty}}\left(\int|v|^{2} \mathrm{~d} c\right)^{\frac{1}{2}}
\end{aligned}
$$

Finally,

$$
\begin{aligned}
I & \leq C_{\varepsilon} \delta+C\left\|\operatorname{curl} H-K^{\varepsilon}\right\|_{L^{2}(Q)}|| f \|_{L^{\infty}} \\
& \leq C \varepsilon+C_{\varepsilon} \delta
\end{aligned}
$$

for every $\varepsilon$. So when $\delta \rightarrow 0$ and then $\varepsilon \rightarrow 0$, we obtain the result.

But it is impossible to define the product $c \partial_{t} H$. We have to do the difference between $c$ and the weak-* limit of $c_{\omega, \delta, \gamma}$ in $L^{\infty}\left(\left|\partial_{t} H\right| ; C(A)^{\prime}\right)$ when $\delta \rightarrow 0$. We have

$$
\left|h(t, x)-\int f(t, x, a) c_{\omega, \delta, \gamma}(t, x, \mathrm{~d} a)\right| \leq C_{f} \delta
$$

and since $\partial_{t} H$ is locally bounded, we obtain

$$
\left|\int \partial_{t} H(t, x)\left(h(t, x)-\int f(t, x, a) c_{\omega, \delta, \gamma}(t, x, \mathrm{~d} a)\right) \mathrm{d} t \mathrm{~d} x\right| \leq C \delta .
$$

Let $c_{\omega, \gamma}$ be the limit of $c_{\omega, \delta, \gamma}$ in $w * L^{\infty}\left(\left|\partial_{t} H\right| ; C(A)^{\prime}\right)$, we have (1.1) which implies that

$$
\int \partial_{t} H(t, x) \int f(t, x, a) c_{\omega, \delta, \gamma}(t, x, \mathrm{~d} a) \mathrm{d} t \mathrm{~d} x \rightarrow \int \partial_{t} H \int f(t, x, a) c_{\omega, \gamma}(t, x, \mathrm{~d} a) \mathrm{d} t \mathrm{~d} x .
$$

Finally, we obtained

$$
\int \partial_{t} H \cdot \omega \int f(t, x, a) c_{\omega, \gamma}(t, x, \mathrm{~d} a) \mathrm{d} t \mathrm{~d} x=-\int v \cdot \omega\left(\partial_{t} f+v \cdot \nabla_{x} f\right) \mathrm{d} c+v \wedge \operatorname{curl} H f \cdot \omega \mathrm{d} c .
$$

Since the right-hand term does not depend on $\gamma$ and depend linearly on $\omega, c_{\omega, \gamma}$ can not depend on $\gamma$ or $\omega$. Let us denote $\underline{c}=c_{\omega, \gamma}$, we have, up to a subsequence

$$
\partial_{t} v+c v \cdot \nabla_{x} v-\underline{c} \partial_{t} H+c v \wedge \operatorname{curl} H=0 .
$$

We can show the convergence of the whole sequence since denoting

$$
F_{\varepsilon, \delta}=-\int \partial_{t} H_{\varepsilon} \cdot \omega h+\int \operatorname{curl}_{\varepsilon} \wedge g \cdot \omega
$$

we have

$$
\left|-\int v\left(\partial_{t} f+v \cdot \nabla_{x} f\right) \mathrm{d} c+F_{\varepsilon, \delta}\right| \leq C_{f}(\varepsilon+\delta)
$$


From every subsequence of $F_{\varepsilon, \delta}$, we can extract a sequence that converges to

$$
F=-\int v\left(\partial_{t} f+v \cdot \nabla_{x} f\right) \mathrm{d} c
$$

which does not depend on the subsequence.

Using the uniqueness of the limit, we see that the whole sequence $F_{\varepsilon, \delta}$ converges. Using again the uniqueness of the limit, we deduce that there exists $H$ (limit of a subsequence $H_{\varepsilon}$ ) so that

$$
\partial_{t} v+c v \cdot \nabla_{x} v-\underline{c} \partial_{t} H+c v \wedge \operatorname{curl} H=0
$$

which completes the proofs.

\section{REFERENCES}

[1] F. Barthe, Optimal Young's inequality and its converse: A simple proof. Geom. Funct. Anal. 8 (1998) 234-242.

[2] J.-D. Benamou and Y. Brenier, A computational fluid mechanics solution to the Monge-Kantorovich mass transfer problem. Numer. Math. 84 (2000) 375-393.

[3] M. Born and L. Infeld, Foundations of the new field theory. Proc. Roy. Soc. London A 144 (1934) 425-451.

[4] G. Bouchitté and G. Buttazzo, Characterization of optimal shapes and masses through Monge-Kantorovich equation. J. Eur. Math. Soc. (JEMS) 3 (2001) 139-168.

[5] Y. Brenier, A combinatorial algorithm for the Euler equations of incompressible flows, in Proc. of the Eighth International Conference on Computing Methods in Applied Sciences and Engineering. Versailles (1987). Comput. Methods Appl. Mech. Engrg. 75 (1989) 325-332.

[6] Y. Brenier, Décomposition polaire et réarrangement monotone des champs de vecteurs. C. R. Acad. Sci. Paris Sér. I Math. 305 (1987) 805-808.

[7] Y. Brenier, Polar factorization and monotone rearrangement of vector-valued functions. Comm. Pure Appl. Math. 64 (1991) 375-417.

[8] Y. Brenier, A homogenized model for vortex sheets. Arch. Rational Mech. Anal. 138 (1997) 319-353.

[9] Y. Brenier, Minimal geodesics on groups of volume-preserving maps and generalized solutions of the Euler equations. Comm. Pure Appl. Math. 52 (1999) 411-452.

[10] Y. Brenier, Extension of the Monge-Kantorovich theory to classical electrodynamics. Summer School on mass transportation methods in kinetic theory and hydrodynamics. Ponta Delgada, Azores, Portugal (2000).

[11] H. Brézis, Analyse fonctionnelle. Masson, Paris (1974).

[12] L.A. Caffarelli, Boundary regularity of maps with convex potentials. Ann. of Math. (2) 144 (1996) 453-496.

[13] M.J. Cullen and R.J. Purser, An extended Lagrangian theory of semigeostrophic frontogenesis. J. Atmos. Sci. 41 (1984) 1477-1497.

[14] L.C. Evans and W. Gangbo, Differential equations methods for the Monge-Kantorovich mass transfer problem. Mem. Amer. Math. Soc. 137 (1999).

[15] W. Gangbo and R.J. McCann, The geometry of optimal transportation. Acta Math. 177 (1996) 113-161.

[16] D. Kinderlehrer, R. Jordan and F. Otto, The variational formulation of the Fokker-Planck equation. SIAM J. Math. Anal. 29 (1998) 1-17.

[17] L.V. Kantorovich, On a problem of Monge. Uspekhi Mat. Nauk. 3 (1948) 225-226.

[18] R.J. McCann, A convexity principle for interacting gases. Adv. Math. 128 (1997) 153-179.

[19] F. Otto, Viscous fingering: An optimal bound on the growth rate of the mixing zone. SIAM J. Appl. Math. 57 (1997) $982-990$.

[20] F. Otto, The geometry of dissipative evolution equations: The porous medium equation. Comm. Partial Differential Equations 26 (2001) 101-174

[21] F. Otto and C. Villani, Generalization of an inequality by Talagrand and links with the logarithmic Sobolev inequality. $J$. Funct. Anal. 173 (2000) 361-400.

[22] A.V. Pogorelov, The Minkowski multidimensional problem. John Wiley, New York-Toronto-London, Scr. Ser. in Math. (1978).

[23] S.T. Rachev and L. Rüschendorf, Mass transportation problems, Vols. I and II. Probability and its Applications. SpringerVerlag.

[24] G. Strang, Introduction to applied mathematics. Wellesley-Cambridge Press, Wellesley, MA (1986).

[25] V.N. Sudakov, Geometric problems in the theory of infinite-dimensional probability distributions. Proc. Steklov Inst. 141 (1979) 1-178. 